\title{
REGULARITY PROPERTIES FOR TRIPLE SYSTEMS
}

\author{
BRENDAN NAGLE AND VOJTĚCH RÖDL
}

\begin{abstract}
Szemerédi's Regularity Lemma proved to be a powerful tool in the area of extremal graph theory [5]. Many of its applications are based on the following technical fact: If $G$ is a $k$-partite graph with $V(G)=\bigcup_{i=1}^{k} V_{i},\left|V_{i}\right|=n$ for all $i \in[k]$, and all pairs $\left\{V_{i}, V_{j}\right\}, 1 \leq i<j \leq k$, are $\epsilon$-regular of density $d$, then $G$ contains $d_{\left(\begin{array}{c}k \\ 2\end{array}\right)} n^{k}(1+f(\epsilon))$ cliques $K_{k}^{(2)}$, where $f(\epsilon) \rightarrow 0$ as $\epsilon \rightarrow 0$.

The aim of this paper is to establish the analogous statement for 3-uniform hypergraphs. Our result, which we refer to as The Counting Lemma, together with Theorem 3.5 of [2], a Regularity Lemma for Hypergraphs, can be applied in various situations as Szemerédi's Regularity Lemma is for graphs. Some of these applications are discussed in the papers [3], [4] and [7], as well as in upcoming papers of the authors and others.
\end{abstract}

\section{Contents}

1. Introduction

1.1. Szemerédi's Regularity Lemma 2

1.2. This Paper and its Itinerary 4

1.3. Why the Hypergraph Regularity Lemma of [2] is technical 4

Acknowledgement 5

2. Definitions, Notation, and Auxiliary Facts 5

2.1. Graphs and Cylinders 5

2.2. 3-Uniform Hypergraphs and 3-cylinders 7

3. The Counting Lemma 9

3.1. The Statement of the Counting Lemma 9

3.2. Strategy for Proving the Counting Lemma 10

3.3. Proof of Claim 3.2.3 12

4. Background Material for the Proof of $R(k-1) \Rightarrow D(k) \quad 14$

4.1. Basic Facts 14

4.2. Regular Couples $\quad 15$

4.3. Link Graphs 16

5. $R(k-1) \Rightarrow D(k) \quad 17$

5.1. Lemma 5.1.1 and $R(k-1) \Rightarrow D(k) \quad 17$

5.2. Proof of Claim 5.1.2 18

5.3. Proof of Lemma 5.1.1 19

6. The Regularity Lemma 28

6.1. Definitions for the Regularity Lemma 28

6.2. Statement of the Regularity Lemma 30

7. $D(k) \Longrightarrow R(k) \quad 30$ 
7.1. Lemma 7.1.1 31

7.2. $\quad$ Proving Lemma 7.1.1 32

7.3. Itinerary for the Proof of Proposition 7.2.4 36

7.4. The Proof of Proposition 7.2.4 43

7.5. Proof of Lemma 7.3.14 46

8. The Upper Bound of Theorem 3.1.1 55

8.1. Review of Theorem 3.1.1

8.2. Proof of Proposition 8.1.3 56

9. Concluding Remarks $\quad 59$

Index $\quad 61$

$\begin{array}{ll}\text { References } & 63\end{array}$

\section{INTRODUCTION}

Extremal set theory is a well studied and broad subject within the field of combinatorial mathematics. Problems from this discipline, in the most general sense, are concerned with the determination of thresholds on quantitative properties one set system should have in order to contain another set system as a subsystem.

While containment problems are often difficult in a deterministic setting, one can consider the same problems in a random setting, often with some advantages. Here, we consider a random set system as a hypergraph whose edges were randomly generated from a base set. One nice feature of a random set system is that as a consequence of the edges being randomly generated, one can expect a fairly even distribution of the edges. With an even distribution of the edges of a random set system likely, one is afforded tools for trying to determine if the random set system contains a given subsystem. As a drawback, however, a random set system is not a concrete set system, but rather a probability space. Hence, all conclusions made on the containment of a given subsystem in our random set system can only be in terms of certain probabilities.

In the deterministic setting, one has the possibility of conclusively deciding whether or not a concrete set system contains a given subsystem. However, one is not guaranteed the even distribution of edges that in the random setting played a helpful role in deciding containment. If the set systems under consideration are graphs, one successful approach for the problem of graph containment in the deterministic setting has been the use of a powerful lemma of Szemerédi which in some sense combines the deterministic and random settings.

\subsection{Szemerédi's Regularity Lemma.}

In the course of proving his celebrated Density Theorem, E. Szemerédi established a lemma which decomposes the edge set of any graph into "random like pieces" (cf., [12], [5]). He later established a more applicable version, the well known Regularity Lemma, in [11]. We give a precise account in what follows.

For a graph $G=(V, E)$ and two disjoint sets $A, B \subset V$, we denote by $E(A, B)$ the set of edges $\{a, b\} \in G$ with $a \in A$ and $b \in B$ and put $e(A, B)=|E(A, B)|$. 
We also set $d(A, B)=d\left(G_{A B}\right)=e(A, B) /|A||B|$ for the density of the bipartite graph $G_{A B}=(A \cup B, E(A, B))$.

Let $\epsilon>0$ be given. We say that a pair $A, B$ is $\epsilon$-regular if $\left|d(A, B)-d\left(A^{\prime}, B^{\prime}\right)\right|<\epsilon$ holds whenever $A^{\prime} \subset A, B^{\prime} \subset B$, and $\left|A^{\prime}\right|>\epsilon|A|,\left|B^{\prime}\right|>\epsilon|B|$. We call a partition $V=V_{0} \cup V_{1} \cup \cdots \cup V_{t}$ an equitable partition if it satisfies $\left|V_{1}\right|=\left|V_{2}\right|=\cdots=\left|V_{t}\right|$ and $\left|V_{0}\right|<t$; we call an equitable partition $\epsilon$-regular if all but $\epsilon\left(\begin{array}{c}t \\ 2\end{array}\right)$ pairs $V_{i}, V_{j}$ are $\epsilon$-regular.

Theorem 1.1.1. Szemerédi's Regularity Lemma. Let $\epsilon>0$ be given and let $k$ be a positive integer. There exist positive integers $N=N(\epsilon, k)$ and $K=K(\epsilon, k)$ such that any graph $G=(V, E)$ with $|V|=n \geq N$ vertices admits an $\epsilon$-regular equitable partition $V=V_{0} \cup V_{1} \cup \cdots \cup V_{t}$ with $t$ satisfying $k \leq t \leq K$.

Szemerédi's Lemma is a powerful tool in the area of extremal graph theory. One of its most important consequences is that it can help decide if a graph contains a fixed subgraph. Suppose that a (large) graph $G$ is given along with an $\epsilon$-regular partition $V=V_{0} \cup V_{1} \ldots \cup V_{t}$, and let $H$ be a fixed graph. If enough of the $\epsilon$-regular pairs $\left\{V_{i}, V_{j}\right\}$ are dense enough with respect to $\epsilon$, we may build a copy of $H$ within this collection of bipartite graphs $E\left(V_{i}, V_{j}\right)$. This observation is due to the following fact which may be appropriately called the Counting Fact for Graphs.

Fact 1.1.2. The Counting Fact for Graphs. Suppose $G$ is a k-partite graph with $V(G)=\bigcup_{i=1}^{k} V_{i},\left|V_{i}\right|=n$ for all $i \in[k]$, and all pairs $\left\{V_{i}, V_{j}\right\}, 1 \leq i<j \leq k$, are $\epsilon$-regular of density $d$. Then $G$ contains $d^{\left(\begin{array}{c}k \\ 2\end{array}\right)} n^{k}(1+f(\epsilon))$ cliques $K_{k}^{(2)}$, where $f(\epsilon) \rightarrow 0$ as $\epsilon \rightarrow 0$.

In the following two theorems, we provide examples of when combining Szemerédi's Regularity Lemma with Fact 1.1.2 was useful in containment problems. (in the proceeding two examples, the reader is not expected to see the connection between the examples and Fact 1.1.2)

One of the first applications of Fact 1.1.2 (applied when $k=3$ ) was the following result of Ruzsa and Szemerédi [10].

Theorem 1.1.3. Ruzsa, Szemerédi (1976). For every $\epsilon>0$, there exists $n_{0}=n_{0}(\epsilon)$ such that if $H_{n}$ is a 3-uniform hypergraph on $n \geq n_{0}$ vertices not containing 6 points with 3 or more triples, then $\left|H_{n}\right|<\epsilon n^{2}$.

In the next example, for a fixed graph $F$, we denote by $F_{n}(F)$ the number of distinct labeled graphs $G$ on $n$ vertices not containing $F$ as a subgraph. We denote by $\operatorname{ex}(n, F)$ the largest number of edges of any graph $G$ on $n$ vertices not containing $F$ as a subgraph.

Theorem 1.1.4. Erdős, Frankl, Rödl (1986). If $F$ is any graph with $\chi(F)>2$, then

$$
F_{n}(F)=2^{\operatorname{ex}(n, F)(1+o(1))}
$$

where $o(1) \rightarrow 0$ as $n \rightarrow \infty$.

For more on Theorem 1.1.4, see [1]. 


\subsection{This Paper and its Itinerary.}

The aim of this paper is to establish a similar technique for 3-uniform hypergraphs. More specifically, using the Hypergraph Regularity Lemma from [2] for 3-uniform hypergraphs, one is guaranteed a decomposition of the triples of a hypergraph $\mathcal{H}$ analogous to the decomposition of pairs in Szemerédi's Regularity Lemma for graphs (cf., Theorem 1.1.1). Within the partition obtained from The Hypergraph Regularity Lemma, if there is a sufficient number of "dense and regular" pieces, we show a statement analogous to Fact 1.1.2, that the hypergraph $\mathcal{H}$ contains a specific number of cliques $K_{k}^{(3)}$ in those pieces. We refer to our main theorem as The Counting Lemma.

In Theorem 3.1.1 of Section 3, we provide a statement for 3-uniform hypergraphs analogous to Fact 1.1.2. We refer to Theorem 3.1.1 as The Counting Lemma. Since its statement is quite complicated, we defer all discussion of it until Section 3, by which time all relevant concepts are defined. In Section 2, we give an exposition of background concepts needed for the remainder of this paper. Over Sections 4-8, we give the proof of Theorem 3.1.1. Note that in Section 6, we needed to develop a regularity lemma, Theorem 6.2.1, which naturally extends Theorem 3.4 of [2].

\subsection{Why the Hypergraph Regularity Lemma of [2] is technical.}

One can conclude that the Hypergraph Regularity Lemma must be structurally more complicated than that in Theorem 1.1.1. Indeed, for a hypergraph $\mathcal{H}=(V, E)$ and three disjoint sets $A, B, C \subset V$, we denote by $E(A, B, C)$ the set of triples $\{a, b, c\} \in \mathcal{H}$ with $a \in A, b \in B$, and $c \in C$, and put $e(A, B, C)=|E(A, B, C)|$. We also set $d(A, B, C)=d\left(\mathcal{H}_{A B C}\right)=e(A, B, C) /|A||B||C|$ for the density of the hypergraph $\mathcal{H}_{A B C}=$ $(A \cup B \cup C, E(A, B, C))$. For a given $\epsilon>0$, we say the triple $A, B, C$ is $\epsilon$-regular if for any $A^{\prime}, B^{\prime}, C^{\prime}, A^{\prime} \subset A, B^{\prime} \subset B, C^{\prime} \subset C,\left|A^{\prime}\right|>\epsilon|A|,\left|B^{\prime}\right|>\epsilon|B|,\left|C^{\prime}\right|>\epsilon|C|$,

$$
\left|d\left(A^{\prime}, B^{\prime}, C^{\prime}\right)-d(A, B, C)\right|<\epsilon \text {. }
$$

Following the original proof of Theorem 1.1.1, one can prove a statement for hypergraphs analogous to Theorem 1.1.1. That is, one can prove a statement establishing that every 3-uniform hypergraph (in fact, $r$-uniform hypergraph) admits an equitable partition $V=$ $V_{0} \cup V_{1} \cup \ldots \cup V_{t}$ with all but $\epsilon\left(\begin{array}{l}t \\ 3\end{array}\right)$ triples $V_{i}, V_{j}, V_{k} \epsilon$-regular, $1 \leq i<j<k \leq t$. However, no statement like Fact 1.1.2 can be obtained, as the following example illustrates.

Example 1.3.1. For every $\epsilon>0$, there exists a 3-uniform 4-partite hypergraph $\mathcal{H}$ with partite sets $V_{1}, V_{2}, V_{3}, V_{4}$, such that every triple of partite sets $V_{i}, V_{j}, V_{k}, 1 \leq i<j<$ $k \leq 4$, is both dense and $\epsilon$-regular, but still $\mathcal{H}$ contains no copies of the clique $K_{4}^{(3)}$.

Indeed, let $\epsilon>0$ be given. Suppose $V_{1}, V_{2}, V_{3}, V_{4}$ are four pairwise disjoint sets, each of cardinality $n$. We construct a random 3-uniform 4-partite hypergraph $\mathcal{H}$ with vertex set $V=V(\mathcal{H})=V_{1} \cup V_{2} \cup V_{3} \cup V_{4}$ and edge set given as follows: For all pairs $i, j, 1 \leq i<j \leq 4$, consider the following random coloring of the edges $K\left(V_{i}, V_{j}\right)=$ $\left\{\left\{v_{i}, v_{j}\right\}: v_{i} \in V_{i}, v_{j} \in V_{j}\right\}$ : for each edge $\left\{v_{i}, v_{j}\right\} \in K\left(V_{i}, V_{j}\right)$, independently and uniformly at random color the edge $\left\{v_{i}, v_{j}\right\}$ red with probability $\frac{1}{2}$ or blue with probability $\frac{1}{2}$. Then for $1 \leq i<j<k \leq 4, v_{i} \in V_{i}, v_{j} \in V_{j}, v_{k} \in V_{k},\left\{v_{i}, v_{j}, v_{k}\right\} \in \mathcal{H}$ if and only if edges $\left\{v_{i}, v_{j}\right\}$ and $\left\{v_{i}, v_{k}\right\}$ receive different colors. One can show that with probability tending to 1 with $n$ tending to $\infty, \mathcal{H}$ satisfies the following two properties: 
1. For any $i, j, k, 1 \leq i<j<k \leq 4$,

$$
\left|d\left(V_{i}, V_{j}, V_{k}\right)-\frac{1}{2}\right|<\epsilon
$$

2. All triples $V_{i}, V_{j}, V_{k}$ are $\epsilon$-regular, $1 \leq i<j<k \leq 4$.

However, it follows by the construction above that $\mathcal{H}$ contains no copies of $K_{4}^{(3)}$. Indeed, assume $\left\{v_{1}, v_{2}, v_{3}, v_{4}\right\}$ spans a copy of $K_{4}^{(3)}$ in $\mathcal{H}$, where for each $i, 1 \leq i \leq 4, v_{i} \in V_{i}$. It follows by the Pigeon Hole Principle that there exist $j, k, 2 \leq j<k \leq 4$, such that edges $\left\{v_{1}, v_{j}\right\}$ and $\left\{v_{1}, v_{k}\right\}$ are colored by the same color. This is, however, a contradiction.

Acknowledgement. We thank Dwight Duffus and Ronald Gould for many helpful comments which improved this manuscript. We also thank Yulia Dementieva for her helpful comments with certain sections of this manuscript.

\section{Definitions, Notation, and Auxiliary Facts}

We begin this section by providing some background material. We start by giving some very basic definitions and notation which appear pervasively throughout the paper.

For a natural number $n$, we use the notation $[n]$ to denote the set $\{1, \ldots, n\}$. If $X$ is any finite set and $l$ is any natural number so that $l \leq|X|$, we denote by $[X]^{l}$ the set $\{L \subseteq X:|L|=l\}$. For simplicity, $[n]^{l}$ denotes $[[n]]^{l}$. Let $X$ and $Y$ be two sets, $X \cap Y=\emptyset$. We abuse the cartesian product notation $\times$ by defining $X \times Y=\{\{x, y\}: x \in X, y \in Y\}$.

\subsection{Graphs and Cylinders.}

In this subsection, we provide definitions and notation pertaining to graphs. A graph $G$ on a finite vertex set $V=\left\{v_{1}, \ldots, v_{n}\right\}$ is defined as a family $G \subseteq[V]^{2}$. If $G=[V]^{2}$, we call $G$ a clique of size $n$, and in particular, if $n=3$, we call $G$ a triangle. When we do not specify a vertex set, we denote a clique of size $n$ by $K_{n}^{(2)}$.

Let $k$ be a natural number, $k \leq n$. We often specify that the graph $G$ is $k$-partite with $k$-partition $\left(V_{1}, \ldots, V_{k}\right)$. As usual, this means that there exists a partition of the vertex set $V$ of $G, V=V_{1} \cup \ldots \cup V_{k}$, where for any $i \in[k], G \cap\left[V_{i}\right]^{2}=\emptyset$. Often, we consider special bipartite (that is, 2-partite) subgraphs of a graph $G$. For nonempty disjoint subsets $A \subseteq$ $V(G)$ and $B \subseteq V(G)$, we denote by $G[A, B]$ that subgraph $\{\{a, b\} \in G: a \in A, b \in B\}$.

Definition 2.1.1. We refer to any $k$-partite graph $G$ with $k$-partition $\left(V_{1}, \ldots, V_{k}\right)$ as a $k$ partite cylinder, and write $G$ as $G=\bigcup_{1 \leq i<j \leq k} G^{i j}$ where $G^{i j}=G\left[V_{i}, V_{j}\right]=\left\{\left\{v_{i}, v_{j}\right\} \in G\right.$ : $\left.v_{i} \in V_{i}, v_{j} \in V_{j}\right\}$. If $B \subseteq[k],|B|=b$, then the $b$-partite cylinder $G(B)=\bigcup_{\{i, j\} \in[B]^{2}} G^{i j}$ is referred to as the $B$-cylinder of $G$, and if $b=3$, the $B$-cylinder $G(B)$ is referred to as a triad. Note that if $B=[k], G(B)=G$.

We note that a cylinder is a graph whose vertex set is given with a fixed $k$-partition.

Definition 2.1.2. Suppose $G \subseteq[V]^{2}$ is a graph with vertex set $V=V(G)$, and let $X, Y \subseteq V$ be two nonempty disjoint subsets of $V$. We define the density of the pair $X, Y$ with respect to $G$, denoted $d_{G}(X, Y)$, as 


$$
d_{G}(X, Y)=\frac{|G[X, Y]|}{|X||Y|}
$$

Definition 2.1.3. Suppose $G \subseteq[V]^{2}$ is a graph with vertex set $V=V(G)$, and let $\epsilon>0$ be given. Let $X, Y \subseteq V$ be two nonempty disjoint subsets of $V$. We say that the pair $X, Y$ is $\epsilon$-regular if whenever $X^{\prime} \subseteq X,\left|X^{\prime}\right|>\epsilon|X|$ and $Y^{\prime} \subseteq Y,\left|Y^{\prime}\right|>\epsilon|Y|$, then

$$
\left|d_{G}\left(X^{\prime}, Y^{\prime}\right)-d_{G}(X, Y)\right|<\epsilon .
$$

We use the following slight alteration of the concept of $\epsilon$-regularity. For positive $\lambda$ and $\epsilon$ given, we say that the graph $G[X, Y]$ is a $(\lambda, \epsilon, 2)$-cylinder provided that whenever $X^{\prime} \subseteq X,\left|X^{\prime}\right|>\epsilon|X|$ and $Y^{\prime} \subseteq Y,\left|Y^{\prime}\right|>\epsilon|Y|$, then

$$
\frac{1}{\lambda}(1-\epsilon)<d_{G}\left(X^{\prime}, Y^{\prime}\right)<\frac{1}{\lambda}(1+\epsilon) .
$$

More generally, we give the following definition.

Definition 2.1.4. Suppose $G=\bigcup_{1 \leq i<j \leq k} G^{i j}$ is a $k$-partite cylinder with $k$-partition $\left(V_{1}, \ldots, V_{k}\right)$ and let $\lambda>0, \epsilon>0$ be given. We call $G$ a $(\lambda, \epsilon, k)$-cylinder provided all pairs $V_{i}, V_{j}, 1 \leq i<j \leq k$, induce $G^{i j}$ satisfying that whenever $V_{i}^{\prime} \subseteq V_{i},\left|V_{i}^{\prime}\right|>\epsilon\left|V_{i}\right|$, and $V_{j}^{\prime} \subseteq V_{j},\left|V_{j}^{\prime}\right|>\epsilon\left|V_{j}\right|$ then

$$
\frac{1}{\lambda}(1-\epsilon)<d_{G^{i j}}\left(V_{i}^{\prime}, V_{j}^{\prime}\right)<\frac{1}{\lambda}(1+\epsilon) .
$$

In other words, each $G^{i j}$ is a $(\lambda, \epsilon, 2)$-cylinder. Note that for $B \subseteq[k],|B|=b$, the $B$-cylinder $G(B)=\bigcup_{\{i, j\} \in[B]^{2}} G^{i j}$ is a $(\lambda, \epsilon, b)$-cylinder whenever $G=\bigcup_{1 \leq i<j \leq k} G^{i j}$ is a $(\lambda, \epsilon, k)$-cylinder. We refer to the $B$-cylinder $G(B)$ as the $(\lambda, \epsilon, B)$-cylinder of $G$.

We now compare the two notions of regularity above. A $(\lambda, \epsilon, 2)$-cylinder $G^{i j}$ is $\epsilon$ regular with density $d=d_{G^{i j}}\left(V_{i}, V_{j}\right)$ satisfying

$$
\left|d-\frac{1}{\lambda}\right|<\frac{\epsilon}{\lambda}
$$

On the other hand, in a graph $G$, an $\epsilon$-regular pair $X, Y$ with density $d_{G}(X, Y)$ satisfying $\frac{1}{\lambda}-\epsilon<d_{G}(X, Y)<\frac{1}{\lambda}+\epsilon$ is a $(\lambda, 2 \epsilon \lambda, 2)$-cylinder.

Suppose $G$ is a $k$-partite cylinder with $k$-partition $\left(V_{1}, \ldots, V_{k}\right)$. For a vertex $v \in V$, and $j \in[k]$, we denote by $N_{j}(v)$ the $j$-neighborhood of $v$, that is, $N_{j}(v)=\left\{w \in V_{j}:\{v, w\} \in G\right\}$. We now state the following simple fact about $(\lambda, \epsilon, k)$-cylinders concerning neighborhood sizes.

Fact 2.1.5. Suppose $G=\bigcup_{1 \leq i<j \leq k} G^{i j}$ is a $(\lambda, \epsilon, k)$-cylinder with $k$-partition $\left(V_{1}, \ldots, V_{k}\right)$, $\left|V_{1}\right|=\ldots=\left|V_{k}\right|=m$. Fix $i \in[k]$. All but $2 k \epsilon m$ vertices $v_{i} \in V_{i}$ satisfy that for all $j \in[k], j \neq i$,

$$
\frac{m}{\lambda}(1-\epsilon)<\left|N_{j}\left(v_{i}\right)\right|<\frac{m}{\lambda}(1+\epsilon) .
$$


For future reference, we also make the following remarks. Sometimes we consider the situation when two $k$-partite cylinders $G$ and $F$ are simultaneously defined on the same vertex set $V$ with $k$-partition $\left(V_{1}, \ldots, V_{k}\right)$. In such situations, we still want to denote the $j$-neighbors in each cylinder. We denote by $N_{G, j}(v)$ the $j$-neighborhood of the vertex $v$ in the cylinder $G$, that is, $N_{G, j}(v)=\left\{w \in V_{j}:\{v, w\} \in G\right\}$. Similarly, $N_{F, j}(v)=\left\{w \in V_{j}:\{v, w\} \in F\right\}$.

We now define an auxiliary set system pertaining to a $k$-partite cylinder $G$.

Definition 2.1.6. For a $k$-partite cylinder $G$, we denote by $\mathcal{K}_{j}^{(2)}(G), 1 \leq j \leq k$, that $j$-uniform hypergraph whose edges are precisely those $j$-element subsets of $V(G)$ which span cliques of order $j$ in $G$. Note that the quantity $\left|\mathcal{K}_{j}^{(2)}(G)\right|$ counts the total number of cliques in $G$ of order $j$, that is, $\left|\mathcal{K}_{j}^{(2)}(G)\right|=\left|\left\{X \subseteq V(G):|X|=j,[X]^{2} \subseteq G\right\}\right|$.

For a $(\lambda, \epsilon, k)$-cylinder $G$, the quantity $\left|\mathcal{K}_{j}(G)\right|$ is easy to estimate, as the following fact shows.

Fact 2.1.7. For any positive integers $k, \lambda$, and suitably small positive reals $\epsilon$, there exists a function $\theta_{k, \lambda}(\epsilon), \theta_{k, \lambda}(\epsilon) \rightarrow 0$ as $\epsilon \rightarrow 0$, such that whenever $G$ is a $(\lambda, \epsilon, k)$-cylinder with $k$-partition $\left(V_{1}, \ldots, V_{k}\right),\left|V_{1}\right|=\ldots=\left|V_{k}\right|=m$, then

$$
\left(1-\theta_{k, \lambda}(\epsilon)\right) \frac{m^{k}}{\lambda^{\left(\begin{array}{c}
k \\
2
\end{array}\right)}}<\left|\mathcal{K}_{k}^{(2)}(G)\right|<\left(1+\theta_{k, \lambda}(\epsilon)\right) \frac{m^{k}}{\lambda^{\left(\begin{array}{c}
k \\
2
\end{array}\right)}} .
$$

The proof of Fact 2.1.7 is an easy exercise.

\subsection{3-Uniform Hypergraphs and 3-cylinders.}

In this subsection, we provide definitions and notation concerning 3-uniform hypergraphs. A 3-uniform hypergraph $\mathcal{H}$, otherwise called a triple system, on a finite vertex set $V=\left\{v_{1}, \ldots, v_{n}\right\}$ is defined as a family $\mathcal{H} \subseteq[V]^{3}$. If $\mathcal{H}=[V]^{3}$, we call $\mathcal{H}$ a clique of size $n$. Similar to graphs, when we don't specify a vertex set, we denote a hypergraph clique of size $n$ by $K_{n}^{(3)}$.

Let $k$ be a natural number, $k \leq n$. As in the case of graphs, we often specify that the hypergraph $\mathcal{H}$ is $k$-partite with given $k$-partition $\left(V_{1}, \ldots, V_{k}\right)$. Again, this means that there exists a partition of the vertex set $V$ of $\mathcal{H}, V=V_{1} \cup \ldots \cup V_{k}$, where for any pair $\{i, j\} \in[k]^{2}, \mathcal{H} \cap\left[V_{i} \cup V_{j}\right]^{3}=\emptyset$.

Definition 2.2.1. We refer to any $k$-partite, 3 -uniform hypergraph $\mathcal{H}$ with $k$-partition $\left(V_{1}, \ldots, V_{k}\right)$ as a $k$-partite 3 -cylinder. For $B \subseteq[k]$, we define the $B$-3-cylinder of $\mathcal{H}$ as that subhypergraph $\mathcal{H}(B)$ of $\mathcal{H}$ induced on $\bigcup_{i \in B} V_{i}$. Note that if $B=[k], \mathcal{H}(B)=\mathcal{H}$.

Definition 2.2.2. Suppose that $G$ is a $k$-partite cylinder given with $k$-partition $\left(V_{1}, \ldots, V_{k}\right)$, and $\mathcal{H}$ is a $k$-partite 3 -cylinder. We say that $G$ underlies the 3 -cylinder $\mathcal{H}$ if $\mathcal{H} \subseteq \mathcal{K}_{3}^{(2)}(G)$.

As in Definition 2.1.6, we define an auxiliary set system pertaining to the 3 -cylinder $\mathcal{H}$.

Definition 2.2.3. If $\mathcal{H}$ is a $k$-partite 3 -cylinder, then for $1 \leq j \leq k, \mathcal{K}_{j}^{(3)}(\mathcal{H})$ denotes that $j$-uniform hypergraph whose edges are precisely those $j$-element subsets of $V(\mathcal{H})$ which 
span a clique of order $j$ in $\mathcal{H}$. Note that the quantity $\left|\mathcal{K}_{j}^{(3)}(\mathcal{H})\right|$ counts the total number of cliques in $\mathcal{H}$ of order $j$, that is, $\left|\mathcal{K}_{j}^{(3)}(\mathcal{H})\right|=\left|\left\{X \subseteq V(\mathcal{H}):|X|=j,[X]^{3} \subseteq \mathcal{H}\right\}\right|$.

Definition 2.2.4. Let $\mathcal{H}$ be a $k$-partite 3 -cylinder with underlying $k$-partite cylinder $G=\bigcup_{1 \leq i<j \leq k} G^{i j}$, and let $B \subseteq[k],|B|=b$. For the $B$-cylinder $G(B)$, we define the density $\bar{d}_{\mathcal{H}}(\bar{G}(B))$ of $\mathcal{H}$ with respect to the $B$-cylinder $G(B)$ as

$$
d_{\mathcal{H}}(G(B))= \begin{cases}\frac{\left|\mathcal{K}_{b}^{(2)}(G(B)) \cap \mathcal{K}_{b}^{(3)}(\mathcal{H}(B))\right|}{\left|\mathcal{K}_{b}^{(2)}(G(B))\right|} & \text { if }\left|\mathcal{K}_{b}^{(2)}(G(B))\right|>0 \\ 0 & \text { otherwise }\end{cases}
$$

In other words, the density counts the proportion of copies of $K_{b}^{(2)}$ in $G(B)$ which underlie copies of $K_{b}^{(3)}$ in $\mathcal{H}(B)$.

More generally, let $Q \subseteq G(B), B \subseteq[k],|B|=b$, where $Q=\bigcup_{\{i, j\} \in[B]^{2}} Q^{i j}$. One can define the density $d_{\mathcal{H}}(Q)$ of $\mathcal{H}$ with respect to $Q$ as

$$
d_{\mathcal{H}}(Q)= \begin{cases}\frac{\left|\mathcal{K}_{b}^{(3)}(\mathcal{H}) \cap \mathcal{K}_{b}^{(2)}(Q)\right|}{\left|\mathcal{K}_{b}^{(2)}(Q)\right|} & \text { if }\left|\mathcal{K}_{b}^{(2)}(Q)\right|>0 \\ 0 & \text { otherwise. }\end{cases}
$$

For our purposes, we need an extension of the definition above, and consider a simultaneous density of $\mathcal{H}$ with respect to a fixed $r$-tuple of $b$-partite cylinders $(Q(1), \ldots, Q(r))$.

Definition 2.2.5. Let $\mathcal{H}$ be a $k$-partite 3 -cylinder with underlying $k$-partite cylinder $G=\bigcup_{1 \leq i<j \leq k} G^{i j}$, and let $B \subset[k],|B|=b$. Let $\vec{Q}=\vec{Q}_{B}=(Q(1), \ldots, Q(r))$ be an $r$ tuple of $B$-cylinders $Q(s)=\bigcup_{\{i, j\} \in[B]^{2}} Q^{i j}(s)$ satisfying that for every $s \in[r],\{i, j\} \in[B]^{2}$, $Q^{i j}(s) \subseteq G^{i j}$. We define the density $d_{\mathcal{H}}(\vec{Q})$ of $\vec{Q}$ as

$$
d_{\mathcal{H}}(\vec{Q})= \begin{cases}\frac{\left|\mathcal{K}_{b}^{(3)}(\mathcal{H}) \cap \bigcup_{s=1}^{r} \mathcal{K}_{b}^{(2)}(Q(s))\right|}{\left|\bigcup_{s=1}^{r} \mathcal{K}_{b}^{(2)}(Q(s))\right|} & \text { if }\left|\bigcup_{s=1}^{r} \mathcal{K}_{b}^{(2)}(Q(s))\right|>0 \\ 0 & \text { otherwise. }\end{cases}
$$

We now give a definition which provides a notion of regularity for 3-cylinders.

Definition 2.2.6. Let $\mathcal{H}$ be a $k$-partite 3 -cylinder with underlying cylinder $G=\bigcup_{1 \leq i<j \leq k} G^{i j}$. Let $B \subseteq[k],|B|=b, r$ and $\delta>0$ be given. We say that the $B$-3-cylinder $\mathcal{H}(B)$ is $(\delta, r)$ regular with respect to $G(B)$ if the following regularity condition is satisfied: suppose $\vec{Q}=\vec{Q}_{B}=(Q(1), \ldots, Q(r))$ is an $r$-tuple of $B$-cylinders $Q(s)=\bigcup_{\{i, j\} \in[B]^{2}} Q^{i j}(s)$ satisfying that for all $s \in[r]$, and all $\{i, j\} \in[B]^{2}, Q^{i j}(s) \subseteq G^{i j}$. Then $\left|\bigcup_{s=1}^{r} \mathcal{K}_{b}^{(2)}(Q(s))\right|>$ $\delta\left|\mathcal{K}_{b}^{(2)}(G(B))\right|$ implies

$$
d_{\mathcal{H}}(\vec{Q})>d_{\mathcal{H}}(G(B))-\delta .
$$

If, moreover, it is specified that $\mathcal{H}(B)$ is $(\delta, r)$-regular with respect to $G(B)$ with density $d_{\mathcal{H}}(G(B)) \geq \alpha-\delta$ for some $\alpha$, then we say that the $B$-3-cylinder $\mathcal{H}(B)$ is $(\alpha, \delta, r)$-regular with respect to $G(B)$. If the regularity condition fails to be satisfied for any $\alpha$, we say that $\mathcal{H}(B)$ is $(\delta, r)$-irregular with respect to $G(B)$. 
Note that this is a weaker definition of regularity since only a lower bound on the density of $\vec{Q}$ is guaranteed. The more standard notion of $\epsilon$-regularity which replaces (5) with

$$
\left|d_{\mathcal{H}}(\vec{Q})-d_{\mathcal{H}}(G(B))\right|<\delta
$$

and corresponds to Szemerédi's definition of an $\epsilon$-regular pair was introduced in [2]. Since most of this paper deals with this weaker concept, we decided to alter the standard vocabulary. However, we also require the stronger notion, and so conclude this section with this definition.

Definition 2.2.7. Let $\mathcal{H}$ be a 3-partite 3-cylinder with underlying cylinder $G=G^{12} \cup$ $G^{23} \cup G^{13}$. Let $r$ and $\delta>0$ be given. We say that $\mathcal{H}$ is $(\delta, r)$-fully regular with respect to $G$ if the following regularity condition is satisfied: suppose $\vec{Q}=(Q(1), \ldots, Q(r))$ is an $r$-tuple of triads $Q(s)=\bigcup_{\{i, j\} \in[3]^{2}} Q^{i j}(s)$, where for all $s \in[r],\{i, j\} \in[3]^{2}, Q^{i j}(s) \subseteq G^{i j}$. Then $\left|\bigcup_{s=1}^{r} \mathcal{K}_{3}^{(2)}(Q(s))\right|>\delta\left|\mathcal{K}_{3}^{(2)}(G)\right|$ implies

$$
\left|d_{\mathcal{H}}(\vec{Q})-d_{\mathcal{H}}(G)\right|<\delta .
$$

If, moreover, it is specified that $\mathcal{H}$ is $(\delta, r)$-fully regular with respect to $G$ with density $d_{\mathcal{H}}(G) \in(\alpha-\delta, \alpha+\delta)$ for some $\alpha$, then we say that $\mathcal{H}$ is $(\alpha, \delta, r)$-fully regular with respect to $G$.

Note that $(\delta, r)$-full regularity implies $(\delta, r)$-regularity, but not conversely.

\section{The Counting Lemma}

In this section, we present and comment on the main theorem of this work, The Counting Lemma.

\subsection{The Statement of the Counting Lemma.}

Before we state our theorem, we establish an appropriate context. Since we later alter this context, we refer to the following as Setup 1.

\section{Setup 1:}

For a given integer $k \geq 3$, for a given set $\left\{\alpha_{B}: B \in[k]^{3}\right\}$ of positive reals and for given constants $\delta, \lambda, r$ and $\epsilon$, suppose $\mathcal{H}$ is a triple system and $G$ is an underlying graph satisfying the following:

(i) $\mathcal{H}$ is a $k$-partite 3 -cylinder with $k$-partition $\left(V_{1}, \ldots, V_{k}\right),\left|V_{1}\right|=\ldots=\left|V_{k}\right|=m$.

(ii) $G$ is an underlying $(\lambda, \epsilon, k)$-cylinder of $\mathcal{H}$.

(iii) for all $B \in[k]^{3}, \mathcal{H}(B)$ is $\left(\alpha_{B}, \delta, r\right)$-fully regular with respect to the $\operatorname{triad} G(B)$ (cf., Definition 2.2.7).

The aim of this paper is to establish the following theorem.

Theorem 3.1.1. The Counting Lemma. For all integers $k \geq 4$, sets $\left\{\alpha_{B}: B \in[k]^{3}\right\}$ of positive reals and $\beta>0$, there exists a positive constant $\delta$ so that for all integers $\lambda \geq \frac{1}{\delta}$, there exist $r$, $\epsilon$ such that the following holds: with parameters $k,\left\{\alpha_{B}: B \in[k]^{3}\right\}$, 
$\delta, \lambda, r$ and $\epsilon$, suppose $\mathcal{H}$ is a triple system and $G$ is an underlying graph which satisfy the hypothesis of Setup 1. Then

$$
\frac{\prod_{B \in[k]^{3} \alpha_{B}}}{\left.\lambda^{(k} \begin{array}{c}
k \\
2
\end{array}\right)} m^{k}(1-\beta) \leq\left|\mathcal{K}_{k}^{(3)}(\mathcal{H})\right| \leq \frac{\Pi_{B \in[k]^{3} \alpha_{B}}}{\left.\lambda^{(k} \begin{array}{c}
2 \\
2
\end{array}\right)} m^{k}(1+\beta) .
$$

As mentioned in the Introduction, unlike Fact 1.1.2, the proof of Theorem 3.1.1 is complicated. Sections 4-8 are devoted to proving this theorem.

In proving Theorem 3.1.1, we use the following hierarchy governing the sizes of the constants $\min \left\{\alpha_{B}: B \in[k]^{3}\right\}, \delta, \lambda, r, \epsilon, m$ :

$$
\min \left\{\alpha_{B}: B \in[k]^{3}\right\} \gg \delta \geq \frac{1}{\lambda} \gg \frac{1}{r} \gg \epsilon \gg \frac{1}{m} .
$$

In Theorem 3.1.1, we make the assumption that $\delta \geq \frac{1}{\lambda}$, thus providing a well arranged (linearly ordered) hierarchy on the constants. However, for the other extreme that $\delta \ll \frac{1}{\lambda}$, Theorem 3.1.1 remains true and its proof becomes considerably simpler. Unfortunately, The Hypergraph Regularity Lemma of [2], the tool which in application provides an environment satisfying the conditions of Setup 1, does not us allow us to make the assumption that $\delta \ll \frac{1}{\lambda}$. The reason for this unfortunate outcome is similar to why in the context of Theorem 1.1.1 one may assume $\epsilon \geq \frac{1}{t}$; one may simply set $t_{0} \geq \frac{1}{\epsilon}$.

For the remainder of this paper, the reader may assume the constants with which we work always satisfy the hierarchy given in (8). While we carefully specify the sizes of the promised constants $\delta, r$ and $\epsilon$ in all of our proofs, we make no effort to estimate the size of the integer $m$ seen in (8). We state here that in this entire paper, we assume the integer $m$ is large enough for any argument we make, and indeed, the value $\frac{1}{m}$ may be assumed to be "infinitely smaller" than any other constant considered.

\subsection{Strategy for Proving the Counting Lemma.}

We found it advantageous to focus on the lower bound of (7) for most of this paper. To that end, we formulate a theorem which exclusively discusses this lower bound. Before doing so, however, we discuss the weaker environment in which we work.

\section{Setup 2:}

For a given integer $k \geq 3$, for a given set $\left\{\alpha_{B}: B \in[k]^{3}\right\}$ of positive reals and for given constants $\delta, \lambda, r$ and $\epsilon$, suppose $\mathcal{H}$ is a triple system and $G$ is an underlying graph satisfying the following:

(i) $\mathcal{H}$ is a $k$-partite 3 -cylinder with $k$-partition $\left(V_{1}, \ldots, V_{k}\right),\left|V_{1}\right|=\ldots=\left|V_{k}\right|=m$.

(ii) $G$ is an underlying $(\lambda, \epsilon, k)$-cylinder of $\mathcal{H}$.

(iii) For all $B \in[k]^{3}, \mathcal{H}(B)$ is $\left(\alpha_{B}, \delta, r\right)$-regular with respect to the triad $G(B)$ (cf., Definition 2.2.6).

If moreover, the following also holds:

$\left(i i i^{\prime}\right)$. All the constants $\alpha_{B}$ in the set $\left\{\alpha_{B}: B \in[k]^{3}\right\}$ are the same, (i.e. $\alpha_{B}=\alpha$ for all $\left.B \in[k]^{3}\right)$.

then we refer to this special case of Setup 2 as Setup $2^{\prime}$. 
Theorem 3.2.1. For all integers $k \geq 4$, sets $\left\{\alpha_{B}: B \in[k]^{3}\right\}$ of positive reals and $\beta>0$, there exists a positive constant $\delta$ so that for all integers $\lambda \geq \frac{1}{\delta}$, there exist $r$, $\epsilon$ such that the following holds: with parameters $k,\left\{\alpha_{B}: B \in[k]^{3}\right\}, \delta, \lambda, r$ and $\epsilon$, suppose $\mathcal{H}$ is a triple system and $G$ is an underlying graph which satisfy the hypothesis of Setup 2. Then

$$
\left|\mathcal{K}_{k}^{(3)}(\mathcal{H})\right| \geq \frac{\Pi_{B \in[k]^{3} \alpha_{B}}}{\left.\lambda^{(} \begin{array}{c}
k \\
2
\end{array}\right)} m^{k}(1-\beta) .
$$

We show that (perhaps surprisingly), the upper bound in (7) of Theorem 3.1.1 follows from the lower bound in (7) of Theorem 3.1.1. In other words, Theorem 3.2.1 implies Theorem 3.1.1. We show this implication in Section 8.

To prove Theorem 3.2.1, it suffices to prove the following weaker statement $D(k)$, for all integers $k \geq 4$.

Statement 3.2.2 $(D(k))$. For all positive $\alpha$ and positive $\beta$, there exists a positive constant $\delta$ so that for all integers $\lambda \geq \frac{1}{\delta}$, there exist $r, \epsilon$ such that the following holds: with constants $k, \alpha, \delta, \lambda, r$, and $\epsilon$, suppose $\mathcal{H}$ is a $k$-partite 3 -cylinder and $G$ is a $k$-partite cylinder satisfying the hypothesis of Setup $2^{\prime}$. Then

$$
\left|\mathcal{K}_{k}^{(3)}(\mathcal{H})\right| \geq \frac{\alpha^{\left(\begin{array}{c}
k \\
3
\end{array}\right)}}{\lambda^{\left(\begin{array}{c}
k \\
2
\end{array}\right)}} m^{k}(1-\beta) .
$$

At the end of this section we prove the following claim.

Claim 3.2.3. $D(k)$, for all $k \geq 4$, implies Theorem 3.2.1.

Thus, by Claim 3.2.3, to prove Theorem 3.2.1, it is enough to prove $D(k)$ for all $k \geq 4$. The statement $D(k)$ is proved using induction on $k$. In our induction scheme, we need to prove the following stronger statement $R(k)$, where $k \geq 3$.

Statement 3.2.4 $(R(k))$. For all nonnegative $\alpha$ and positive $\delta_{k}$, there exists a positive constant $\delta$ so that for all integers $\lambda \geq \frac{1}{\delta}$, for all integers $r_{k} \geq 1$, there exist $r, \epsilon$ such that the following holds: with constants $k, \alpha, \delta, \lambda, r$, and $\epsilon$, suppose $\mathcal{H}$ is a k-partite 3-cylinder and $G$ is a $k$-partite cylinder satisfying the hypothesis of Setup $\mathscr{L}^{2}$. Then $\mathcal{H}=\mathcal{H}([k])$ is $\left(\alpha\left(\begin{array}{c}k \\ 3\end{array}\right), \delta_{k}, r_{k}\right)$-regular with respect to $G=G([k])$ (cf., Definition 2.2.6).

In other words, the statement $R(k)$ guarantees an "arbitrarily uniform distribution" of the cliques $K_{k}^{(3)}$ provided all $\left(\begin{array}{l}k \\ 3\end{array}\right)$ corresponding triads are sufficiently regular.

We proceed according to the following scheme:

$$
R(3) \Rightarrow D(4) \Rightarrow R(4) \Rightarrow D(5) \Rightarrow \ldots \Rightarrow R(k-1) \Rightarrow D(k) \Rightarrow R(k) \Rightarrow \ldots
$$

The scheme above establishes the validity of the statement $D(k)$, for all $k \geq 4$. We now outline our strategy for proving the induction scheme in (10). Note that the statement $R(3)$ holds by definition. Note also that the first implication, $R(3) \Rightarrow D(4)$, was essentially proved in [2]. We break the inductive step into the following two implications: 
(1) $R(k-1) \Rightarrow D(k)$.

(2) $D(k) \Rightarrow R(k)$.

In Section 5 , we prove the implication $R(k-1) \Rightarrow D(k)$ from implication (1). We note that our proof of the implication $R(k-1) \Rightarrow D(k)$ in Section 5 follows largely from principles we establish in the upcoming Section 4. We show the implication $D(k) \Rightarrow R(k)$ from implication (2) in Section 7. We note that explicit in our proof of $D(k) \Rightarrow R(k)$ is the use of our Regularity Lemma, which we present in the upcoming Section 6.

\subsection{Proof of Claim 3.2.3.}

We conclude this section now with a proof for Claim 3.2.3.

\section{Proof of Claim 3.2.3.}

We begin our proof of Claim 3.2.3 by stating the following fact.

Fact 3.3.1. Let $\alpha, \delta, \epsilon$ be given positive reals with $\delta<\frac{\alpha}{4}$ and let $\lambda$ and $r$ be given positive integers. Let $\alpha^{\prime}$ be a given positive real satisfying $\alpha^{\prime} \leq \alpha$, and let 3-partite triple system $\mathcal{H}$ and underlying graph $G$ satisfy the hypothesis of Setup 2 with constants $k=3, \alpha, \delta$, $\lambda, r$ and $\epsilon$. Then there exists a family

$$
\widetilde{\mathcal{H}}=\left\{\mathcal{H}_{1}, \ldots, \mathcal{H}_{p}\right\}
$$

of 3-partite triple systems with the following properties:

(i) $p=\left\lfloor\frac{\alpha}{\alpha^{\prime}}\right\rfloor$,

(ii) $\mathcal{H}_{i} \cap \mathcal{H}_{j}=\emptyset$ for all $\{i, j\} \in[p]^{2}$, and $\bigcup_{i \in[p]} \mathcal{H}_{i} \subseteq \mathcal{H}$,

(iii) for each $i \in[p], \mathcal{H}_{i}$ is $\left(\alpha^{\prime}, 2 \delta, r\right)$-regular with respect to the underlying $(\lambda, \epsilon, 3)$ cylinder $G$.

The proof of Fact 3.3.1 is found in [6] and [3]. The proof of a similar statement for graphs is given in [2].

The idea of the proof of Claim 3.2.3 is simple. Using Fact 3.3.1, we decompose each of the 3-partite 3-cylinders $\mathcal{H}(B), B \in[k]^{3}$, into roughly $\frac{\alpha_{B}}{\alpha} 3$-partite 3 -cylinders, each of density $\alpha$. For each combination of these sparser "sub-3-cylinders" of $\mathcal{H}(B)$ over all

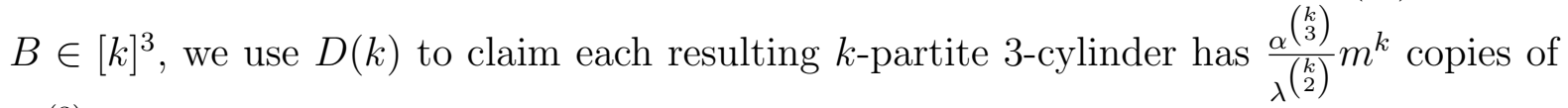
$K_{k}^{(3)}$. Summing over all $\Pi_{B \in[k]^{3} \frac{\alpha_{B}}{\alpha}}$ combinations, we get the required lower bound. We now formally prove Claim 3.2.3.

Let $k \geq 4$ be a given integer, let $\left\{\alpha_{B}: B \in[k]^{3}\right\}$ be a given set of positive reals, and let $\beta>0$ be given. We need to define the promised constant $\delta>0$. Before doing so, we define auxiliary positive constants $\alpha$ and $\beta^{\prime}$ to satisfy

$$
\left(1-\beta^{\prime}\right) \Pi_{B \in[k]^{3}}\left(1-\frac{\alpha}{\alpha_{B}}\right)>1-\beta .
$$

Now, for the constants $k \geq 4, \alpha$ and $\beta^{\prime}$ above, let

$$
\delta=\frac{\delta_{D(k)}\left(k, \alpha, \beta^{\prime}\right)}{2}
$$

where $\delta_{D(k)}\left(k, \alpha, \beta^{\prime}\right)$ is guaranteed to exist by the statement $D(k)$. 
Let $\lambda>\frac{1}{\delta}$ be a given integer. For the constants $k \geq 4, \alpha, \beta^{\prime}, \delta, \lambda$, let

$$
\begin{aligned}
& r=r_{D(k)}\left(k, \alpha, \beta^{\prime}, \delta, \lambda\right), \\
& \epsilon=\epsilon_{D(k)}\left(k, \alpha, \beta^{\prime}, \delta, \lambda\right)
\end{aligned}
$$

be those constants guaranteed to exist by the statement $D(k)$.

With the given parameters $k,\left\{\alpha_{B}: B \in[k]^{3}\right\}, \delta$ from (12), the given integer $\lambda>\frac{1}{\delta}$, and $r$ and $\epsilon$ from (13) and (14) respectively, suppose $\mathcal{H}$ is a $k$-partite triple system and $G$ is an underlying graph of $\mathcal{H}$ satisfying the hypothesis of Setup 2. Our goal is to show

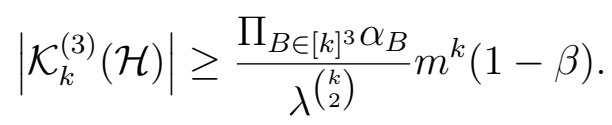

We begin by applying Fact 3.3.1 to each 3-partite 3-cylinder $\mathcal{H}(B)$ with underlying $(\lambda, \epsilon, 3)$-cylinder $G(B), B \in[k]^{3}$. Thus, for each $B \in[k]^{3}$, we obtain a family $\widetilde{\mathcal{H}(B)}=$ $\left\{\mathcal{H}_{i}(B)\right\}, 1 \leq i \leq\left\lfloor\frac{\alpha_{B}}{\alpha}\right\rfloor$ of 3 -partite 3 -cylinders, where:

a. $\mathcal{H}_{i}(B) \cap \mathcal{H}_{j}(B)=\emptyset$, for all $i, j, 1 \leq i<j \leq\left\lfloor\frac{\alpha_{B}}{\alpha}\right\rfloor$, and $\bigcup_{1 \leq i \leq\left\lfloor\frac{\alpha_{B}}{\alpha}\right\rfloor} \mathcal{H}_{i}(B) \subseteq \mathcal{H}(B)$,

b. for each $i, 1 \leq i \leq\left\lfloor\frac{\alpha_{B}}{\alpha}\right\rfloor, \mathcal{H}_{i}(B)$ is $(\alpha, 2 \delta, r)$-regular with respect to the $(\lambda, \epsilon, 3)$ cylinder $G(B)$.

For each of the $\Pi_{B \in[k]^{3}}\left\lfloor\frac{\alpha_{B}}{\alpha}\right\rfloor$ choices of $i_{B}, 1 \leq i_{B} \leq\left\lfloor\frac{\alpha_{B}}{\alpha}\right\rfloor$, consider a vector $\vec{i}=$

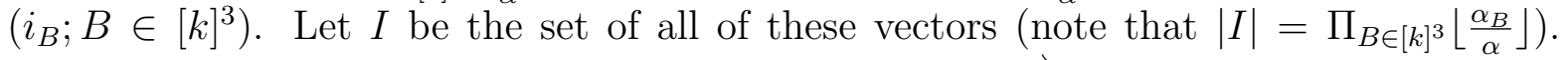
Let $k$-partite 3 -cylinder $\mathcal{H}_{\vec{i}}=\bigcup_{B \in[k]^{3}} \mathcal{H}_{i_{B}}(B)$. For all $\vec{i} \in I, \mathcal{H}_{\vec{i}}$ and $G$ satisfy the conditions of the hypothesis in Setup $2^{\prime}$ with the constants $k, \alpha, 2 \delta, \lambda, r$ and $\epsilon$, where $\delta$ satisfies (12), and $r$ and $\epsilon$ satisfy (13) and (14) respectively. Therefore, we may apply statement $D(k)$ to each $k$-partite 3 -cylinder $\mathcal{H}_{\vec{i}}$ and $G, \vec{i} \in I$, to conclude

$$
\left|\mathcal{K}_{k}^{(3)}\left(\mathcal{H}_{\vec{i}}\right)\right| \geq \frac{\alpha^{\left(\begin{array}{c}
k \\
3
\end{array}\right)}}{\lambda^{\left(\begin{array}{c}
k \\
2
\end{array}\right)}} m^{k}\left(1-\beta^{\prime}\right) .
$$

We therefore conclude

$$
\begin{aligned}
\left|\mathcal{K}_{k}^{(3)}(\mathcal{H})\right| & \geq\left|\bigcup_{\vec{i} \in I} \mathcal{K}_{k}^{(3)}\left(\mathcal{H}_{\vec{i}}\right)\right| \\
& =\sum_{\vec{i} \in I}\left|\mathcal{K}_{k}^{(3)}\left(\mathcal{H}_{\vec{i}}\right)\right|
\end{aligned}
$$

Employing the bound from (16) in (17) yields

$$
\left|\mathcal{K}_{k}^{(3)}(\mathcal{H})\right| \geq \frac{\alpha^{\left(\begin{array}{c}
k \\
3
\end{array}\right)}}{\lambda^{\left(\begin{array}{c}
k \\
2
\end{array}\right)}} m^{k}\left(1-\beta^{\prime}\right)|I| .
$$


Since $|I|=\Pi_{B \in[k]^{3}}\left\lfloor\frac{\alpha_{B}}{\alpha}\right\rfloor$, we have from (18) that

$$
\begin{aligned}
& \left|\mathcal{K}_{k}^{(3)}(\mathcal{H})\right| \geq \frac{\alpha^{\left(\begin{array}{c}
k \\
3
\end{array}\right)}}{\lambda^{\left(\begin{array}{c}
k \\
2
\end{array}\right)}} m^{k}\left(1-\beta^{\prime}\right) \Pi_{B \in[k]^{3}}\left\lfloor\frac{\alpha_{B}}{\alpha}\right\rfloor,
\end{aligned}
$$

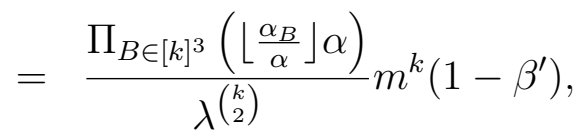

$$
\begin{aligned}
& \geq \frac{\prod_{B \in[k]^{3}}\left(\alpha_{B}-\alpha\right)}{\lambda^{\left(\begin{array}{l}
k \\
2
\end{array}\right)}} m^{k}\left(1-\beta^{\prime}\right), \\
& =\frac{\Pi_{B \in[k]^{3} \alpha_{B}}}{\lambda^{\left(\begin{array}{c}
k \\
2
\end{array}\right)}} m^{k}\left(1-\beta^{\prime}\right) \Pi_{B \in[k]^{3}}\left(1-\frac{\alpha}{\alpha_{B}}\right) .
\end{aligned}
$$

By our choice of the auxiliary constants $\alpha, \beta^{\prime}$ in (11), we infer from (19) that

$$
\left|\mathcal{K}_{k}^{(3)}(\mathcal{H})\right| \geq \frac{\Pi_{B \in[k]^{3} \alpha_{B}}}{\left.\lambda^{(} \begin{array}{c}
k \\
2
\end{array}\right)} m^{k}(1-\beta) .
$$

Hence, (15) is proved, thus proving Claim 3.2.3.

\section{Background Material for the Proof of $R(k-1) \Rightarrow D(k)$}

In this section, we present definitions and technical facts, without proof, needed in Section 5.3 for the proof of Lemma 5.1.1. The proofs of these facts are along technical albeit standard lines. The reader interested in the proofs of these facts is encouraged to see $[6]$.

\subsection{Basic Facts.}

We begin with the following fact from [2].

Fact 4.1.1. Let $M, k, \lambda$ be given integers, and let $\epsilon>0, \sigma \in(0,1]$ be given real numbers. Suppose $G=\bigcup_{0 \leq i<j<k} G^{i j}$ is a $(k+1)$-partite cylinder with $(k+1)$-partition $\left(W_{0}, W_{1}, \ldots, W_{k}\right),\left|W_{1}\right|=\ldots=\left|W_{k}\right|=M$, and $\left|W_{0}\right|=\sigma M$. Suppose that for all $j \in[k]$, $G$ satisfies these conditions:

(i) $G^{0 j}$ induced on $W_{0} \cup W_{j}$ satisfies that whenever $W_{0}^{\prime} \subseteq W_{0}$ and $W_{j}^{\prime} \subseteq W_{j},\left|W_{0}^{\prime}\right| \geq$ $\epsilon M$ and $\left|W_{j}^{\prime}\right| \geq \epsilon M$, then

$$
d_{G^{0 j}}\left(W_{0}^{\prime}, W_{j}^{\prime}\right) \in\left(\frac{1}{\lambda}(1-\epsilon), \frac{1}{\lambda}(1+\epsilon)\right) .
$$

(ii) For all $x \in W_{0},\left|N_{j}(x)\right| \leq \frac{M}{\lambda}(1+\epsilon)$.

Then the following property holds: there exists an integer $M_{0}=M_{0}(\sigma, k, \epsilon)$ such that for all $M \geq M_{0}$, there exist $b \geq \sigma / k \epsilon$ vertices $\left\{x_{1}, \ldots, x_{b}\right\} \subseteq W_{0}$ satisfying $\mid N_{j}\left(x_{u}\right) \cap$ $N_{j}\left(x_{v}\right) \mid \leq \frac{M}{\lambda^{2}}(1+\epsilon)^{2}$, for all $\left(\begin{array}{l}b \\ 2\end{array}\right)$ pairs $\{u, v\} \in[b]^{2}$ and all $j \in[k]$.

We make the following remark about Fact 4.1.1. Suppose that $G$ is a $(\lambda, \epsilon, k+1)$ cylinder with $(k+1)$-partition $\left(V_{0}, V_{1}, \ldots, V_{k}\right),\left|V_{0}\right|=\left|V_{1}\right|=\ldots=\left|V_{k}\right|=M$. Let

$$
V_{0}^{\prime}=\left\{v \in V_{0}:\left|N_{i}(v)\right|>\frac{M}{\lambda}(1+\epsilon) \text {, for some } i \in[k]\right\} \text {. }
$$


By Fact 2.1.5, $\left|V_{0}^{\prime}\right|<k \epsilon M$. Putting $W_{0}=V_{0} \backslash V_{0}^{\prime}, W_{i}=V_{i}$ for all $i \in[k]$, and $\sigma=1-k \epsilon$, we have that the $(k+1)$-partite cylinder $G_{1}$, induced from $G$ on the $(k+1)$-partition $\left(W_{0}, W_{1}, \ldots, W_{k}\right)$, satisfies the hypothesis of Fact 4.1 .1

A consequence of Fact 4.1.1 which is used frequently in Section 5 is stated in the following fact.

Fact 4.1.2. Let $k, r, \lambda$ be given integers, and let $\epsilon>0$, and $\sigma \in(0,1]$ be given real numbers. Suppose $G=\bigcup_{0 \leq i<j \leq k} G^{i j}$ is a $(k+1)$-partite cylinder with $(k+1)$-partition $\left(W_{0}, W_{1}, \ldots, W_{k}\right),\left|W_{1}\right|=\ldots=\left|W_{k}\right|=M$, and $\left|W_{0}\right|=\sigma M$, where $M$ is assumed to be sufficiently large. Suppose that for all $j \in[k], G$ satisfies these conditions:

(i) $G^{0 j}$ induced on $W_{0} \cup W_{j}$ satisfies that whenever $W_{0}^{\prime} \subseteq W_{0}$ and $W_{j}^{\prime} \subseteq W_{j},\left|W_{0}^{\prime}\right| \geq$ $\epsilon M$ and $\left|W_{j}^{\prime}\right| \geq \epsilon M$, then

$$
d_{G^{0 j}}\left(W_{0}^{\prime}, W_{j}^{\prime}\right) \in\left(\frac{1}{\lambda}(1-\epsilon), \frac{1}{\lambda}(1+\epsilon)\right) .
$$

(ii) For all $x \in W_{0},\left|N_{j}(x)\right| \leq \frac{M}{\lambda}(1+\epsilon)$.

Then there exist pairwise disjoint $r$-element subsets $S_{1}, \ldots, S_{q}, q=\left\lceil\frac{\sigma M}{r}(1-r k \epsilon / \sigma)\right\rceil$, satisfying:

(a) for each $i \in[q], S_{i}=\left\{x_{1}^{(i)}, \ldots, x_{r}^{(i)}\right\} \subseteq W_{0}$,

(b) for each $i \in[q]$, for all $\{u, v\} \in[r]^{2}$, and for all $j \in[k]$,

$$
\left|N_{j}\left(x_{u}^{(i)}\right) \cap N_{j}\left(x_{v}^{(i)}\right)\right| \leq \frac{M}{\lambda^{2}}(1+\epsilon)^{2} .
$$

\subsection{Regular Couples.}

We now define a notion of $(\delta, r)$-regularity for cylinders.

Definition 4.2.1. Let $\gamma, \delta$ be positive reals, let $r$ be a positive integer, and let $F$ be a bipartite graph with bipartition $(U, V)$. We say that $F$ is $(\gamma, \delta, r)$-regular if the following property holds: For any $r$-tuple of pairs of subsets $\left(\left\{U_{j}, V_{j}\right\}\right)_{j=1}^{r}, U_{j} \subseteq U, V_{j} \subseteq V$, $1 \leq j \leq r$, satisfying

$$
\left|\bigcup_{j=1}^{r}\left(U_{j} \times V_{j}\right)\right|>\delta|U||V|
$$

then

$$
\frac{\left|F \cap \bigcup_{j=1}^{r}\left(U_{j} \times V_{j}\right)\right|}{\left|\bigcup_{j=1}^{r}\left(U_{j} \times V_{j}\right)\right|}>\gamma
$$

Note that it follows directly from the definition that if $F$ is $(\gamma, \delta, r)$-regular, then $F$ is also $\left(\gamma, \delta^{\prime}, r^{\prime}\right)$-regular for any $\delta^{\prime} \geq \delta$ and positive integer $r^{\prime} \leq r$. We use this fact repeatedly in later sections.

We proceed with the following easy fact.

Fact 4.2.2. Let $\gamma, \delta$, and $r$ be given, and suppose $F$ is a bipartite graph with bipartition $(U, V)$. If $F$ is $(\gamma, \delta, r)$-regular, then all but less than $\delta|U|$ vertices $u \in U$ satisfy

$$
|N(u)|>\gamma|V| \text {. }
$$


When we encounter $(\gamma, \delta, r)$-regular bipartite graphs, they actually are subgraphs of other highly regular bipartite graphs. This situation prompts the following definition.

Definition 4.2.3. Let $\gamma, \delta, \epsilon$ be given positive reals, let $r, \lambda$ be given positive integers, and let $F, G$ be two given bipartite graphs, each with bipartition $(U, V)$. We call the ordered pair of graphs $(F, G)$ a $(\gamma, \delta, r, \lambda, \epsilon)$-regular couple provided

(i) $F \subseteq G$,

(ii) $F$ is $(\gamma, \delta, r)$-regular,

(iii) $G$ is a $(\lambda, \epsilon, 2)$-cylinder.

Note that in the definition above, $F$ being $(\gamma, \delta, r)$-regular only ensures a lower bound of $\gamma$ on the density described in (20). With $G$ being a $(\lambda, \epsilon, k)$-cylinder, Definition 2.1.4 ensures that the density of bipartite subgraphs of $G$ on subsets $U^{\prime} \subseteq U,\left|U^{\prime}\right|>\epsilon|U|$, $V^{\prime} \subseteq V,\left|V^{\prime}\right|>\epsilon|V|$, is roughly $\frac{1}{\lambda}$.

The following important fact relates to Fact 2.1.7.

Fact 4.2.4. For all integers $k \geq 3$ and all positive $\alpha, \beta$, there exists $\delta>0$ so that for all integers $\lambda \geq \frac{1}{\delta}$, there exist $r, \epsilon$ so that the following property holds: Suppose

(i) $G=\bigcup_{1 \leq i<j \leq k} G^{i j}$ is a $(\lambda, \epsilon, k)$-cylinder with $k$-partition $\left(V_{1}, \ldots, V_{k}\right),\left|V_{1}\right|=\ldots=$ $\left|V_{k}\right|=m$.

(ii) $F=\bigcup_{1 \leq i<j \leq k} F^{i j}$ is a $k$-partite cylinder defined on the same $k$-partition $\left(V_{1}, \ldots, V_{k}\right)$.

(iii) For all $i, j, 1 \leq i<j \leq k,\left(F^{i j}, G^{i j}\right)$ is a $\left(\frac{\alpha-2 \delta}{\lambda}, \delta, r, \lambda, \epsilon\right)$-regular couple.

Then $F$ satisfies

$$
\left|\mathcal{K}_{k}^{(2)}(F)\right| \geq\left(\frac{\alpha}{\lambda}\right)^{\left(\begin{array}{l}
k \\
2
\end{array}\right)} m^{k}(1-\beta)
$$

\subsection{Link Graphs.}

For our final fact, we need the following definition.

Definition 4.3.1. Let $\mathcal{H}$ be a $k$-partite 3 -cylinder with $k$-partition $\left(V_{1}, \ldots, V_{k}\right)$, and let $G=\bigcup_{1 \leq i<j \leq k} G^{i j}$ be an underlying $k$-partite cylinder. Let $i, j, 1<i<j \leq k$, be integers and let $x \in V_{1}$. We define the $\{i, j\}$-link graph of $x, L_{x}^{i j}$, as

$$
L_{x}^{i j}=\left\{\{y, z\} \in G^{i j}: y \in N_{G, i}(x), z \in N_{G, j}(x),\{x, y, z\} \in \mathcal{H}\right\} .
$$

We further define the link graph of $x, L_{x}$, as

$$
L_{x}=\bigcup_{1<i<j \leq k} L_{x}^{i j}
$$

Before continuing with our next fact, we describe some notation we need. Suppose $G=\bigcup_{1<i<j \leq k} G^{i j}$ is a $k$-partite cylinder with $k$-partition $\left(V_{1}, \ldots, V_{k}\right)$, and let $x \in V_{1}$. For fixed $i, j, 1<i<j \leq k$, define

$$
G^{i j}(x, G)=\left\{\{x, y\} \in G^{i j}: x \in N_{G, i}(x), y \in N_{G, j}(x)\right\}=G^{i j}\left[N_{G, i}(v), N_{G, j}(v)\right] .
$$

We conclude this section by stating that any $(\alpha, \delta, r)$-regular hypergraph $\mathcal{H}$ with underlying $(\lambda, \epsilon, k)$-cylinder $G=\bigcup_{1 \leq i<j \leq k} G^{i j}$ (on partite sets $\left(V_{1}, \ldots, V_{k}\right)$ ) satisfies that for all $i, j, 1<i<j \leq k$, and most vertices $x \in V_{1}$, the $\{i, j\}$-link graph $L_{x}^{i j}$ is $\left(\frac{\alpha-2 \sqrt{\delta}}{\lambda}, \sqrt{\delta}, r\right)$-regular. We now present this crucial fact. 
Fact 4.3.2. For all positive reals $\alpha, \delta, \alpha>2 \delta$, and for all positive integers $k, \lambda$, and $r$, there exists $\epsilon>0$ so that the following property holds: suppose

(i) $\mathcal{H}$ is a k-partite 3-cylinder with k-partition $\left(V_{1}, \ldots, V_{k}\right),\left|V_{1}\right|=\ldots=\left|V_{k}\right|=m$.

(ii) $G=\bigcup_{1 \leq i<j \leq k} G^{i j}$ is an underlying $(\lambda, \epsilon, k)$-cylinder of $\mathcal{H}$.

(iii) For all $i, j, 1<i<j \leq k, \mathcal{H}(\{1, i, j\})$ is $(\alpha, \delta, r)$-regular with respect to $G(\{1, i, j\})$.

Then

(a) all but $4 \epsilon(k-1) m$ vertices

$v \in V_{1}$ satisfy that for each $i \in\{2, \ldots, k\}$,

$$
\frac{m}{\lambda}(1-\epsilon) \leq\left|N_{G, i}(v)\right| \leq \frac{m}{\lambda}(1+\epsilon),
$$

(b) all but $2\left(\begin{array}{c}k-1 \\ 2\end{array}\right) \sqrt{\delta} m$ vertices

$v \in V_{1}$ satisfy that for each $i, j, 1<i<j \leq k,\left(L_{x}^{i j}, G^{i j}(x, G)\right)$ is an $\left(\frac{\alpha-2 \sqrt{\delta}}{\lambda}, \sqrt{\delta}, r, \lambda, 2 \lambda \epsilon\right)$-regular couple.

$$
\text { 5. } R(k-1) \Rightarrow D(k)
$$

The objective of this section is to prove that $R(k-1) \Rightarrow D(k)$. To that end, we give an auxiliary statement in Lemma 5.1.1 that implies $R(k-1) \Rightarrow D(k)$. In Claim 5.1.2, we prove the sufficiency of Lemma 5.1.1 for the implication $R(k-1) \Rightarrow D(k)$. Afterwards, we verify Lemma 5.1.1. Note that our proof of Lemma 5.1.1 largely follows from principles given in the previous section.

5.1. Lemma 5.1.1 and $R(k-1) \Rightarrow D(k)$.

We now state Lemma 5.1.1.

Lemma 5.1.1. For all integers $k \geq 4$, for all $\alpha>0$ and $\beta>0$, there exists a positive constant $\delta$ so that for all integers $\lambda \geq \frac{1}{\delta}$, there exist $r, \epsilon$ such that the following holds: suppose

(i) $\mathcal{H}$ is a k-partite 3-cylinder with k-partition $\left(V_{1}, \ldots, V_{k}\right),\left|V_{1}\right|=\ldots=\left|V_{k}\right|=m$.

(ii) $G$ is an underlying $(\lambda, \epsilon, k)$-cylinder of $\mathcal{H}$.

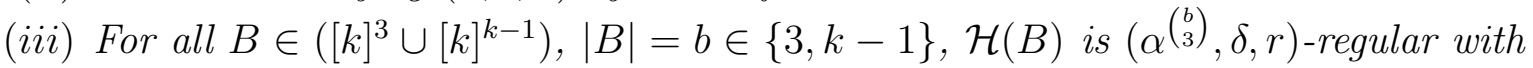
respect to $G(B)$.

Then

$$
\left|\mathcal{K}_{k}^{(3)}(\mathcal{H})\right| \geq \frac{\alpha^{\left(\begin{array}{c}
k \\
3
\end{array}\right)}}{\lambda^{\left(\begin{array}{c}
k \\
2
\end{array}\right)}} m^{k}(1-\beta) .
$$

We now prove in Claim 5.1.2 that in order to show the implication $R(k-1) \Rightarrow D(k)$, it is enough to show Lemma 5.1.1.

Claim 5.1.2. For $k \geq 4$, Lemma 5.1 .1 implies the implication $R(k-1) \Rightarrow D(k)$.

Before proceeding to the proof of Claim 5.1.2, we first discuss the main ideas behind the proof of Claim 5.1.2. Fix $k \geq 4$, and assume both Lemma 5.1.1 and the statement $R(k-1)$ are true. Our goal is to show that the statement $D(k)$ is true for the fixed 
integer $k \geq 4$. Before formally disclosing the constants $\delta, r$, and $\epsilon$ guaranteed to exist by statement $D(k)$, we reveal our strategy for proving $D(k)$.

Note that in the hypothesis of $D(k)$, we assume that all $\mathcal{H}(B), B \in[k]^{3}$, are sufficiently $(\alpha, \delta, r)$-regular. The statement $R(k-1)$ being true ensures us that having all $\mathcal{H}(B)$ sufficiently regular guarantees that all $(k-1)$-partite 3 -cylinders $\mathcal{H}(C), C \in[k]^{k-1}$, are arbitrarily regular. Lemma 5.1 .1 being true ensures us that with both the triads $\mathcal{H}(B)$ and the $(k-1)$-partite 3 -cylinders $\mathcal{H}(C)$ sufficiently regular, $\mathcal{H}$ must contain at least

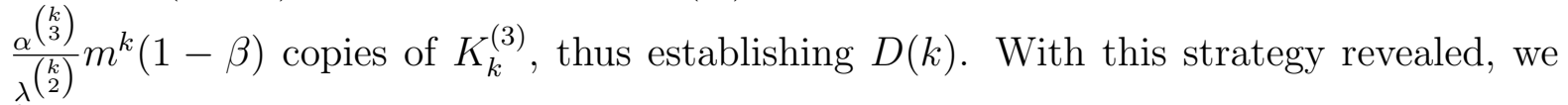
formally disclose the constants.

\subsection{Proof of Claim 5.1.2.}

With $k \geq 4$ already given, let $\alpha, \beta>0$ be given. Our first goal is to define the constants guaranteed by the statement $D(k)$, starting with the promised constant $\delta$.

\section{Definitions of the Constants:}

We first define the constant $\delta$ promised by the statement $D(k)$. Recall that we assume that Lemma 5.1.1 is true for the fixed integer $k \geq 4$. Choose the constants $\alpha$ and $\beta$ as they are given above. Lemma 5.1.1 guarantees a constant $\delta_{L}=\delta_{L}(\alpha, \beta)$. Recall we also assume that the statement $R(k-1)$ is true for the fixed integer $k \geq 4$. Choose $\alpha$ as it was given above, and let $\delta_{k-1}=\delta_{L}$. The statement $R(k-1)$ guarantees a constant $\delta_{R}=\delta_{R}\left(\alpha, \delta_{L}\right)$. Note that $\delta_{R} \leq \delta_{L}$. Set $\delta=\delta_{R}$. Thus, we have produced the constant $\delta$ promised by the statement $D(k)$.

Let $\lambda \geq \frac{1}{\delta}$ be given. Our next goal is to produce the constants $r$ and $\epsilon$ promised by the statement $D(k)$. Again, recall that we assume Lemma 5.1.1 and the statement $R(k-1)$ are true for the fixed integer $k \geq 4$.

For the constants $\alpha$ and $\beta$ given above, $\delta=\delta_{R}$, and $\lambda \geq \frac{1}{\delta}$ chosen above, Lemma 5.1.1 guarantees constants $r_{L}=r_{L}(\alpha, \beta, \delta, \lambda)$ and $\epsilon_{L}=\epsilon_{L}(\alpha, \beta, \delta, \lambda)$.

Set $r_{k-1}=r_{L}$. With constants $\alpha, \delta_{k-1}=\delta_{L}, \delta, \lambda, r_{k-1}=r_{L}$ determined above, the statement $R(k-1)$ guarantees constants $r_{R}=r_{R}\left(\alpha, \delta_{L}, \delta, \lambda, r_{L}\right)$ and $\epsilon_{R}=\epsilon_{R}\left(\alpha, \delta_{L}, \delta, \lambda, r_{L}\right)$. Set

$$
r=\max \left\{r_{L}, r_{R}\right\}
$$

and

$$
\epsilon=\min \left\{\epsilon_{L}, \epsilon_{R}\right\}
$$

Thus, we have determined the constants guaranteed by the statement $D(k)$. We now prove that $D(k)$ is true with the constants $\delta, r$ and $\epsilon$ defined above.

\section{Proof of Claim 5.1.2.}

As in the hypothesis of statement $D(k)$, suppose that $k \geq 4, \alpha$ and $\beta>0$ are given constants. Let the constant $\delta$ be given in the Definitions of the Constants. Let $\lambda>\frac{1}{\delta}$ be a given integer, and suppose constants $r$ and $\epsilon$ are given in the Definitions of the Constants. Suppose triple system $\mathcal{H}$ and underlying graph $G$ satisfy the following setup with constants $k, \alpha, \delta, \lambda, r$ and $\epsilon$ :

(i) $\mathcal{H}$ is a $k$-partite 3 -cylinder with $k$-partition $\left(V_{1}, \ldots, V_{k}\right),\left|V_{1}\right|=\ldots=\left|V_{k}\right|=m$.

(ii) Let $G$ be an underlying $(\lambda, \epsilon, k)$-cylinder of $\mathcal{H}$. 
(iii) For all $B \in[k]^{3}, \mathcal{H}(B)$ is $(\alpha, \delta, r)$-regular with respect to the triad $G(B)$.

In order to prove the statement $D(k)$ is true, we must show

$$
\left|\mathcal{K}_{k}^{(3)}(\mathcal{H})\right| \geq \frac{\alpha^{\left(\begin{array}{c}
k \\
3
\end{array}\right)}}{\lambda^{\left(\begin{array}{c}
k \\
2
\end{array}\right)}} m^{k}(1-\beta) .
$$

Let $C \in[k]^{k-1}$, and consider the restricted $(k-1)$-partite 3 -cylinder $\mathcal{H}(C)$ with underlying restricted $(\lambda, \epsilon, k-1)$-cylinder $G(C)$. We first prepare the $(k-1)$-partite 3-cylinder $\mathcal{H}(C)$ with underlying $(\lambda, \epsilon, k-1)$-cylinder $G(C)$ for the statement $R(k-1)$. Specifically, note that all triads $\mathcal{H}(B)$ of $\mathcal{H}(C), B \in[C]^{3}$, are $(\alpha, \delta, r)$-regular, where $\delta, r$, and $\epsilon$ satisfy the following: with $\delta_{k-1}=\delta_{L}$, we have $\delta=\delta_{R}\left(\alpha, \delta_{L}\right)$. With $\lambda \geq \frac{1}{\delta}$, we have $r \geq r_{R}$ and $\epsilon \leq \epsilon_{R}$. Thus, all triads $\mathcal{H}(B)$ of $\mathcal{H}(C)$ are $\left(\alpha, \delta_{R}, r_{R}\right)$-regular with respect to the $\left(\lambda, \epsilon_{R}, 3\right)$-cylinder $G(B)$, where $\delta_{R}, r_{R}$, and $\epsilon_{R}$, are parameters which verify the applicability of statement $R(k-1)$ for the choices $\alpha, \delta_{k-1}=\delta_{L}, \lambda \geq \frac{1}{\delta}$, and $r_{k-1}=r_{L}$. The statement $R(k-1)$ guarantees that the $(k-1)$-partite 3 -cylinder $\mathcal{H}(C)$ is $\left(\alpha^{\left(\begin{array}{c}k-1 \\ 3\end{array}\right)}, \delta_{L}, r_{L}\right)$-regular with respect to the $(\lambda, \epsilon, k-1)$-cylinder $G(C)$. This property holds for all $C \in[k]^{k-1}$.

Now we prepare the $k$-partite 3 -cylinder $\mathcal{H}$ with underlying $(\lambda, \epsilon, k)$-cylinder $G$ for Lemma 5.1.1. By assumption, all triads $\mathcal{H}(B), B \in[k]^{3}$, are $(\alpha, \delta, r)$-regular with respect to $G(B)$, so in particular, with $\delta=\delta_{R} \leq \delta_{L}$ and $r \geq r_{L}, r_{R}$, all triads $\mathcal{H}(B)$ are both $\left(\alpha, \delta_{R}, r_{R}\right)$-regular and $\left(\alpha, \delta_{L}, r_{L}\right)$-regular with respect to $G(B)$. By the application of $R(k-1)$ above, since all triads $\mathcal{H}(B)$ are $\left(\alpha, \delta_{R}, r_{R}\right)$-regular, we also conclude that for all $C \in[k]^{k-1}$, the $(k-1)$-partite 3 -cylinder $\mathcal{H}(C)$ is $\left(\alpha^{\left(\begin{array}{c}k-1 \\ 3\end{array}\right)}, \delta_{L}, r_{L}\right)$-regular with respect to the $\left(\lambda, \epsilon_{L}, k-1\right)$-cylinder $G(C)$. Since all triads $\mathcal{H}(B)$ are $\left(\alpha, \delta_{L}, r_{L}\right)$-regular, as well as all $(k-1)$-partite 3 -cylinders $\mathcal{H}(C)$ are $\left(\alpha^{\left(\begin{array}{c}k-1 \\ 3\end{array}\right)}, \delta_{L}, r_{L}\right)$-regular, where $\delta_{L}, r_{L}$, and $\epsilon_{L}$ verify the applicability of Lemma 5.1.1 for the choices $\alpha, \beta$, and $\lambda$, we have the hypothesis of Lemma 5.1.1 satisfied. By that lemma,

$$
\left|\mathcal{K}_{k}^{(3)}(\mathcal{H})\right| \geq \frac{\alpha^{\left(\begin{array}{c}
k \\
3
\end{array}\right)}}{\lambda^{\left(\begin{array}{c}
k \\
2
\end{array}\right)}} m^{k}(1-\beta) .
$$

Thus the statement $D(k)$ is true for the fixed integer $k \geq 4$.

\subsection{Proof of Lemma 5.1.1.}

We conclude this section by proving Lemma 5.1.1. We begin by defining the constants involved.

\section{Definitions of the Constants:}

Let $k \geq 3$ be an arbitrary integer, and $\alpha, \beta$ be arbitrary positive constants. Before precisely defining the constants $\delta, r$ and $\epsilon$ guaranteed to exist by Lemma 5.1.1, we first informally introduce these and other constants, and emphasize the hierarchy which governs their relative sizes. With $\alpha, \beta$ given, we first define constants $\gamma, \delta_{1}, \delta_{2}$, and $\delta$ so that

$$
\alpha \gg \gamma \gg \min \left\{\delta_{1}, \delta_{2}^{2}\right\}=\delta
$$


For $\lambda \geq \frac{1}{\delta}$, we next define constants $r_{1}, r_{2}, r, \epsilon_{1}, \epsilon_{2}, \epsilon_{3}$, and $\epsilon$ so that

$$
\frac{1}{\lambda} \gg \min \left\{\frac{1}{r_{1}}, \frac{1}{r_{2}}\right\}=\frac{1}{r} \gg \min \left\{\frac{\epsilon_{1}}{2 \lambda}, \epsilon_{2}, \epsilon_{3}\right\}=\epsilon .
$$

We now define these constants formally, however, for quick reference, one may think of the following hierarchy,

$$
\alpha \gg \gamma \gg \sqrt{\delta} \gg \delta>\frac{1}{\lambda} \gg \frac{1}{r} \gg \epsilon,
$$

as it is this hierarchy which justifies all of our calculations in the proof of Lemma 5.1.1.

With $\alpha, \beta$ given positive constants, we first define an auxiliary positive constant $\bar{\beta}$ to satisfy

$$
1-\bar{\beta}>(1-\beta)^{1 / 6} \text {. }
$$

Further define auxiliary positive constant $\gamma$ to satisfy both

$$
\begin{aligned}
\sqrt{\gamma} & \leq \frac{\alpha^{\left(\begin{array}{c}
k-1 \\
2
\end{array}\right)(1-\bar{\beta})}}{4 \cdot 8^{k-1}}, \\
1-\sqrt{\gamma} & >(1-\beta)^{1 / 6} .
\end{aligned}
$$

We now produce the constant $\delta$ promised in Lemma 5.1.1. Let $\delta_{1}>0$ satisfy

$$
\begin{aligned}
1-\frac{2 \delta_{1}}{\alpha^{\left(\begin{array}{c}
k-1 \\
3
\end{array}\right)}} & >(1-\beta)^{1 / 6}, \\
1-5 \sqrt{\delta_{1}\left(\begin{array}{c}
k-1 \\
2
\end{array}\right)} & >(1-\beta)^{1 / 6}, \\
2 \delta_{1} & <\frac{\gamma(1-\sqrt{\gamma})(1-\bar{\beta}) \alpha^{\left(\begin{array}{c}
k-1 \\
2
\end{array}\right)}}{2^{k}} .
\end{aligned}
$$

We want to ensure that our choice of $\delta$ is sufficient for an application of Fact 4.2.4. For the constants $\alpha, \bar{\beta}$, and $k-1$ from above, let

$$
\delta_{2}=\delta_{2}(\alpha, \bar{\beta}, k-1)
$$

be the constant guaranteed by Fact 4.2.4. Set

$$
\delta=\min \left\{\delta_{1}, \delta_{2}^{2}\right\} .
$$

Let $\lambda \geq \frac{1}{\delta}=\max \left\{\frac{1}{\delta_{1}}, \frac{1}{\delta_{2}^{2}}\right\}$ be a given integer. Fact 4.2.4 guarantees the existence of constants

$$
\begin{aligned}
& \epsilon_{1}=\epsilon_{1}(\alpha, \bar{\beta}, k-1, \delta, \lambda) \\
& r_{1}=r_{1}(\alpha, \bar{\beta}, k-1, \delta, \lambda) .
\end{aligned}
$$

Let

$$
r_{2}=\left\lceil\gamma \lambda^{k-1}\right\rceil
$$

and

$$
r=\max \left\{r_{1}, r_{2}\right\}
$$


Finally, we define the constant $\epsilon$ guaranteed by Lemma 5.1.1. In order to define $\epsilon$, we need to first define additional constants $\epsilon_{2}$ and $\epsilon_{3}$. We want to ensure that our choice of $\epsilon$ will be sufficient for an application of Fact 4.3.2. With constants $k, \alpha, \delta, \lambda$, and $r$ set above, let

$$
\epsilon_{2}=\epsilon_{2}(\alpha, \delta, k, \lambda, r)
$$

be that constant guaranteed by Fact 4.3.2.

Recall the function $\theta_{k, \lambda}(\epsilon)$ defined in Fact 2.1.7. This function has the property that for $\lambda$ fixed, $\theta_{k, \lambda}(\epsilon) \rightarrow 0$ as $\epsilon \rightarrow 0$. Let $\epsilon_{3}$ be a positive constant satisfying each of the inequalities below

$$
\begin{aligned}
\epsilon_{3} & <\frac{\sqrt{\delta}}{2} \\
\theta_{k-1, \lambda}\left(2 \lambda^{2} \epsilon_{3}\right) & <1 \\
\left(1-\epsilon_{3}\right)^{k-1} & >(1-\beta)^{1 / 6}, \\
1-\frac{(k-1) r \epsilon_{3}}{1-4 \sqrt{\delta}\left(\begin{array}{c}
k-1 \\
2
\end{array}\right)} & >(1-\beta)^{1 / 6} .
\end{aligned}
$$

Having defined the constants $\epsilon_{1}, \epsilon_{2}$, and $\epsilon_{3}$, set

$$
\epsilon=\min \left\{\frac{\epsilon_{1}}{2 \lambda}, \epsilon_{2}, \epsilon_{3}\right\} .
$$

Note that the constants $\alpha, \gamma, \lambda, r$, and $\epsilon$ defined above do indeed satisfy the following hierarchy

$$
\alpha \gg \gamma \gg \sqrt{\delta} \gg \delta>\frac{1}{\lambda} \gg \frac{1}{r} \gg \epsilon .
$$

We see that the first 2 inequalities follow from (25) and (29), (31). We see in (35) that $r \gg \lambda$, and the last inequality follows from (40) and (41).

Having defined all the promised constants, we proceed with the proof of Lemma 5.1.1. Proof of Lemma 5.1.1.

Let $\alpha, \beta>0$ and integer $k \geq 4$ be given. Let $\delta$ be given in (31). Let integer $\lambda>\frac{1}{\delta}$ be given. Let $r, \epsilon$ be given in (35) and (41) respectively. Let $\mathcal{H}$ be a $k$-partite 3 -cylinder with $k$-partition $\left(V_{1}, \ldots, V_{k}\right),\left|V_{1}\right|=\ldots=\left|V_{k}\right|=m$, and let $G=\cup_{1 \leq i<j \leq k} G^{i j}$ be an underlying $(\lambda, \epsilon, k)$-cylinder of $\mathcal{H}$. Suppose further that for all $B \in\left([k]^{3} \cup[k]^{k-1}\right)$, $|B|=b \in\{3, k-1\}, \mathcal{H}(B)$ is $\left(\alpha^{\left(\begin{array}{c}b \\ 3\end{array}\right)}, \delta, r\right)$-regular with respect to $G(B)$. Our goal is to show that $\mathcal{H}$ satisfies

We first state a definition.

$$
\left|\mathcal{K}_{k}^{(3)}(\mathcal{H})\right| \geq \frac{\alpha^{\left(\begin{array}{c}
k \\
3
\end{array}\right)}}{\lambda^{\left(\begin{array}{c}
k \\
2
\end{array}\right)}} m^{k}(1-\beta) .
$$

Definition 5.3.1. Let $v \in V_{1}$ be a vertex from the vertex set $V_{1}$. We say that $v$ is a good vertex provided that $v$ satisfies the following conditions:

(i) For all $i, 1<i \leq k$,

$$
\frac{m}{\lambda}(1-\epsilon)<\left|N_{G, i}(v)\right|<\frac{m}{\lambda}(1+\epsilon) .
$$


(ii) For all $i, j, 1<i<j \leq k$, the pair of graphs $\left(L_{v}^{i j}, G^{i j}(v, G)\right)$, the $\{i, j\}$-link graph of $v$ and the subgraph of $G^{i j}$ induced on the sets $N_{G, i}(v)$ and $N_{G, j}(v)$, form an $\left(\frac{\alpha-2 \sqrt{\delta}}{\lambda}, \sqrt{\delta}, r, \lambda, 2 \lambda \epsilon\right)$-regular couple.

Set

$$
V_{1}^{\prime}=\left\{v \in V_{1}: v \text { is a good vertex }\right\} .
$$

We prove the following claim.

Claim 5.3.2.

$$
\left|V_{1}^{\prime}\right| \geq\left(1-4 \sqrt{\delta}\left(\begin{array}{c}
k-1 \\
2
\end{array}\right)\right) m
$$

\section{Proof of Claim 5.3.2.}

We would like to apply Fact 4.3.2 to $G$ to infer that the size of $V_{1}^{\prime}$ is approximately the size of $V_{1}$. First, we note that the hypothesis of Fact 4.3.2 is satisfied by $\mathcal{H}$ and $G$. Indeed, by assumption, $\mathcal{H}$ and $G$ satisfy that all $\left(\begin{array}{l}k \\ 3\end{array}\right)$ triads are $(\alpha, \delta, r)$-regular, and that $G$ is a $(\lambda, \epsilon, k)$-cylinder with $\epsilon \leq \epsilon_{2}$. Since $\epsilon_{2}$ is set in (36) to verify the applicability of Fact 4.3.2 for the parameters $k, \alpha, \delta, \lambda$, and $r$, and since $\epsilon \leq \epsilon_{2}$, then $\epsilon$ also verifies the applicability of Fact 4.3.2 for the same parameters. Statement $(a)$ of Fact 4.3.2 tells us that all but $4(k-1) \epsilon m$ vertices satisfy (42) for all $i, 1 \leq i \leq k$. Statement $(b)$ of Fact 4.3.2 tells us that for fixed $i, j, 1<i<j \leq k$, all but $2\left(\begin{array}{c}k-1 \\ 2\end{array}\right) \sqrt{\delta} m$ vertices $x \in V_{1}$ satisfy that $\left(L_{x}^{i j}, G^{i j}(x, G)\right)$ is an $\left(\frac{\alpha-2 \sqrt{\delta}}{\lambda}, \sqrt{\delta}, r, \lambda, 2 \lambda \epsilon\right)$-regular couple. Thus, we have

$$
\begin{aligned}
\left|V_{1}^{\prime}\right| & >\left(1-\left(\begin{array}{c}
k-1 \\
2
\end{array}\right) 2 \sqrt{\delta}-4(k-1) \epsilon\right) m, \\
& >\left(1-4 \sqrt{\delta}\left(\begin{array}{c}
k-1 \\
2
\end{array}\right)\right) m
\end{aligned}
$$

where the last inequality follows from the fact that we chose $\epsilon$ in (37) and (41) to satisfy $\epsilon<\frac{\sqrt{\delta}}{2}$.

For convenience, set

$$
\sigma=1-4 \sqrt{\delta}\left(\begin{array}{c}
k-1 \\
2
\end{array}\right)
$$

so that we may simply say $\left|V_{1}^{\prime}\right|>\sigma m$.

We now pause to reveal our strategy for the proof of Lemma 5.1.1. Our main technique in this proof is counting copies of $K_{k-1}^{(2)}$ in the link graphs $L_{v}$ of good vertices $v \in V_{1}^{\prime}$. It follows from the definition of the link graph $L_{v}$ that any copy of $K_{k-1}^{(2)} \subset L_{v}$ corresponds to a copy of $K_{k}^{(3)} \subset \mathcal{H}$. Hence, obtaining a lower bound on the total number of copies of $K_{k-1}^{(2)}$ contained within the link graphs $L_{v}, v \in V_{1}^{\prime}$, implies a lower bound on the number of copies of $K_{k}^{(3)}$ contained within $\mathcal{H}$. This lower bound will match the one promised by Lemma 5.1.1.

We consider good vertices in our argument outlined above for the following reason. A good vertex $v$ satisfies that for all $i, j, 1<i<j \leq k,\left(L_{v}^{i j}, G^{i j}(v, G)\right)$ is a 
$\left(\frac{\alpha-2 \sqrt{\delta}}{\lambda}, \sqrt{\delta}, r, \lambda, 2 \lambda \epsilon\right)$-regular couple, and therefore (as we prove in Claim 5.3.4) satisfies the hypothesis of Fact 4.2.4. Fact 4.2.4 then provides a lower bound on the number of copies of $K_{k-1}^{(2)}$ contained within $L_{v}$.

We proceed by proving the following claim which will be useful in the strategy above.

Claim 5.3.3. There exists a family $\mathcal{S}=\left\{S_{1}, \ldots, S_{q}\right\}$ of pairwise disjoint, $r_{2}$-element subsets of $V_{1}^{\prime}$ which satisfies:

(i) $q \geq \frac{\sigma m}{r_{2}}\left(1-\frac{(k-1) r_{2} \epsilon}{\sigma}\right)$, where $\sigma$ is given in (43),

(ii) for each $i \in[q], S_{i}=\left\{v_{1}^{(i)}, v_{2}^{(i)}, \ldots, v_{r_{2}}^{(i)}\right\} \subset V_{1}^{\prime}$,

(iii) for each $i \in[q]$, for each $\{x, y\} \in\left[r_{2}\right]^{2}$, for all $j, 1<j \leq k$,

$$
\left|N_{G, j}\left(v_{x}^{(i)}\right) \cap N_{G, j}\left(v_{y}^{(i)}\right)\right| \leq \frac{m}{\lambda^{2}}(1+\epsilon)^{2} .
$$

\section{Proof of Claim 5.3.3.}

We apply Fact 4.1 .2 to the subgraph $\widetilde{G}$ of $G$ induced on the sets $V_{1}^{\prime}, V_{2}, \ldots, V_{k}$. Fact 4.1.2 would guarantee the existence of a family $\mathcal{S}$ described precisely in Claim 5.3.3, so we need only check that the hypothesis of Fact 4.1.2 is met. To that effect, given the notation used in Fact 4.1.2, set $V_{1}^{\prime}=W_{0}, V_{2}=W_{1}, \ldots, V_{k}=W_{k-1}, m=M$, and let $\sigma$ be given as in (43). Under this translation of notation, we write $\widetilde{G}=\bigcup_{0 \leq i<j \leq k-1} \widetilde{G^{i j}}$, where for all $i, j, 0<i<j \leq k-1, \widetilde{G^{i j}}=G\left[W_{i}, W_{j}\right]=G\left[V_{i+1}, V_{j+1}\right]$, and for $0=i<j \leq k-1$, $\widetilde{G^{0 j}}=G\left[W_{0}, W_{j}\right]=G\left[V_{1}^{\prime}, V_{j+1}\right]$. The condition of Fact 4.1 .2 in $(i)$ requires that for every $j \in[k-1]$, the graph $\widetilde{G^{0 j}}$ satisfies that for every $W_{0}^{\prime} \subseteq W_{0},\left|W_{0}^{\prime}\right| \geq \epsilon M$ and for every $W_{j}^{\prime} \subseteq W_{j},\left|W_{j}^{\prime}\right| \geq \epsilon M$, then

$$
d_{\widetilde{G^{0 j}}}\left(W_{0}^{\prime}, W_{j}^{\prime}\right) \in\left(\frac{1}{\lambda}(1-\epsilon), \frac{1}{\lambda}(1+\epsilon)\right) .
$$

However, this condition is easily satisfied by the fact that $G$ is a $(\lambda, \epsilon, k)$ cylinder. The condition of Fact 4.1.2 (ii) requires that all $v \in W_{0}=V_{1}^{\prime}$ satisfy that for all $j \in[k-1]$,

$$
\left|N_{\widetilde{G}, j}(v)\right| \leq \frac{M}{\lambda}(1+\epsilon) .
$$

However, this is satisfied by the fact that all $v \in V_{1}^{\prime}$ satisfy the property in (42) of Definition 5.3.1. Thus, we may apply Fact 4.1.2, and hence obtain a family $\mathcal{S}$ described precisely in Claim 5.3.3 above.

Let $\mathcal{S}=\left\{S_{1}, \ldots, S_{q}\right\}$ be that collection guaranteed by Claim 5.3 .3 above satisfying (i), $($ ii $)$, and $($ iii $)$ in the statement of Claim 5.3.3. From the collection $\mathcal{S}$, consider the set $S_{1}=\left\{v_{1}^{(1)}, v_{2}^{(1)}, \ldots, v_{r_{2}}^{(1)}\right\} \in \mathcal{S}$. For convenience of notation, we drop the superscripts to obtain $S_{1}=\left\{v_{1}, v_{2}, \ldots, v_{r_{2}}\right\}$. For $\mu \in\left[r_{2}\right]$, fix vertex $v=v_{\mu} \in S_{1}$, and consider the link graph $L_{v}=\bigcup_{1<i<j \leq k} L_{v}^{i j}$. We conclude a lower bound on $\left|\mathcal{K}_{k-1}^{(2)}\left(L_{v}\right)\right|$ in the following claim.

Claim 5.3.4. For the fixed vertex $v \in S_{1}$,

$$
\left|\mathcal{K}_{k-1}^{(2)}\left(L_{v}\right)\right| \geq\left(\frac{\alpha}{\lambda}\right)^{\left(\begin{array}{c}
k-1 \\
2
\end{array}\right)}\left(\frac{m}{\lambda}(1-\epsilon)\right)^{k-1}(1-\bar{\beta}) .
$$




\section{Proof of Claim 5.3.4.}

We apply Fact 4.2 .4 to the pair of graphs $L_{v}=\bigcup_{1<i<j \leq k} L_{v}^{i j}$ and $\bigcup_{1<i<j \leq k} G^{i j}(v, G)$. Note that the hypothesis of Fact 4.2.4 is met with this pair of graphs. Indeed, the graph $\bigcup_{1<i<j \leq k} G^{i j}(v, G)$ is a $(\lambda, 2 \lambda \epsilon, k-1)$-cylinder with $(k-1)$-partition $\left(N_{G, 2}(v), \ldots, N_{G, 3}(v)\right)$, where by virtue of $v \in V_{1}^{\prime}$ being a good vertex, the inequality $\left|N_{G, i}(v)\right|>\frac{m}{\lambda}(1-\epsilon)$ from (42) is satisfied for all $i, 1<i \leq k$. The graph $L_{v}=\bigcup_{1<i<j \leq k} L_{v}^{i j}$ is a $(k-1)$-partite cylinder defined on the same $(k-1)$-partition, where for all $i, j, 1<i<j \leq k$, $L_{v}^{i j} \subseteq G^{i j}(v, G)$. Since by virtue of $v \in V_{1}^{\prime}$ being a good vertex, the pair $\left(L_{v}^{i j}, G^{i j}(v, G)\right)$ is a $\left(\frac{\alpha-2 \sqrt{\delta}}{\lambda}, \sqrt{\delta}, r, \lambda, 2 \lambda \epsilon\right)$-regular couple for all $i, j, 1<i<j \leq k$ (cf., Definition 4.2.3). The only condition left to check before applying Fact 4.2 .4 is that the constants $\delta, r$, and $\epsilon$ are appropriate to invoke Fact 4.2.4.

Recall $\delta_{2}=\delta_{2}(\alpha, \bar{\beta}, k-1)$ was that constant set in (30) to verify the applicability of Fact 4.2.4 for the parameters $\alpha, \bar{\beta}$, and $k-1$. Similarly, $r_{1}=r_{1}(\alpha, \bar{\beta}, k-1, \delta, \lambda)$ and $\epsilon_{1}=\epsilon_{1}(\alpha, \bar{\beta}, k-1, \delta, \lambda)$ were those constants set in (33) and (32) respectively to verify the applicability of Fact 4.2.4 for the parameters $\alpha, \bar{\beta}, k-1, \delta$, and $\lambda, \lambda \geq \frac{1}{\delta}$. Since $\sqrt{\delta} \leq \delta_{2}$ in (31), $r \geq r_{1}$ in (35), and $\epsilon \leq \frac{\epsilon_{1}}{2 \lambda}$ in (41), we have that $\sqrt{\delta}, r$, and $2 \lambda \epsilon$ verifies the applicability of Fact 4.2.4 for the same parameters.

Therefore, we apply Fact 4.2.4 to the graphs $L_{v}$ and $\bigcup_{1<i<j \leq k} G^{i j}(v, G)$. Since the size of each partite set $N_{G, j}(v), 1<j \leq k$, of these graphs $L_{v}, \bigcup_{1<i<j \leq k} G^{i j}(v, G)$ satisfies

$$
\left|N_{G, j}(v)\right|>\frac{m}{\lambda}(1-\epsilon)
$$

we apply Fact 4.2 .4 to the graphs $L_{v}$ and $\bigcup_{1<i<j \leq k} G^{i j}(v, G)$ to conclude

$$
\left|\mathcal{K}_{k-1}^{(2)}\left(L_{v}\right)\right| \geq\left(\frac{\alpha}{\lambda}\right)^{\left(\begin{array}{c}
k-1 \\
2
\end{array}\right)}\left(\frac{m}{\lambda}(1-\epsilon)\right)^{k-1}(1-\bar{\beta}) .
$$

We now use (45) to prove the following claim. Recall the collection $\mathcal{S}=\left\{S_{1}, \ldots, S_{q}\right\}$ guaranteed by Claim 5.3.3, where for convenience, we put $S_{1}=\left\{v_{1}, \ldots, v_{r_{2}}\right\}$. The following claim asserts that the number of $(k-1)$-cliques with all edges belonging to a link graph of some vertex $v_{\mu}$ in $S_{1}, 1 \leq \mu \leq r_{2}$, is a large portion of all the $(k-1)$-cliques in the $(k-1)$-partite subgraph of $G$ spanned by the sets $V_{2}, \ldots, V_{k}$.

Claim 5.3.5. Let $B=\{2, \ldots, k\}$ so that $G(B)=\bigcup_{1<i<j \leq k} G^{i j}$. Then

$$
\left|\bigcup_{\mu=1}^{r_{2}} \mathcal{K}_{k-1}^{(2)}\left(L_{v_{\mu}}\right)\right|>\delta\left|\mathcal{K}_{k-1}^{(2)}(G(B))\right| \text {. }
$$

\section{Proof of Claim 5.3.5.}

We apply inclusion-exclusion to $\left|\bigcup_{\mu=1}^{r_{2}} \mathcal{K}_{k-1}^{(2)}\left(L_{v_{\mu}}\right)\right|$ to obtain

$$
\left|\bigcup_{\mu=1}^{r_{2}} \mathcal{K}_{k-1}^{(2)}\left(L_{v_{\mu}}\right)\right| \geq \sum_{\mu=1}^{r_{2}}\left|\mathcal{K}_{k-1}^{(2)}\left(L_{v_{\mu}}\right)\right|-\sum_{\{\mu, \nu\} \in\left[r_{2}\right]^{2}}\left|\mathcal{K}_{k-1}^{(2)}\left(L_{v_{\mu}}\right) \cap \mathcal{K}_{k-1}^{(2)}\left(L_{v_{\nu}}\right)\right| .
$$


To provide an upper bound for the second order term above, we first recall that for all $\{\mu, \nu\} \in\left[r_{2}\right]^{2}$, the vertices $v_{\mu}, v_{\nu} \in S_{1}$ satisfy the property in (44). That property tells us that for all $j, 1<j \leq k$,

$$
\left|N_{G, j}\left(v_{\mu}\right) \cap N_{G, j}\left(v_{\nu}\right)\right| \leq \frac{m}{\lambda^{2}}(1+\epsilon)^{2} .
$$

It follows then from (44) and Fact 2.1.7 that for each $\{\mu, \nu\} \in\left[r_{2}\right]^{2}$,

$$
\begin{aligned}
\left|\mathcal{K}_{k-1}^{(2)}\left(L_{v_{\mu}}\right) \cap \mathcal{K}_{k-1}^{(2)}\left(L_{v_{\nu}}\right)\right| & <\left(1+\theta_{k-1, \lambda}\left(2 \lambda^{2} \epsilon\right)\right) \frac{1}{\lambda^{\left(\begin{array}{c}
k-1 \\
2
\end{array}\right)}}\left(\frac{m}{\lambda^{2}}(1+\epsilon)^{2}\right)^{k-1}, \\
& <\frac{2}{\lambda^{\left(\begin{array}{c}
k-1 \\
2
\end{array}\right)}}\left(\frac{m}{\lambda^{2}}(1+\epsilon)^{2}\right)^{k-1}
\end{aligned}
$$

where the last inequality follows from the fact that we chose $\epsilon$ in (38) and (41) to satisfy $\theta_{k-1, \lambda}\left(2 \lambda^{2} \epsilon\right)<1$. Applying (45) and (48) to the right hand side of (47), we obtain the following further lower bound on $\left|\bigcup_{\mu=1}^{r_{2}} \mathcal{K}_{k-1}^{(2)}\left(L_{v_{\mu}}\right)\right|$ :

$$
\begin{aligned}
\left|\bigcup_{\mu=1}^{r_{2}} \mathcal{K}_{k-1}^{(2)}\left(L_{v_{\mu}}\right)\right| \geq & r_{2}\left(\frac{\alpha}{\lambda}\right)^{\left(\begin{array}{c}
k-1 \\
2
\end{array}\right)}\left(\frac{m}{\lambda}(1-\epsilon)\right)^{k-1}(1-\bar{\beta})- \\
& \left(\begin{array}{c}
r_{2} \\
2
\end{array}\right) \frac{2}{\lambda^{\left(\begin{array}{c}
k-1 \\
2
\end{array}\right)}}\left(\frac{m}{\lambda^{2}}(1+\epsilon)^{2}\right)^{k-1}, \\
& \geq \frac{m^{k-1}}{\lambda^{\left(\begin{array}{c}
k-1 \\
2
\end{array}\right)} P\left(r_{2}\right)}
\end{aligned}
$$

where

$$
P\left(r_{2}\right)=\left[r_{2} \frac{\alpha^{\left(\begin{array}{c}
k-1 \\
2
\end{array}\right)}}{\lambda^{k-1}}(1-\epsilon)^{k-1}(1-\bar{\beta})-r_{2}^{2}\left(\frac{1+\epsilon}{\lambda}\right)^{2(k-1)}\right] .
$$

We now use our value for $r_{2}$ given in (34) and begin by making the following estimations of $r_{2}$.

Note that it trivially follows that $r_{2} \geq \gamma \lambda^{k-1}$. It also trivially follows that

$$
r_{2} \leq \gamma \lambda^{k-1}+1=\gamma \lambda^{k-1}\left(1+\frac{1}{\gamma \lambda^{k-1}}\right)
$$

From our hypothesis that $\lambda \geq \frac{1}{\delta}$, we easily infer that $\delta^{k-1} \geq \frac{1}{\lambda^{k-1}}$. Using this lower bound on $\delta^{k-1}$ in the right hand side of (52), we see

$$
r_{2} \leq \gamma \lambda^{k-1}\left(1+\frac{\delta^{k-1}}{\gamma}\right)
$$

However, it follows from (29) and (31) that $\delta \leq \gamma$, thus we have

$$
\gamma \lambda^{k-1}\left(1+\frac{\delta^{k-1}}{\gamma}\right) \leq \gamma \lambda^{k-1}\left(1+\frac{\delta^{k-2} \gamma}{\gamma}\right) \leq \gamma \lambda^{k-1}(1+\delta)
$$

where the last inequality follows from the fact that $k \geq 3$. In sum,

$$
\gamma \lambda^{k-1} \leq r_{2}=\left\lceil\gamma \lambda^{k-1}\right\rceil \leq \gamma \lambda^{k-1}(1+\delta) .
$$


With $\gamma$ defined in (25), (26), we substitute the bounds on $r_{2}$ given in (53) into the expression in (51) to obtain a further lower bound on $\left|\bigcup_{\mu=1}^{r_{2}} \mathcal{K}_{k-1}^{(2)}\left(L_{v_{\mu}}\right)\right|$. This substitution yields

$$
\left|\bigcup_{\mu=1}^{r_{2}} \mathcal{K}_{k-1}^{(2)}\left(L_{v_{\mu}}\right)\right| \geq \frac{m^{k-1}}{\lambda^{\left(\begin{array}{c}
k-1 \\
2
\end{array}\right)}}\left[\gamma \alpha^{\left(\begin{array}{c}
k-1 \\
2
\end{array}\right)}(1-\epsilon)^{k-1}(1-\bar{\beta})-\gamma^{2}(1+\delta)^{2}(1+\epsilon)^{2(k-1)}\right],
$$

the right hand side of which we equivalently write as

$$
\frac{m^{k-1}}{\lambda^{\left(\begin{array}{c}
k-1 \\
2
\end{array}\right)}} \gamma\left[\alpha^{\left(\begin{array}{c}
k-1 \\
2
\end{array}\right)}(1-\epsilon)^{k-1}(1-\bar{\beta})-\sqrt{\gamma} \sqrt{\gamma}(1+\delta)^{2}(1+\epsilon)^{2(k-1)}\right] .
$$

Recall from (25) that $\gamma$ satisfies

$$
\sqrt{\gamma} \leq \frac{\alpha^{\left(\begin{array}{c}
k-1 \\
2
\end{array}\right)}(1-\bar{\beta})}{4 \cdot 8^{k-1}}
$$

Since $\delta<1$ and $\epsilon<1 / 2$, we easily have that

$$
\sqrt{\gamma} \leq \frac{\alpha^{\left(\begin{array}{c}
k-1 \\
2
\end{array}\right)}(1-\bar{\beta})(1-\epsilon)^{k-1}}{(1+\delta)^{2}(1+\epsilon)^{2(k-1)}} .
$$

Substituting (55) into one of the factors of $\sqrt{\gamma}$ in (54) yields

$$
\left|\bigcup_{\mu=1}^{r_{2}} \mathcal{K}_{k-1}^{(2)}\left(L_{v_{\mu}}\right)\right| \geq \frac{m^{k-1}}{\lambda^{\left(\begin{array}{c}
k-1 \\
2
\end{array}\right)}} \gamma(1-\sqrt{\gamma}) \alpha^{\left(\begin{array}{c}
k-1 \\
2
\end{array}\right)}(1-\bar{\beta})(1-\epsilon)^{k-1} .
$$

Recall that by our choice of $\delta$ in (29) and (31), we have

$$
2 \delta<\frac{\gamma(1-\sqrt{\gamma}) \alpha^{\left(\begin{array}{c}
k-1 \\
2
\end{array}\right)}(1-\bar{\beta})}{2^{k}} .
$$

Since $\epsilon<1 / 2$, we easily have that

$$
2 \delta<\gamma(1-\sqrt{\gamma}) \alpha^{\left(\begin{array}{c}
k-1 \\
2
\end{array}\right)}(1-\bar{\beta})(1-\epsilon)^{k-1} .
$$

Hence, we have from (56) and (57) that

$$
\left|\bigcup_{\mu=1}^{r_{2}} \mathcal{K}_{k-1}^{(2)}\left(L_{v_{\mu}}\right)\right| \geq 2 \delta \frac{m^{k-1}}{\lambda^{\left(\begin{array}{c}
k-1 \\
2
\end{array}\right)}}
$$

On the other hand, it follows from Fact 2.1.7 that

$$
\left|\mathcal{K}_{k-1}^{(2)}(G(B))\right|<\left(1+\theta_{k-1, \lambda}(\epsilon)\right) \frac{m^{k-1}}{\lambda^{\left(\begin{array}{c}
k-1 \\
2
\end{array}\right)}} .
$$

Since $\theta_{k-1, \lambda}(\epsilon)$ decreases as $\epsilon$ decreases, we infer by (38) and (41) that

$$
\theta_{k-1, \lambda}(\epsilon)<\theta_{k-1, \lambda}\left(2 \lambda^{2} \epsilon\right)<1 .
$$

Combining (58), (59), and (60), we infer that

$$
\left|\bigcup_{\mu=1}^{r_{2}} \mathcal{K}_{k-1}^{(2)}\left(L_{v_{\mu}}\right)\right|>\delta\left|\mathcal{K}_{k-1}^{(2)}(G(B))\right| .
$$

Thus Claim 5.3.5 is proved. 
We use Claim 5.3.5 to invoke the $\left(\alpha^{\left(\begin{array}{c}k-1 \\ 3\end{array}\right)}, \delta, r\right)$-regularity of the $(k-1)$-partite 3 -cylinder $\mathcal{H}(B)$ (recall $B=\{2, \ldots, k\})$. Viewing $\left(L_{v_{1}}, \ldots, L_{v_{r_{2}}}\right)$ as an $r_{2}$-tuple of $(k-1)$-partite

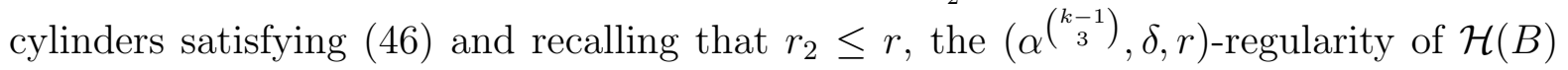
implies

$$
\left|\mathcal{K}_{k-1}^{(3)}(\mathcal{H}(B)) \cap \bigcup_{\mu=1}^{r_{2}} \mathcal{K}_{k-1}^{(2)}\left(L_{v_{\mu}}\right)\right|>\left(\alpha^{\left(\begin{array}{c}
k-1 \\
3
\end{array}\right)}-2 \delta\right)\left|\bigcup_{\mu=1}^{r_{2}} \mathcal{K}_{k-1}^{(2)}\left(L_{v_{\mu}}\right)\right| .
$$

Using the bound in (56) in the right hand side of the above inequality yields

$$
\begin{gathered}
\left|\mathcal{K}_{k-1}^{(3)}(\mathcal{H}(B)) \cap \bigcup_{\mu=1}^{r_{2}} \mathcal{K}_{k-1}^{(2)}\left(L_{v_{\mu}}\right)\right|> \\
\left(\alpha^{\left(\begin{array}{c}
k-1 \\
3
\end{array}\right)}-2 \delta\right) \gamma(1-\sqrt{\gamma}) \alpha^{\left(\begin{array}{c}
k-1 \\
2
\end{array}\right)}(1-\bar{\beta})(1-\epsilon)^{k-1} \frac{m^{k-1}}{\lambda^{\left(\begin{array}{c}
k-1 \\
2
\end{array}\right)}} .
\end{gathered}
$$

In view of (24), (26), (39), and (41), we have

$$
(1-\sqrt{\gamma})(1-\bar{\beta})(1-\epsilon)^{k-1}>(1-\beta)^{\frac{1}{2}},
$$

and also, in view of (27) and (31),

$$
\left(\alpha^{\left(\begin{array}{c}
k-1 \\
3
\end{array}\right)}-2 \delta\right) \alpha^{\left(\begin{array}{c}
k-1 \\
2
\end{array}\right)}=\alpha^{\left(\begin{array}{c}
k \\
3
\end{array}\right)}\left(1-\frac{2 \delta}{\alpha^{\left(\begin{array}{c}
k-1 \\
3
\end{array}\right)}}\right)>\alpha^{\left(\begin{array}{c}
k \\
3
\end{array}\right)}(1-\beta)^{\frac{1}{6}} .
$$

Thus it follows from (61), (62), and (63) that

$$
\left|\mathcal{K}_{k-1}^{(3)}(\mathcal{H}(B)) \cap \bigcup_{\mu=1}^{r_{2}} \mathcal{K}_{k-1}^{(2)}\left(L_{v_{\mu}}\right)\right|>\alpha^{\left(\begin{array}{l}
k \\
3
\end{array}\right)} \gamma(1-\beta)^{\frac{2}{3}} \frac{m^{k-1}}{\lambda^{\left(\begin{array}{c}
k-1 \\
2
\end{array}\right)}} .
$$

We now conclude our argument for the proof of Lemma 5.1.1. It follows from the definition of link graph $L_{v_{\mu}}, 1 \leq \mu \leq r_{2}$, that for all

$$
\left\{w_{2}, \ldots, w_{k}\right\} \in\left(\mathcal{K}_{k-1}^{(3)}(\mathcal{H}(B)) \cap \bigcup_{\mu=1}^{r_{2}} \mathcal{K}_{k-1}^{(2)}\left(L_{v_{\mu}}\right)\right),
$$

the set $\left\{v_{\mu}, w_{2}, \ldots, w_{k}\right\} \in \mathcal{K}_{k}^{(3)}(\mathcal{H})$. Using this fact and repeating the argument for $(64)$ above for all sets $S_{i} \in \mathcal{S}, 1 \leq i \leq q$, we obtain

$$
\begin{aligned}
\left|\mathcal{K}_{k}^{(3)}(\mathcal{H})\right| & \geq \sum_{i=1}^{q}\left|\mathcal{K}_{k-1}^{(3)}(\mathcal{H}(B)) \cap \bigcup_{\mu=1}^{r_{2}} \mathcal{K}_{k-1}^{(2)}\left(L_{v_{\mu}}\right)\right| \\
& >q \alpha^{\left(\begin{array}{c}
k \\
3
\end{array}\right)} \gamma(1-\beta)^{\frac{2}{3}} \frac{m^{k-1}}{\lambda^{\left(\begin{array}{c}
k-1 \\
2
\end{array}\right)}} .
\end{aligned}
$$

Recall $q=\left(1-\frac{(k-1) r_{2} \epsilon}{\sigma}\right) \frac{\sigma m}{r_{2}}$. In light of the fact that $r_{2} \leq \gamma \lambda^{\left(\begin{array}{c}k-1 \\ 2\end{array}\right)}(1+\delta)$, and due to (40) and (43), we have

$$
q=\left(1-\frac{(k-1) r_{2} \epsilon}{\sigma}\right) \frac{\sigma m}{r_{2}} \geq(1-\beta)^{\frac{1}{6}} \frac{\sigma m}{\gamma \lambda^{k-1}(1+\delta)} .
$$


Combining the lower bound for $q$ in (66) with the inequality in (65) implies

$$
\left|\mathcal{K}_{k}^{(3)}(\mathcal{H})\right|>\frac{\sigma}{1+\delta} \alpha^{\left(\begin{array}{l}
k \\
3
\end{array}\right)}(1-\beta)^{\frac{5}{6}} \frac{m^{k}}{\lambda^{\left(\begin{array}{c}
k \\
2
\end{array}\right)}} .
$$

Recall from (43) that $\sigma=1-4 \sqrt{\delta}\left(\begin{array}{c}k-1 \\ 2\end{array}\right)$, and thus

$$
\frac{\sigma}{1+\delta} \geq 1-5 \sqrt{\delta}\left(\begin{array}{c}
k-1 \\
2
\end{array}\right) \text {. }
$$

Recall from our choice of $\delta$ in (28) and (31) that

$$
1-5 \sqrt{\delta}\left(\begin{array}{c}
k-1 \\
2
\end{array}\right)>(1-\beta)^{\frac{1}{6}} .
$$

Using the inequality in (68), we may bound the quantity in the right hand side of (67) further from below to imply

$$
\left|\mathcal{K}_{k}^{(3)}(\mathcal{H})\right|>\frac{\alpha^{\left(\begin{array}{c}
k \\
3
\end{array}\right)}}{\lambda^{\left(\begin{array}{c}
k \\
2
\end{array}\right)}} m^{k}(1-\beta),
$$

thus Lemma 5.1.1 is proved.

\section{The Regularity Lemma}

Our next goal is to prove the implication $D(k) \Rightarrow R(k)$. However, much of our proof of this implication involves the use of a regularity lemma. In this section, we state a regularity lemma for 3-uniform hypergraphs which slightly extends Theorem 3.5 in [2]. The proof of this lemma follows the same lines as that of Theorem 3.5, and we do not include it here. First, we state a number of supporting definitions which are analogous to those found in [2].

\subsection{Definitions for the Regularity Lemma.}

Definition 6.1.1. Let $t$ be an integer and let $V=\bigcup_{i=1}^{k} V_{i},\left|V_{1}\right|=\ldots=\left|V_{k}\right|=N$, be a partition of a $k N$ element set $V$. We define an equitable refinement of $V=\bigcup_{i=1}^{k} V_{i}$ as a partition $V=W=W_{0} \cup \bigcup_{1 \leq i \leq k} \bigcup_{1 \leq x_{i} \leq t} W_{x_{i}}$, where

(i) for any $i \in[k], \bigcup_{1 \leq x_{i} \leq t} W_{x_{i}} \subseteq V_{i}$,

(ii) for each $i, 1 \leq i \leq k$, for each $x_{i}, 1 \leq x_{i} \leq t,\left|W_{x_{i}}\right|=\left\lfloor\frac{N}{t}\right\rfloor=m$,

(iii) $\left|W_{0}\right|<k t$.

Note that $W_{0}=\emptyset$ if $t$ divides $N$. We use

$$
\bigcup_{1 \leq i \leq k} \bigcup_{1 \leq x_{i} \leq t} x_{i}=\left\{1_{1}, \ldots, t_{1}, 2_{1}, \ldots, t_{2}, \ldots, t_{1}, \ldots, t_{k}\right\}
$$

as double-indices.

Definition 6.1.2. Let $k, \lambda, l, t$ be positive integers, $\epsilon, \epsilon_{1}, \epsilon_{2}$ be positive reals. Suppose $G=\bigcup_{1 \leq i<j \leq k} G^{i j}$ is a $(\lambda, \epsilon, k)$-cylinder with $k$-partition $\left(V_{1}, \ldots, V_{k}\right),\left|V_{1}\right|=\ldots=\left|V_{k}\right|=$ $N$. We define a $\left(\lambda l, k t, \epsilon_{1}, \epsilon_{2}\right)$-partition $\mathcal{P}$ of $V(G)=V_{1} \cup \ldots \cup V_{k}$ as a partition $V=$ 
$W=W_{0} \cup \bigcup_{1 \leq i \leq k} \bigcup_{1 \leq x_{i} \leq t} W_{x_{i}}$ of $V(G)$, together with a system of bipartite graphs $P_{\alpha_{x_{i}} y_{j}}^{x_{i} y_{j}}$, $1 \leq i<j \leq k, 1 \leq x_{i}, y_{j} \leq t, 1 \leq \alpha_{x_{i} y_{j}} \leq l_{x_{i} y_{j}} \leq l$, such that:

(0) $W=W_{0} \cup \bigcup_{1 \leq i \leq k} \bigcup_{1 \leq x_{i} \leq t} W_{x_{i}}$ is an equitable refinement of $V=V_{1} \cup \ldots \cup V_{k}$.

(i) For all $i, j, 1 \leq i<j \leq k$, for all $x_{i}, y_{j}, 1 \leq x_{i}, y_{j} \leq t$,

$$
\begin{aligned}
\bigcup_{1 \leq \alpha_{x_{i} y_{j}} \leq l_{x_{i} y_{j}}} P_{\alpha_{x_{i} y_{j}}}^{x_{i} y_{j}} & \subseteq G\left[W_{x_{i}}, W_{y_{j}}\right], \\
& =\left\{\left\{v_{x_{i}}, v_{y_{j}}\right\} \in G: v_{x_{i}} \in W_{x_{i}}, v_{y_{j}} \in W_{y_{j}}\right\} .
\end{aligned}
$$

(ii) All but $\epsilon_{1}\left(\begin{array}{c}k t \\ 2\end{array}\right) m^{2} / \lambda$ pairs $\left\{v_{x_{i}}, v_{y_{j}}\right\} \in G, v_{x_{i}} \in W_{x_{i}}, v_{y_{j}} \in W_{y_{j}}$, are edges of $\frac{\epsilon_{2}}{2 \lambda l^{-}}$ regular bipartite graphs $P_{\alpha_{x_{i} y_{j}}}^{x_{i} y_{j}}, 1 \leq i<j \leq k, 1 \leq x_{i}, y_{j} \leq t, 1 \leq \alpha_{x y} \leq l_{x y} \leq l$, satisfying

$$
\frac{1}{\lambda l}-\frac{\epsilon_{2}}{2 \lambda l}<d_{P_{\alpha_{x_{i}} y_{j}}^{x_{i} y_{j}}}\left(W_{x_{i}}, W_{y_{j}}\right)<\frac{1}{\lambda l}+\frac{\epsilon_{2}}{2 \lambda l} .
$$

Note the following about Definition 6.1.2.

- In statement (0) of Definition 6.1.2, if it is further assumed that $t$ divides $N$, then we have that $W_{0}=\emptyset$, where $W_{0}$ is that residual class of vertices described in Definition 6.1.1.

- Note that since $|G| \sim\left(\begin{array}{c}k \\ 2\end{array}\right) \frac{N^{2}}{\lambda} \leq\left(\begin{array}{c}k t \\ 2\end{array}\right) \frac{m^{2}}{\lambda}$, statement $(i i)$ of Definition 6.1.2 says that all but an $\epsilon_{1}$-portion of the edges of $G$ belong to $\frac{\epsilon_{2}}{2 \lambda l}$-regular graphs $P_{\alpha_{x y}}^{x y}$ of density satisfying (69).

- Note that the $\frac{\epsilon_{2}}{2 \lambda l}$-regular bipartite graphs $P_{\alpha_{x_{i} y_{j}}}^{x_{i} y_{j}}$ each with density satisfying (69) are $\left(\lambda l, \epsilon_{2}, 2\right)$-cylinders. For the remainder of this paper, we refer to such bipartite graphs $P_{\alpha_{x_{i}} y_{j}}^{x_{i} y_{j}}$ specifically as being $\left(\lambda l, \epsilon_{2}, 2\right)$-cylinders.

We now state a definition relating to Definition 2.2.7.

Definition 6.1.3. Let positive integers $k, \lambda, l, t, r$ and positive reals $\delta, \epsilon, \epsilon_{1}, \epsilon_{2}$ be given. Suppose

(i) $G=\bigcup_{1 \leq i<j \leq k} G^{i j}$ is a $(\lambda, \epsilon, k)$-cylinder with $k$-partition $\left(V_{1}, \ldots, V_{k}\right),\left|V_{1}\right|=\ldots=$ $\left|V_{k}\right|=N$.

(ii) $\mathcal{H}$ is a $k$-partite 3 -cylinder with underlying $k$-partite cylinder $G=\bigcup_{1 \leq i<j \leq k} G^{i j}$.

(iii) $\mathcal{P}$ is a $\left(\lambda l, k t, \epsilon_{1}, \epsilon_{2}\right)$-partition of $G$ with equitable refinement $W_{0} \cup \bigcup_{1 \leq i \leq k} \bigcup_{1 \leq x_{i} \leq t} W_{x_{i}}$.

We say that the $\left(\lambda l, k t, \epsilon_{1}, \epsilon_{2}\right)$ - partition $\mathcal{P}$ of $G$ is $(\delta, r)$-regular with respect to $\mathcal{H}$ if

$$
\sum\left\{\left|\mathcal{K}_{3}^{(2)}\left(P_{3}\right)\right|: P_{3} \text { is not a }(\delta, r) \text {-fully regular triad of } \mathcal{P}\right\}<\delta\left(\frac{k N}{\lambda}\right)^{3} \text {. }
$$

Suppose $P_{3}=P_{\alpha}^{x_{h} y_{i}} \cup P_{\beta}^{x_{h} z_{j}} \cup P_{\gamma}^{y_{i} z_{j}}, 1 \leq h<i<j \leq k, \alpha \in\left[l_{x_{h} y_{i}}\right], \beta \in\left[l_{x_{h} z_{j}}\right], \gamma \in\left[l_{y_{i} z_{j}}\right]$, is a triad of the partition $\mathcal{P}$ defined on $W_{x_{h}}, W_{y_{i}}, W_{z_{j}}$. For the triad $P_{3}$, set 


$$
\mu_{P_{3}}=\frac{\left|\mathcal{K}_{3}^{(2)}\left(P_{3}\right)\right|}{m^{3}}
$$

We may equivalently write the inequality in (70) with the notation above as

$$
\sum\left\{\mu_{P_{3}}: P_{3} \text { is not a }(\delta, r) \text {-fully regular triad of } \mathcal{P}\right\}<\delta\left(\frac{k N}{m \lambda}\right)^{3} .
$$

\subsection{Statement of the Regularity Lemma.}

We now state our regularity lemma.

Theorem 6.2.1. For all constants $\epsilon_{1}, \delta$, positive integers $k, \lambda, l_{0}, t_{0}, k \geq 3$, integer valued functions $r(t, l)$, and functions $\epsilon_{2}(l)$, there exist $\epsilon>0$ and integers $T_{0}, L_{0}, N_{0}$ so that if

(i) $G$ is a $(\lambda, \epsilon, k)$-cylinder with $k$ partition $\left(V_{1}, \ldots, V_{k}\right),\left|V_{1}\right|=\ldots=\left|V_{k}\right|=N \geq N_{0}$,

(ii) $\mathcal{H}$ is a k-partite 3-cylinder with $G$ underlying $\mathcal{H}$,

then $\mathcal{H}$ admits a $(\delta, r(t, l))$-regular $\left(\lambda l, k t, \epsilon_{1}, \epsilon_{2}(l)\right)$ partition $\mathcal{P}$, where $l_{0} \leq l \leq L_{0}, t_{0} \leq$ $t \leq T_{0}$.

Note that in Theorem 6.2.1, the numbers $\epsilon, T_{0}, L_{0}, N_{0}$ do not only depend on $\epsilon, \delta, k$, $\lambda, l_{0}$, and $t_{0}$, but also functions $r(t, l)$, and $\epsilon_{2}(l)$.

We mention that there is only one slight difference between Theorem 6.2.1 and the original Theorem 3.5 of Frankl and Rödl in [2]. Indeed, recall in Definition 6.1.2 and Theorem 6.2.1 the $k$-partite graph $G=\bigcup_{1 \leq i<j \leq k} G^{i j}$ had each $G^{i j}, 1 \leq i<j \leq k$, a $(\lambda, \epsilon, 2)$-cylinder. In Theorem 3.5 of [2], essentially speaking, the graph $G$ was $G=K_{k N}$. To be precisely compatible with Definition 6.1.2, Theorem 3.5 of [2] could be formulated with $G=\bigcup_{1 \leq i<j \leq k} K\left[V_{i}, V_{j}\right]$, where $K\left[V_{i}, V_{j}\right]$ denotes the complete bipartite graph on $V_{i} \cup V_{j}, 1 \leq i<j \leq k$. In this way, we see each $K\left[V_{i}, V_{j}\right], 1 \leq i<j \leq k$, is a $(1, \epsilon, 2)$-cylinder and hence the easy generalization from Theorem 3.5 in [2] to Theorem 6.2.1.

It is also possible to prove a slight generalization of Theorem 6.2.1 above. The following statement is analogous to that of Theorem 3.11 in [2], and its proof is also along the same lines as Theorem 3.5 in [2].

Theorem 6.2.2. For all constants $\epsilon_{1}, \delta$, positive integers $s, k, \lambda, l_{0}, t_{0}, k \geq 3$, integer valued functions $r(t, l)$, and functions $\epsilon_{2}(l)$, there exists $\epsilon>0$ and integers $T_{0}, L_{0}, N_{0}$ so that if

(i) $G$ is a $(\lambda, \epsilon, k)$-cylinder with $k$-partition $\left(V_{1}, \ldots, V_{k}\right),\left|V_{1}\right|=\ldots=\left|V_{k}\right|=N \geq N_{0}$,

(ii) $\left\{\mathcal{H}_{1}, \ldots, \mathcal{H}_{s}\right\}$ is any family of $k$-partite 3-cylinders satisfying that for each $i \in[s]$, $G$ underlies $\mathcal{H}_{i}$,

then there exists a $\left(\lambda l, k t, \epsilon_{1}, \epsilon_{2}(l)\right)$ partition $\mathcal{P}$ which is $(\delta, r(t, l))$-regular with respect to each $\mathcal{H}_{i}$, where $l_{0} \leq l \leq L_{0}, t_{0} \leq t \leq T_{0}$.

$$
\text { 7. } D(k) \Longrightarrow R(k)
$$

In this section, we prove the implication $D(k) \Rightarrow R(k)$. Our proof is handled through the upcoming Lemma 7.1.1. 


\subsection{Lemma 7.1.1.}

We recall the familiar Setup 2', and for convenience, we restate this setup. For an integer $k \geq 3, \mathcal{H}$ is as usual a $k$-partite 3 -cylinder, and $G=\bigcup_{1 \leq i<j \leq k} G^{i j}$ is an underlying $k$-partite cylinder of $\mathcal{H}$. In particular, for specified constants $k, \alpha, \delta, \lambda, r, \epsilon$ and $N, \mathcal{H}$ and $G$ satisfy the following Setup:

\section{Setup:}

(i) $\mathcal{H}$ is a $k$-partite 3 -cylinder with $k$-partition $\left(V_{1}, \ldots, V_{k}\right),\left|V_{1}\right|=\ldots=\left|V_{k}\right|=N$,

(ii) $G=\bigcup_{1 \leq i<j \leq k} G^{i j}$ is a $(\lambda, \epsilon, k)$-cylinder underlying $\mathcal{H}$,

(iii) for all $B \in[k]^{3}, \mathcal{H}(B)$ is $(\alpha, \delta, r)$-regular with respect to $G(B)$.

We prove the following lemma.

Lemma 7.1.1. For every integer $k \geq 4$, the statement $D(k)$ implies the statement $R(k)$.

Before giving any formal proofs, we first discuss our strategy for verifying Lemma 7.1.1. For the moment, let us suppose that given constants $k \geq 4, \alpha, \delta_{k}$, a constant $\delta>0$ has been disclosed, and given an integer $\lambda \geq \frac{1}{\delta}$ and an integer $r_{k} \geq 1$, constants $r, \epsilon$, and $N_{0}$ have been disclosed. Suppose 3 -cylinder $\mathcal{H}$ and underlying cylinder $G$ satisfy the conditions of the Setup with the constants $k, \alpha, \delta, \lambda, r, \epsilon$, and $N, N \geq N_{0}$. To say that $\mathcal{H}$ is $\left(\alpha^{\left(\begin{array}{c}k \\ 3\end{array}\right)}, \delta_{k}, r_{k}\right)$-regular with respect to $G$ means the following (cf., Definition 2.2.6): let $\vec{Q}=(Q(s)), 1 \leq s \leq r_{k}$, be an $r_{k}$-tuple, where for all $s \in\left[r_{k}\right], Q(s)=\bigcup_{1 \leq i<j \leq k} Q^{i j}(s)$, and for all $\{i, j\} \in[k]^{2}, s \in\left[r_{k}\right], Q^{i j}(s) \subseteq G^{i j}$. If $\vec{Q}$ satisfies

$$
\left|\bigcup_{s=1}^{r_{k}} \mathcal{K}_{k}^{(2)}(Q(s))\right|>\delta_{k}\left|\mathcal{K}_{k}^{(2)}(G)\right|
$$

then $\vec{Q}$ also satisfies $d_{\mathcal{H}}(\vec{Q})>\alpha^{\left(\begin{array}{c}k \\ 3\end{array}\right)}-2 \delta_{k}$, or equivalently,

$$
\left|\mathcal{K}_{k}^{(3)}(\mathcal{H}) \cap \bigcup_{s=1}^{r_{k}} \mathcal{K}_{k}^{(2)}(Q(s))\right|>\left(\alpha^{\left(\begin{array}{c}
k \\
3
\end{array}\right)}-2 \delta_{k}\right)\left|\bigcup_{s=1}^{r_{k}} \mathcal{K}_{k}^{(2)}(Q(s))\right| .
$$

Therefore, to prove Lemma 7.1.1, we need only show that for any such $\vec{Q}$, the inequality in (72) is indeed satisfied.

While the proof of Lemma 7.1.1 is complicated in its technical details, the idea behind it is simple. We now give an outline for the proof of Lemma 7.1.1 for the case that $r_{k}=1$. Suppose that 3-cylinder $\mathcal{H}$ and underlying cylinder $G$ satisfy the conditions of the Setup with constants $k, \alpha, \delta, \lambda, r, \epsilon$ and $N$. Suppose $Q=\bigcup_{1 \leq i<j \leq k} Q^{i j}$ is given so that for all $\{i, j\} \in[k]^{2}, Q^{i j} \subseteq G^{i j}$, and $\left.\mid \mathcal{K}_{k}^{(2)}(Q)\right)\left|>\delta_{k}\right| \mathcal{K}_{k}^{(2)}(G) \mid$. We show

$$
\left|\mathcal{K}_{k}^{(3)}(\mathcal{H}) \cap \mathcal{K}_{k}^{(2)}(Q)\right|>\left(\alpha^{\left(\begin{array}{l}
k \\
3
\end{array}\right)}-2 \delta_{k}\right)\left|\bigcup_{s=1}^{r_{k}} \mathcal{K}_{k}^{(2)}(Q(s))\right| .
$$

We begin by restricting the hypergraph $\mathcal{H}$ to $\mathcal{H}^{Q}=\mathcal{H} \cap \mathcal{K}_{3}^{(2)}(Q)$. We use our Regularity Lemma, Theorem 6.2.1, to obtain a $\left(\lambda l, k t, \epsilon_{1}, \epsilon_{2}(l)\right)$ partition $\mathcal{P}_{0}$ of the hypergraph $\mathcal{H}^{Q}$ which is $(\delta, r(t, l))$-regular with respect to underlying cylinder $G$, where $W=W_{0} \cup$ $\bigcup_{1 \leq i \leq k} \bigcup_{1 \leq x_{i} \leq t} W_{x_{i}}$ is the equitable refinement of $V=V_{1} \cup \ldots \cup V_{k}$ associated with $\mathcal{P}_{0}$, and $P_{\alpha_{x_{i} y_{j}}}^{x_{i} y_{j}}, 1 \leq i<j \leq k, 1 \leq x_{i}, y_{j} \leq t, 1 \leq \alpha_{x_{i} y_{j}} \leq l_{x_{i} y_{j}} \leq l$ is the system of bipartite 
graphs associated with $\mathcal{P}_{0}$. We consider $k$-partite small cylinders $C_{k}=\bigcup_{\left\{x_{i}, y_{j}\right\} \in[B]^{2}} P_{\alpha_{x_{i} y_{j}}}^{x_{i} y_{j}}$ within the partition $\mathcal{P}_{0}$, where the sets $B$ satisfy $B \subseteq \bigcup_{1 \leq i \leq k} \bigcup_{1 \leq x_{i} \leq t} x_{i},|B|=k$, and for each pair $\left\{x_{i}, y_{j}\right\} \in[B]^{2}, i \neq j$. Our goal is to apply the statement $D(k)$ to "appropriate" small $k$-partite cylinders $C_{k}$. To that end, we characterize a class of $k$-partite small cylinders $C_{k}$, the class $\Pi_{k}$ of perfect cylinders. Perfect cylinders are those small $k$-partite cylinders $C_{k}$ for which the statement $D(k)$ applies (i.e. all triads of $C_{k}$ are "dense and regular", and all bipartite graphs $P_{\alpha_{x_{i} y_{j}}}^{x_{i} y_{j}}$ of $C_{k}$ are "dense and regular"). We show that very few copies of $K_{k}^{(2)}$ from $\mathcal{K}_{k}^{(2)}(Q)$ belong to non perfect cylinders (we call these cylinders defective cylinders). Since nearly all copies of $K_{k}^{(2)}$ from $\mathcal{K}_{k}^{(2)}(Q)$ belong to perfect cylinders $C_{k} \in \Pi_{k}$, we conclude a lower bound on the number of perfect cylinders $\left|\Pi_{k}\right|$. We apply the statement $D(k)$ to each perfect cylinder $C_{k} \in \Pi_{k}$ to conclude a lower bound on $\left|\mathcal{K}_{k}^{(3)}\left(\mathcal{H}^{Q}\right) \cap \mathcal{K}_{k}^{(2)}\left(C_{k}\right)\right|$. We then sum this number over all such perfect $C_{k}$ (we can estimate $\left.\left|\Pi_{k}\right|\right)$ to obtain the inequality in (73).

However, in order to make our plan precise, we need to disclose the constants promised by Lemma 7.1.1. In what follows, we define these constants and then return to prove (72) in the upcoming Proposition 7.2.4.

\subsection{Proving Lemma 7.1.1. .}

We now begin with the definitions of the constants involved in Lemma 7.1.1. However, the Reader may feel free to skip through the tedious definitions and observe that, similarly to Lemma 5.1.1, we commit ourselves to the following hierarchy

$$
\alpha, \delta_{k} \gg \delta>\frac{1}{\lambda} \gg \frac{1}{r} \gg \epsilon \gg \frac{1}{N_{0}}
$$

We mention that it is necessary for

$$
r \geq r_{k}
$$

We explain the reason for this (subtle) requirement later in context.

\section{Definitions of the Constants in Lemma 7.1.1}

In order to start with the formal definitions, observe that the statement $R(k)$ compactly stated says " $\forall \alpha, \delta_{k}, \exists \delta: \forall \lambda \geq \frac{1}{\delta}, \forall r_{k} \geq 1, \exists r, \epsilon, N_{0}$ so that ...". Let $k \geq 4$, and let $\alpha, \delta_{k}$ be two given positive reals. Note that we may assume that $2 \delta_{k}<\alpha\left(\begin{array}{l}k \\ 3\end{array}\right)$, since otherwise Lemma 7.1.1 would be trivial. With $\alpha$, and $\delta_{k}$ given, we first define the promised constant $\delta$.

\section{Definition of $\delta$}

In the definition of $\delta$, we invoke the statement $D(k)$, which recall compactly stated says $" \forall \alpha, \beta, \exists \delta_{D(k)}=\delta_{D(k)}(\alpha, \beta): \forall \lambda \geq \frac{1}{\delta}, \exists r_{D(k)}=r_{D(k)}(\alpha, \beta, \delta, \lambda), \epsilon_{D(k)}=\epsilon_{D(k)}(\alpha, \beta, \delta, \lambda)$ so that ...". Apply the parameters $\frac{\alpha}{2}$, and $\beta=\delta_{k}$ to the statement $D(k)$, where $\alpha, \delta_{k}, k$ are fixed above. Let

$$
\delta_{D(k)}=\delta_{D(k)}\left(\frac{\alpha}{2}, \delta_{k}\right)
$$


be that value guaranteed by statement $D(k)$. Set $\delta>0$ to be small enough so that each of the following inequalities are satisfied:

$$
\begin{aligned}
& \delta<\frac{\alpha}{4}, \\
& \delta \leq \delta_{D(K)},
\end{aligned}
$$

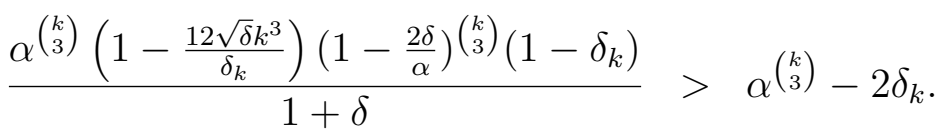

Thus, we have defined the promised constant $\delta$. Note that with $\delta$ fixed, it follows that

$$
\delta \leq \delta_{D(k)}\left(\frac{\alpha}{2}, \delta_{k}\right) \leq \delta_{D(k)}\left(\alpha-2 \delta, \delta_{k}\right) .
$$

Let $\lambda \geq \frac{1}{\delta}, r_{k} \geq 1$ be given integers. Our next goal is to produce the promised constants $r, \epsilon$, and $N_{0}$.

\section{Definitions of $\mathbf{r}, \epsilon$, and $\mathrm{N}_{0}$}

The definitions of the constants $r, \epsilon$, and $N_{0}$ depend on the output of two previous statements, the statement $D(k)$ and our Regularity Theorem, Theorem 6.2.1. As a result, we are only momentarily able to define these constants (cf., (97) for the definition of $r$, (90) for the definition of $\epsilon$ and (93) for the definition of $N_{0}$ ).

First, we consider the statement $D(k)$. Recall that with $\alpha$ and $\delta_{k}$ given, we have defined $\delta$ and subsequently have $\lambda \geq \frac{1}{\delta}$ and $r_{k} \geq 1$ arbitrarily given. Let $l$ be an integer variable. For the parameters $\alpha-2 \delta, \beta=\delta_{k}, \delta, \lambda l$ let

$$
\begin{aligned}
& r_{D(k)}(l)=r_{D(k)}\left(\alpha-2 \delta, \delta_{k}, \delta, \lambda l\right), \\
& \epsilon_{D(k)}(l)=\epsilon_{D(k)}\left(\alpha-2 \delta, \delta_{k}, \delta, \lambda l\right)
\end{aligned}
$$

be guaranteed by the statement $D(k)$. We specify the value $l$ seen in (80) and (81) only after an application of the Regularity Theorem, Theorem 6.2.1.

Recall the Regularity Theorem, as it is stated in Section 6. We may more compactly write Theorem 6.2 .1 as the following " $\forall \epsilon_{1}, \delta, \forall k, \lambda, l_{0}, t_{0}: k \geq 3, \forall r(t, l), \epsilon_{2}(l)$, $\exists \epsilon, T_{0}, L_{0}, N_{0}$, so that ...". We now describe the input for Theorem 6.2.1. Let $\delta$ be given in (76)-(78), let $k \geq 4$ be given in the beginning of the Definitions of the Constants in Lemma 7.1.1, and let $\lambda \geq \frac{1}{\delta}$ be given as above. Since $\delta, k$, and $\lambda$ were already chosen, we now define the remaining constants:

$$
\begin{aligned}
\epsilon_{1} & =\frac{\sqrt{\delta}}{\lambda^{(k}\left(\begin{array}{l}
k \\
2
\end{array}\right)}, \\
l_{0} & =\left\lceil\frac{1}{\delta}\right\rceil, \\
t_{0} & =1 .
\end{aligned}
$$

Now we must produce the input functions $r(t, l)$ and $\epsilon_{2}(l)$. Recall the definition of $r_{D(k)}(l)$ in (80). For arbitrary positive integers $l$ and $t$, set

$$
r(t, l)=\max \left\{2 r_{k} t^{3} l^{3}, r_{D(k)}(l)\right\} .
$$


Recall Fact 2.1.7. For the definition of the function $\epsilon_{2}(l)$, we need this fact, as well as the following two consequences of it. We state these easy consequences of Fact 2.1.7 without proof.

Fact 7.2.1. For any positive integers $k, \lambda$, and suitably small positive reals $\epsilon$, there exists a function $\theta_{k, \lambda}^{\prime}(\epsilon), \theta_{k, \lambda}^{\prime}(\epsilon) \rightarrow 0$ as $\epsilon \rightarrow 0$, such that whenever $G$ is a $(\lambda, \epsilon, k)$-cylinder with $k$-partition $\left(V_{1}, \ldots, V_{k}\right),\left|V_{1}\right|=\ldots=\left|V_{k}\right|=m$, the following holds.

For each $i, j, 1 \leq i<j \leq k$, all but $2 k \epsilon m^{2}$ edges $e=\left\{v_{i}, v_{j}\right\} \in G, v_{i} \in V_{i}$, $v_{j} \in V_{j}$, satisfy

$$
\left(1-\theta_{k, \lambda}^{\prime}(\epsilon)\right) \frac{m^{k-2}}{\lambda^{\left(\begin{array}{c}
k \\
2
\end{array}\right)-1}}<\left|\left\{Y \in \mathcal{K}_{k}^{(2)}(G): v_{i}, v_{j} \in Y\right\}\right|<\left(1+\theta_{k, \lambda}^{\prime}(\epsilon)\right) \frac{m^{k-2}}{\lambda^{\left(\begin{array}{l}
k \\
2
\end{array}\right)-1}} .
$$

Fact 7.2.2. For any positive integers $k, \lambda$, and suitably small positive reals $\epsilon$, there exists a function $\theta_{k, \lambda}^{\prime \prime}(\epsilon), \theta_{k, \lambda}^{\prime \prime}(\epsilon) \rightarrow 0$ as $\epsilon \rightarrow 0$, such that whenever $G$ is a $(\lambda, \epsilon, k)$-cylinder with $k$-partition $\left(V_{1}, \ldots, V_{k}\right),\left|V_{1}\right|=\ldots=\left|V_{k}\right|=m$, the following holds.

For each $i_{1}, i_{2}, i_{3}, 1 \leq i_{1}<i_{2}<i_{3} \leq k$, all but $6 k \epsilon m^{3}$ triangles $t=$

$$
\left\{v_{i_{1}}, v_{i_{2}}, v_{i_{3}}\right\} \in \mathcal{K}_{3}^{(2)}(G), v_{i_{1}} \in V_{i_{1}}, v_{i_{2}} \in V_{i_{2}}, v_{i_{3}} \in V_{i_{3}} \text { satisfy }
$$

$$
\begin{aligned}
\left(1-\theta_{k, \lambda}^{\prime \prime}(\epsilon)\right) \frac{m^{k-3}}{\lambda\left(\begin{array}{c}
k \\
2
\end{array}\right)-3} & <\left|\left\{Y \in \mathcal{K}_{k}^{(2)}(G): v_{i_{1}}, v_{i_{2}}, v_{i_{3}} \in Y\right\}\right| \\
& <\left(1+\theta_{k, \lambda}^{\prime \prime}(\epsilon)\right) \frac{m^{k-3}}{\lambda^{\left(\begin{array}{c}
k \\
2
\end{array}\right)-3}}
\end{aligned}
$$

The definition of the function $\epsilon_{2}(l)$ depends on functions $\theta_{k, \lambda l}(\epsilon), \theta_{k, \lambda l}^{\prime}(\epsilon), \theta_{k, \lambda l}^{\prime \prime}(\epsilon)$ defined in Facts 2.1.7, 7.2.1, 7.2.2. Note that these functions all tend to zero as their argument $\epsilon$ tends to zero. For an arbitrary positive integer $l$, let $\epsilon_{2}(l)$ be a positive quantity satisfying each of the following inequalities

$$
\begin{aligned}
\epsilon_{2}(l) & \leq \epsilon_{D(k)}(l), \\
\theta_{3, \lambda l}\left(\epsilon_{2}(l)\right) & <\frac{1}{10}, \\
\theta_{k, \lambda l}\left(\epsilon_{2}(l)\right), \theta_{k, \lambda l}^{\prime}\left(\epsilon_{2}(l)\right), \theta_{k, \lambda l}^{\prime \prime}\left(\epsilon_{2}(l)\right) & <1 / 2, \\
\theta_{k, \lambda l}\left(\epsilon_{2}(l)\right) & <\delta .
\end{aligned}
$$

For the input values $\epsilon_{1}, \delta, \lambda, l_{0}, t_{0}, r(t, l)$, and $\epsilon_{2}(l)$ given above, let

$$
\begin{aligned}
\epsilon & =\epsilon\left(\epsilon_{1}, \delta, \lambda, l_{0}, t_{0}, r(t, l), \epsilon_{2}(l)\right), \\
T_{0} & =T_{0}\left(\epsilon_{1}, \delta, \lambda, l_{0}, t_{0}, r(t, l), \epsilon_{2}(l)\right), \\
L_{0} & =L_{0}\left(\epsilon_{1}, \delta, \lambda, l_{0}, t_{0}, r(t, l), \epsilon_{2}(l)\right), \\
N_{0} & =N_{0}\left(\epsilon_{1}, \delta, \lambda, l_{0}, t_{0}, r(t, l), \epsilon_{2}(l)\right)
\end{aligned}
$$


be the constants guaranteed by Theorem 6.2.1. Note that we may assume without loss of generality that $\epsilon$ from (90) satisfies

$$
\begin{aligned}
\epsilon & <\frac{\sqrt{\delta}}{6 k \lambda\left(\begin{array}{l}
k \\
2
\end{array}\right)}, \\
\theta_{k, \lambda}(\epsilon), \theta_{k, \lambda}^{\prime}(\epsilon), \theta_{k, \lambda}^{\prime \prime}(\epsilon) & <\frac{1}{2}, \\
\theta_{3, \lambda}(\epsilon) & <\frac{1}{2} .
\end{aligned}
$$

We stress here that the values $\epsilon, T_{0}, L_{0}$, and $N_{0}$ are now fixed constants. We define the constant $r$ as

$$
r=r\left(T_{0}, L_{0}\right)
$$

where the function $r(t, l)$ is given in (85). Having defined the constants above, we now proceed to the proof of Lemma 7.1.1.

\section{Proof of Lemma 7.1.1.}

Given $\alpha$ and $\delta_{k}$, let $\delta$ be given in (76), (77) and (78). Given integers $\lambda \geq \frac{1}{\delta}$ and $r_{k} \geq 1$ let $r, \epsilon$, and $N_{0}$ be given in (97), (90), and (93) respectively, and let $N \geq N_{0}$. We may think of the constants satisfying the following hierarchy:

$$
\alpha \gg \delta_{k} \gg \delta>\frac{1}{\lambda} \gg \frac{1}{r} \gg \epsilon .
$$

We make the following remark describing a small simplifying assumption we make in our proof.

Remark 7.2.3. To simplify some of the details in this proof, we assume that $\left(T_{0}\right)$ ! divides $N$, where $T_{0}$ is given in (91). This assumption is not essential for the proof of Lemma 7.1.1, but it is more convenient to assume it. We make further notes on this assumption later in context. This concludes our remark.

Let $\mathcal{H}$ be a $k$-partite 3 -cylinder and $G$ a $k$-partite underlying cylinder of $\mathcal{H}$ satisfying the conditions of the Setup in the beginning of the section with the constants $k, \alpha, \delta, \lambda$, $r, \epsilon$ and $N$. That is, suppose

(i) $\mathcal{H}$ is a $k$-partite 3 -cylinder with $k$-partition $\left(V_{1}, \ldots, V_{k}\right),\left|V_{1}\right|=\ldots=\left|V_{k}\right|=N$,

(ii) $G=\bigcup_{1 \leq i<j \leq k} G^{i j}$ is a $(\lambda, \epsilon, k)$-cylinder underlying $\mathcal{H}$,

(iii) for all $B \in[k]^{3}, \mathcal{H}(B)$ is $(\alpha, \delta, r)$-regular with respect to $G(B)$.

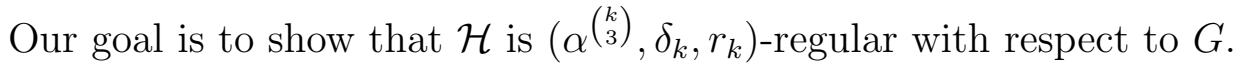

Recall that directly after the statement of Lemma 7.1.1, we discussed what it meant for $\mathcal{H}$ to be $\left(\alpha^{\left(\begin{array}{c}k \\ 3\end{array}\right)}, \delta_{k}, r_{k}\right)$-regular with respect to $G$; we said we need only show the inequality in (72). More precisely, suppose $\vec{Q}=(Q(s)), 1 \leq s \leq r_{k}$, is an $r_{k}$-tuple, where for all $s \in\left[r_{k}\right], Q(s)=\bigcup_{1 \leq i<j \leq k} Q^{i j}(s)$, and for all $\{i, j\} \in[k]^{2}, s \in\left[r_{k}\right], Q^{i j}(s) \subseteq G^{i j}$. We show 
that if

$$
\left|\bigcup_{s=1}^{r_{k}} \mathcal{K}_{k}^{(2)}(Q(s))\right|>\delta_{k}\left|\mathcal{K}_{k}^{(2)}(G)\right|
$$

then

$$
d_{\mathcal{H}}(\vec{Q})>\alpha^{\left(\begin{array}{l}
k \\
3
\end{array}\right)}-2 \delta_{k}
$$

or equivalently,

$$
\left|\mathcal{K}_{k}^{(3)}(\mathcal{H}) \cap \bigcup_{s=1}^{r_{k}} \mathcal{K}_{k}^{(2)}(Q(s))\right|>\left(\alpha^{\left(\begin{array}{l}
k \\
3
\end{array}\right)}-2 \delta_{k}\right)\left|\bigcup_{s=1}^{r_{k}} \mathcal{K}_{k}^{(2)}(Q(s))\right| .
$$

To this effect, assume $\vec{Q}=(Q(s)), 1 \leq s \leq r_{k}$, where for all $s \in\left[r_{k}\right], Q(s)=$ $\bigcup_{1 \leq i<j \leq k} Q^{i j}(s)$, and for all $\{i, j\} \in[k]^{2}, s \in\left[r_{k}\right], Q^{i j}(s) \subseteq G^{i j}$. Assume further that $\vec{Q}$ satisfies (99). Thus, in view of the discussion above, we will have proved Lemma 7.1.1 once we have established (100). This is the subject of the following Proposition 7.2.4.

\section{Proposition 7.2.4.}

$$
\left|\mathcal{K}_{k}^{(3)}(\mathcal{H}) \cap \bigcup_{s=1}^{r_{k}} \mathcal{K}_{k}^{(2)}(Q(s))\right|>\left(\alpha^{\left(\begin{array}{l}
k \\
3
\end{array}\right)}-2 \delta_{k}\right)\left|\bigcup_{s=1}^{r_{k}} \mathcal{K}_{k}^{(2)}(Q(s))\right| .
$$

\subsection{Itinerary for the Proof of Proposition 7.2.4.}

The proof of this proposition depends largely on one single fact which we prove in the upcoming Lemma 7.3.14. However, we will not be able to state Lemma 7.3.14 until we establish some definitions and notation. Therefore, the following material is used to describe the situation discussed in Lemma 7.3.14. We break this material into the following parts.

A. Applying the Regularity Lemma: Here we apply Theorem 6.2.1 to the hypergraph $\mathcal{H}^{Q}=\mathcal{H} \cap \bigcup_{s=1}^{r_{k}} \mathcal{K}_{3}^{(2)}(Q(s))$ with underlying cylinder $G$ to obtain a $\left(\lambda l, k t, \epsilon_{1}, \epsilon_{2}\right)$-partition $\mathcal{P}_{0}$. Note that as a consequence of this application, we disclose specific values of $l$ and $t, l_{0} \leq l \leq L_{0}, t_{0} \leq t \leq T_{0}$, where $l_{0}, L_{0}, t_{0}$ and $T_{0}$ are given in (83), (92), (84) and (91) respectively.

B. Big and Small Cylinders: Here we define structures from the partition $\mathcal{P}_{0}$ over which we work.

C. Defective and Perfect Cylinders: Here we define special classes of small cylinders.

When we fill in the above outline with details, we then state Lemma 7.3.14. After stating Lemma 7.3.14, we proceed directly to the proof of Proposition 7.2.4 in Subsection 7.4. After concluding the proof of Proposition 7.2.4, we begin the task of proving Lemma 7.3.14 in Subsection 7.5.

To begin, restrict $\mathcal{H}$ to the $k$-partite 3 -cylinder $\mathcal{H}^{Q}$ defined by

$$
\mathcal{H}^{Q}=\mathcal{H} \cap \bigcup_{s=1}^{r_{k}} \mathcal{K}_{3}^{(2)}(Q(s)) \subset \mathcal{H} .
$$

Clearly, these are the triples of $\mathcal{H}$ with which (100) is concerned. 
We make the following remark about the hypergraph $\mathcal{H}^{Q}$ with respect to the requirement we mentioned in (74).

Remark 7.3.1. We note that here we use the fact that $r \geq r_{k}$ specified in (74). It is this condition which guarantees that the 3 -cylinder $\mathcal{H}^{Q}$ is nonempty. Indeed, we are given an $r_{k}$-tuple $\vec{Q}=(Q(s))$, satisfying (99). It is not hard to show that for any $B \in[k]^{3}$, $\left|\left(\bigcup_{s=1}^{r_{k}} \mathcal{K}_{3}^{(2)}(Q(s))\right) \cap \mathcal{K}_{3}^{(2)}(G(B))\right|>\delta\left|\mathcal{K}_{3}^{(2)}(G(B))\right|$. Therefore, with $r \geq r_{k}$, it follows from the $(\alpha, \delta, r)$-regularity of the triad $G(B)$ that $\left|\mathcal{H}^{Q}(B)\right|=\left|\mathcal{H}(B) \cap \bigcup_{s=1}^{r_{k}} \mathcal{K}_{3}^{(2)}(Q(s))\right|>$ $(\alpha-2 \delta)\left|\left(\bigcup_{s=1}^{r_{k}} \mathcal{K}_{3}^{(2)}(Q(s))\right) \cap \mathcal{K}_{3}^{(2)}(G(B))\right|>(\alpha-2 \delta) \delta\left|\mathcal{K}_{3}^{(2)}(G(B))\right|>\frac{(\alpha-2 \delta) \delta}{2} \frac{N^{3}}{\lambda^{3}}$ where the last inequality follows from the fact that $G(B)$ is a $(\lambda, \epsilon, 3)$-cylinder and since in Fact 2.1.7, we have that $\theta_{3, \lambda}(\epsilon)<\frac{1}{2}$ from (96). Provided that $N$ is sufficiently large, we thus conclude that $\left|\mathcal{H}^{Q}(B)\right|$ is positive for all $B \in[k]^{3}$. This concludes our remark.

\section{A. Applying the Regularity Theorem:}

We now appeal to the Regularity Lemma, Theorem 6.2.1. We apply Theorem 6.2.1 to the $k$-partite 3 -cylinder $\mathcal{H}^{Q}$ given above with the underlying $(\lambda, \epsilon, k)$-cylinder $\bigcup_{1 \leq i<j \leq k} G^{i j}$. Using parameters

$$
\epsilon_{1}, \delta, \lambda, l_{0}, t_{0}, r(t, l), \epsilon_{2}(l)
$$

given by (82)-(89) and $\lambda \geq \frac{1}{\delta}$ given at the beginning of the Definitions of the Constants, Theorem 6.2.1 guarantees constants

$$
\epsilon, L_{0}, T_{0}, N_{0}
$$

which were disclosed in (90)-(93) in the Definitions of the Constants. For $\mathcal{H}^{Q}$ and $G$ defined above, the application of the Regularity Lemma also gives a $\left(\lambda l, k t, \epsilon_{1}, \epsilon_{2}(l)\right)$ partition $\mathcal{P}_{0}$ which is $(\delta, r(t, l))$-fully regular with respect to $\mathcal{H}^{Q}$, where $l_{0} \leq l \leq L_{0}, t_{0} \leq$ $t \leq T_{0}$. We emphasize to the Reader the following subtle point. All the parameters seen in (102) and (103) were already defined in the Definitions of the Constants. Moreover, they did not depend on the choice of the hypergraph $\mathcal{H}^{Q}$, nor did they depend on the choice of graph $G$, so they could be disclosed without reference to specific hypergraphs or graphs. Now that we have a specific hypergraph $\mathcal{H}^{Q}$ and underlying graph $G$, we also obtain a concrete partition $\mathcal{P}_{0}$ of the specific hypergraph $\mathcal{H}^{Q}$ and $G$. Therefore, the only thing new here is the partition $\mathcal{P}_{0}$, and specific constant values $l$ and $t$ satisfying $l_{0} \leq l \leq L_{0}$ and $t_{0} \leq t \leq T_{0}$. Since after the application of Theorem 6.2.1 above, the values $l, t$ become fixed constants, consequently, $r(t, l), \epsilon_{2}(l)$, are fixed constants. We set $r_{1}=r(t, l)$, recall from $(97)$ that we set $r=r\left(T_{0}, L_{0}\right)$, and observe that

$$
r_{k} \leq r_{1} \leq r
$$

holds. We also set $\epsilon_{2}=\epsilon_{2}(l)$.

Now we are able to summarize all the constants used in Section 7. Together with previously defined constants, we note that our set of fixed constants now consists of

$$
k, \alpha, \delta_{k}, \delta, \lambda, r_{k}
$$


given at the beginning of the Definitions of the Constants (specifically, $\delta$ given in (77)$(78))$

$$
\epsilon_{1}, l_{0}, t_{0}
$$

given as input for the Regularity Lemma in (82)-(84),

$$
\epsilon, L_{0}, T_{0}, N_{0}, l, t
$$

given as output of the Regularity Lemma in (90)-(93) ( $l$ and $t$ just obtained in the most recent application of the Regularity Lemma), and consequently

$$
r, r_{1}, \epsilon_{2}
$$

given by (97), $r_{1}=r(t, l)$, and $\epsilon_{2}=\epsilon_{2}(l)$. We think of these constants satisfying the following hierarchy

$$
\alpha \gg \delta_{k} \gg \delta>\frac{1}{\lambda} \gg \epsilon_{1}, \frac{1}{(l \lambda)^{k}} \gg \epsilon_{2} \gg \frac{1}{T_{0}} \gg \epsilon \gg \frac{1}{N}
$$

Note that the hierarchy above updates the one given in (98).

Throughout the remainder of Section 7 , we denote by $\mathcal{P}_{0}$ that $\left(\lambda l, k t, \epsilon_{1}, \epsilon_{2}\right)$-partition which is $\left(\delta, r_{1}\right)$-fully regular with respect to $\mathcal{H}^{Q}$. We use the following notation to characterize $\mathcal{P}_{0}$.

Let $W_{x_{i}}, 1 \leq i \leq k, 1 \leq x_{i} \leq t$ denote the vertex classes of $\mathcal{P}_{0}$ so that $W=$ $\bigcup_{1 \leq i \leq k} \bigcup_{1 \leq x_{i} \leq t} W_{x_{i}}$ is an equitable refinement of $V=V_{1} \cup \ldots \cup V_{k}$, that is, so that:

(i) For all $i, 1 \leq i \leq k$, and all $x_{i}, 1 \leq x_{i} \leq t,\left|W_{x_{i}}\right|=m_{\mathcal{P}_{0}}=m$.

(ii) For all $i \in[k], \bigcup_{1 \leq x_{i} \leq t} W_{x_{i}}=V_{i}$.

Note that it follows from our assumption that $\left(T_{0}\right)$ ! divides $N$ that $m t=N$ holds. Hence, we now see that this auxiliary divisibility assumption made above was so that the garbage class of vertices $W_{0}$ (cf., Definition 6.1.1) would satisfy $W_{0}=\emptyset$.

Further, we let $P_{\alpha_{x_{i} y_{j}}}^{x_{i} y_{j}}, 1 \leq i \leq k, 1 \leq x_{i}, y_{j} \leq t, 1 \leq \alpha_{x_{i} y_{j}} \leq l_{x_{i} y_{j}} \leq l$, denote the system of bipartite graphs of $\mathcal{P}_{0}$ such that:

(iii) For all $i, j, 1 \leq i<j \leq k$, for all $x_{i}, y_{j}, 1 \leq x_{i}, y_{j} \leq t$,

$$
\begin{aligned}
\bigcup_{1 \leq \alpha_{x_{i} y_{j}} \leq l_{x_{i} y_{j}} \leq l} P_{\alpha_{x_{i} y_{j}}}^{x_{i} y_{j}} & \subseteq G\left[W_{x_{i}}, W_{y_{j}}\right] \\
& =\left\{\left\{v_{x_{i}}, v_{y_{j}}\right\} \in G: v_{x_{i}} \in W_{x_{i}}, v_{y_{j}} \in W_{y_{j}}\right\} .
\end{aligned}
$$

(iv) All but $\epsilon_{1} t^{2}\left(\begin{array}{l}k \\ 2\end{array}\right) m^{2} / \lambda$ pairs $\left\{v_{x_{i}}, v_{y_{j}}\right\} \in G, v_{x_{i}} \in W_{x_{i}}, v_{y_{j}} \in W_{y_{j}}$, are edges of $\left(\lambda l, \epsilon_{2}, 2\right)$-cylinders $P_{\alpha_{x_{i} y_{j}}}^{x_{i} y_{j}}, 1 \leq i<j \leq k, 1 \leq x_{i}, y_{j} \leq t, 1 \leq \alpha_{x_{i} y_{j}} \leq l_{x_{i} y_{j}} \leq l$.

We now proceed to Part B. Before doing so, we make the following brief comment. Note that Lemma 7.1.1 is used to show the implication $D(k) \Longrightarrow R(k)$, which is a statement for $k \geq 4$, and we fixed $k \geq 4$ in the Definitions of the Constants. It is this reason why in what follows, we distinguish between triads and $k$-partite cylinders.

\section{B. Big and Small Cylinders}


Definition 7.3.2. For $B \subseteq[k],|B|=b$, we refer to the $B$-cylinder $G(B)=\bigcup_{\{i, j\} \in[B]^{2}} G^{i j}$ as the big $B$-cylinder, or more generally, as a $b$-partite big cylinder. In the frequently occurring case that $b=3$, we refer to this cylinder as a big triad.

Analogous to Definition 7.3.2, we define "small" cylinders with partite sets $W_{x_{i}}, 1 \leq$ $i \leq k, 1 \leq x_{i} \leq t$, where $W_{x_{i}}$ is given as a class of the equitable refinement described above. More formally, let $\left(V_{1}, \ldots, V_{k}\right)$ be the $k$-partition of $\mathcal{H}$, and let $W_{x_{i}}, 1 \leq i \leq k$, $1 \leq x_{i} \leq t$ denote the vertex classes of the equitable refinement of $V=V_{1} \cup \ldots \cup V_{k}$ described above associated with the fixed partition $\mathcal{P}_{0}$. For convenience, we use the following definition.

Definition 7.3.3. We call any set $B \subseteq \bigcup_{1 \leq i \leq k} \bigcup_{1 \leq x_{i} \leq t} x_{i}$ transversal if it satisfies the following conditions:

(i) $2 \leq|B| \leq k$.

(ii) For all $\left\{x_{i}, y_{j}\right\} \in[B]^{2}, i \neq j$.

We are now ready for the following definition. Note that in what follows, with the notation ${ }^{s} B,{ }^{s} b$ used, we think of the " $s$ " as standing for "small".

Definition 7.3.4. Let ${ }^{s} B$ be transversal, where $\left|{ }^{s} B\right|={ }^{s} b \leq k$. We call the ${ }^{s} b$-partite cylinder $\bigcup_{\left\{x_{i}, y_{j}\right\} \in\left[{ }^{s} B\right]^{2}} P_{\alpha_{x_{i} y_{j}}}^{x_{i} y_{j}}$ of $\mathcal{P}_{0}, 1 \leq \alpha_{x_{i} y_{j}} \leq l_{x_{i} y_{j}} \leq l$, the small ${ }^{s} B$-cylinder, or more generally, a small cylinder. In the frequently occurring case that ${ }^{s} b=3$, we refer to this cylinder as a small triad.

Note that a small cylinder is uniquely determined by the choice of the transversal set ${ }^{s} B$, and choice of integers $\alpha_{x_{i} y_{j}}, 1 \leq \alpha_{x_{i} y_{j}} \leq l_{x_{i} y_{j}}$ for all $\left\{x_{i}, y_{j}\right\} \in\left[{ }^{s} B\right]^{2}$. Thus, if a small cylinder is $k$-partite, we may write it as $C_{k}=C_{k}\left({ }^{s} B,\left(\alpha_{x_{i} y_{j}}\right)_{\left\{x_{i}, y_{j}\right\} \in\left[{ }^{s} B\right]^{2}}\right)=$ $\bigcup_{\left\{x_{i}, y_{j}\right\} \in\left[{ }^{s} B\right]^{2}} P_{\alpha_{x_{i} y_{j}}}^{x_{i} y_{j}}$, for the choice of the transversal set ${ }^{s} B,\left|{ }^{s} B\right|={ }^{s} b=k$, and choice of integers $\alpha_{x_{i} y_{j}}, 1 \leq \alpha_{x_{i} y_{j}} \leq l_{x_{i} y_{j}}$, for all $\left\{x_{i}, y_{j}\right\} \in\left[{ }^{s} B\right]^{2}$. If the small cylinder is 3 -partite, that is, a small triad, then we write the cylinder as $C_{3}=C_{3}\left(\left\{x_{h}, y_{i}, z_{j}\right\},\left(\alpha_{x_{h} y_{i}}, \alpha_{x_{h} z_{j}}, \alpha_{y_{i} z_{j}}\right)\right)=$ $P_{\alpha_{x_{h} y_{i}}}^{x_{h} y_{i}} \cup P_{\alpha_{x_{h} z_{j}}}^{x_{h} z_{j}} \cup P_{\alpha_{y_{i} z_{j}}}^{y_{i} z_{j}}$ where $\left\{x_{h}, y_{i}, z_{j}\right\}$ is transversal, $1 \leq \alpha_{x_{h} y_{i}} \leq l_{x_{h} y_{i}}, 1 \leq \alpha_{x_{h} z_{j}} \leq l_{x_{h} z_{j}}$, $1 \leq \alpha_{y_{i} z_{j}} \leq l_{y_{i} z_{j}}$.

In what follows, any $k$-partite small cylinder $C_{k}$ of $\mathcal{P}_{0}$ is of the form

$$
C_{k}=C_{k}\left({ }^{s} B,\left(\alpha_{x_{i} y_{j}}\right)_{\left\{x_{i}, y_{j}\right\} \in\left[{ }^{s} B\right]^{2}}\right)=\bigcup_{\left\{x_{i}, y_{j}\right\} \in\left[{ }^{s} B\right]^{2}} P_{\alpha_{x_{i} y_{j}}}^{x_{i} y_{j}},
$$

where ${ }^{s} B$ is a transversal set, $\left|{ }^{s} B\right|={ }^{s} b=k$, and $\alpha_{x_{i} y_{j}}$ are integers such that $1 \leq \alpha_{x_{i} y_{j}} \leq$ $l_{x_{i} y_{j}}$ for all $\left\{x_{i}, y_{j}\right\} \in\left[{ }^{s} B\right]^{2}$. Similarly, any small triad $C_{3}$ of $\mathcal{P}_{0}$ is of the form

$$
C_{3}=C_{3}\left(\left\{x_{h}, y_{i}, z_{j}\right\},\left(\alpha_{x_{h} y_{i}}, \alpha_{x_{h} z_{j}}, \alpha_{y_{i} z_{j}}\right)\right)=P_{\alpha_{x_{h} y_{i}}}^{x_{h} y_{i}} \cup P_{\alpha_{x_{h} z_{j}}}^{x_{h} z_{j}} \cup P_{\alpha_{y_{i} z_{j}}}^{y_{i} z_{j}},
$$

where $\left\{x_{h}, y_{i}, z_{j}\right\}$ is transversal, $1 \leq \alpha_{x_{h} y_{i}} \leq l_{x_{h} y_{i}}, 1 \leq \alpha_{x_{h} z_{j}} \leq l_{x_{h} z_{j}}, 1 \leq \alpha_{y_{i} z_{j}} \leq l_{y_{i} z_{j}}$.

\section{Defective and Perfect Cylinders}

Here we consider 2-defective cylinders and several kinds of 3-defective cylinders. We note here that defective cylinders are always small cylinders. We begin with the following definition. 
Definition 7.3.5. We say that the $k$-partite small cylinder $C_{k}=\bigcup_{\left\{x_{i}, y_{j}\right\} \in\left[{ }^{s} B\right]^{2}} P_{\alpha_{x_{i} y_{j}}}^{x_{i} y_{j}}$ is a 2-defective cylinder if for some $\left\{x_{i}, y_{j}\right\} \in\left[{ }^{s} B\right]^{2}$, the bipartite graph $P_{\alpha_{x_{i}} y_{j}}^{x_{i} y_{j}}$ is not a $\left(\lambda l, \epsilon_{2}, 2\right)$-cylinder.

For some of the definitions which follow, it is necessary to have the following notion.

Definition 7.3.6. Let $C_{3}$ be a small triad. We define the 3-partite 3-cylinder $\mathcal{H}_{C_{3}}^{Q}$ to be that subhypergraph of $\mathcal{H}^{Q}$ induced on the underlying triangles of $C_{3}$, that is,

$$
\mathcal{H}_{C_{3}}^{Q}=\mathcal{H}^{Q} \cap \mathcal{K}_{3}^{(2)}\left(C_{3}\right) .
$$

We now describe specific classes of small triads $C_{3}$ which are in some way "defective".

Definition 7.3.7. Let $C_{3}=P_{\alpha_{x_{h} y_{i}}}^{x_{h} y_{i}} \cup P_{\alpha_{x_{h} z_{j}}}^{x_{h} z_{j}} \cup P_{\alpha_{y_{i} z_{j}}}^{y_{i} z_{j}}$ be a small $\left(\lambda l, \epsilon_{2}, 3\right)$-cylinder of $\mathcal{P}_{0}$. We say that $C_{3}$ is a regular-defective triad provided it satisfies that $\mathcal{H}_{C_{3}}^{Q}$ is not $\left(\delta, r_{1}\right)$-fully regular with respect to $C_{3}$.

We easily extend the notion of regular-defective from small triads $C_{3}$ to $k$-partite small cylinders $C_{k}$.

Definition 7.3.8. Suppose that $C_{k}=\bigcup_{\left\{x_{i}, y_{j}\right\} \in\left[{ }^{s} B\right]^{2}} P_{\alpha_{x_{i} y_{j}}}^{x_{i} y_{j}}$ is a $\left(\lambda l, \epsilon_{2}, k\right)$-cylinder of $\mathcal{P}_{0}$. We say that $C_{k}$ is a regular-defective cylinder if one of the small triads $C_{3}$ of $C_{k}$ is a regular-defective triad, that is, for some $\left\{x_{h}, y_{i}, z_{j}\right\} \in\left[{ }^{s} B\right]^{3}, C_{3}=P_{\alpha_{x_{h}} y_{i}}^{x_{h} y_{i}} \cup P_{\alpha_{x_{h}} z_{j}}^{x_{h} z_{j}} \cup P_{\alpha_{y_{i}} z_{j}}^{y_{i} z_{j}}$ is a regular-defective triad.

In addition to the class of regular-defective triads $C_{3}$, we are also interested in the following class of small triads, also "defective" in some way, and disjoint from the class of regular-defective triads described above.

Definition 7.3.9. Let $C_{3}=P_{\alpha_{x_{h} y_{i}}}^{x_{h} y_{i}} \cup P_{\alpha_{x_{h} z_{j}}}^{x_{h} z_{j}} \cup P_{\alpha_{y_{i} z_{j}}}^{y_{i} z_{j}}$ be a small $\left(\lambda l, \epsilon_{2}, 3\right)$-cylinder of $\mathcal{P}_{0}$. We say that $C_{3}$ is a dense-defective triad provided

(i) $C_{3}$ is not regular-defective, but

(ii) $C_{3}$ satisfies that $d_{\mathcal{H}^{Q}}\left(C_{3}\right) \leq \alpha-3 \delta$.

We now easily extend the notion of dense-defective from small triads $C_{3}$ to $k$-partite small cylinders $C_{k}$.

Definition 7.3.10. Suppose that $C_{k}=\bigcup_{\left\{x_{i}, y_{j}\right\} \in\left[{ }^{s} B\right]^{2}} P_{\alpha_{x_{i} y_{j}}}^{x_{i} y_{j}}$ is a $\left(\lambda l, \epsilon_{2}, k\right)$-cylinder of $\mathcal{P}_{0}$. We say that $C_{k}$ is a dense-defective cylinder if

a. $C_{k}$ is not a regular-defective cylinder, but

b. one of the small triads $C_{3}$ of $C_{k}$ is dense-defective, that is, for some $\left\{x_{h}, y_{i}, z_{j}\right\} \in$ $\left.{ }^{s} B\right]^{3} C_{3}=P_{\alpha_{x_{h} y_{i}}}^{x_{h} y_{i}} \cup P_{\alpha_{x_{h}} z_{j}}^{x_{h} z_{j}} \cup P_{\alpha_{y_{i} z_{j}}}^{y_{i} z_{j}}$ is a dense-defective triad.

Putting together the definitions in Definition 7.3.8 and Definition 7.3.10, we define 3-defective cylinders.

Definition 7.3.11. Suppose $C_{k}=\bigcup_{\left\{x_{i}, y_{j}\right\} \in\left[{ }^{s} B\right]^{2}} P_{\alpha_{x_{i} y_{j}}}^{x_{i} y_{j}}$ is a $k$-partite small cylinder of $\mathcal{P}_{0}$. We call the $k$-partite small cylinder $C_{k}$ a 3 -defective cylinder if it is either a regulardefective cylinder or a dense-defective cylinder. 
We now come to two classes of cylinders with which the remainder of the paper is concerned.

Definition 7.3.12. Suppose $C_{k}=\bigcup_{\left\{x_{i}, y_{j}\right\} \in\left[{ }^{s} B\right]^{2}} P_{\alpha_{x_{i} y_{j}}}^{x_{i} y_{j}}$ is a $k$-partite small cylinder of $\mathcal{P}_{0}$. We call the $k$-partite small cylinder $C_{k}$ a defective-cylinder if it is either a 2-defective cylinder or a 3-defective cylinder.

Now that we have described many different ways in which a $k$-partite small cylinder or a small triad can be defective, we identify a class of $k$-partite small cylinders which are not defective in the ways we saw above.

Definition 7.3.13. Suppose $C_{k}=\bigcup_{\left\{x_{i}, y_{j}\right\} \in\left[{ }^{s} B\right]^{2}} P_{\alpha_{x_{i}} y_{j}}^{x_{i} y_{j}}$ is a $k$-partite small cylinder of $\mathcal{P}_{0}$. We call the $k$-partite small cylinder $C_{k}$ a perfect cylinder if it is not a defective cylinder.

Note that a perfect cylinder $C_{k}=\bigcup_{\left\{x_{i}, y_{j}\right\} \in\left[{ }^{s} B\right]^{2}} P_{\alpha_{x_{i} y_{j}}}^{x_{i} y_{j}}$ satisfies all of the following:

(i) $C_{k}$ is a $\left(\lambda l, \epsilon_{2}, k\right)$-cylinder.

(ii) All $\left(\begin{array}{l}k \\ 3\end{array}\right)$ small triads $C_{3}=P_{\alpha_{x_{h}} y_{i}}^{x_{h} y_{i}} \cup P_{\alpha_{x_{h}} z_{j}}^{x_{h} z_{j}} \cup P_{\alpha_{y_{i} z_{j}}}^{y_{i} z_{j}},\left\{x_{h}, y_{i}, z_{j}\right\} \in\left[{ }^{s} B\right]^{3}$, satisfy that $\mathcal{H}_{C_{3}}^{Q}=\mathcal{H}^{Q} \cap \mathcal{K}_{3}^{(2)}\left(C_{3}\right)$ is $\left(\alpha-2 \delta, \delta, r_{1}\right)$ - regular with respect to $C_{3}$.

In what remains, we heavily use the following notation to denote the classes of $k$-partite small cylinders and small triads that were described above. To denote the $k$-partite small cylinders $C_{k}$ described above, let

$$
\begin{aligned}
2 \mathcal{D}_{k} & =\left\{C_{k}: C_{k} \text { is a } 2 \text {-defective cylinder of } \mathcal{P}_{0}\right\} \\
\mathcal{R D}_{k} & =\left\{C_{k}: C_{k} \text { is a regular-defective cylinder of } \mathcal{P}_{0}\right\} \\
\mathcal{D D}_{k} & =\left\{C_{k}: C_{k} \text { is a dense-defective cylinder of } \mathcal{P}_{0}\right\} \\
3 \mathcal{D}_{k} & =\left\{C_{k}: C_{k} \text { is a } 3 \text {-defective cylinder of } \mathcal{P}_{0}\right\} \\
\mathcal{D}_{k} & =\left\{C_{k}: C_{k} \text { is a defective cylinder of } \mathcal{P}_{0}\right\} \\
\Pi_{k} & =\left\{C_{k}: C_{k} \text { is a perfect cylinder of } \mathcal{P}_{0}\right\}
\end{aligned}
$$

To denote the small triads $C_{3}$ described above, let

$$
\begin{aligned}
& \mathcal{R D}_{3}=\left\{C_{3}: C_{3} \text { is a regular-defective triad of } \mathcal{P}_{0}\right\}, \\
& \mathcal{D D}_{3}=\left\{C_{3}: C_{3} \text { is a dense-defective triad of } \mathcal{P}_{0}\right\} .
\end{aligned}
$$

Note that in the notation above, the following trivial identities hold

$$
\begin{aligned}
\mathcal{R D}_{k} \cup \mathcal{D D}_{k} & =3 \mathcal{D}_{k}, \\
2 \mathcal{D}_{k} \cup 3 \mathcal{D}_{k} & =\mathcal{D}_{k} .
\end{aligned}
$$

For convenience, for any family $\mathcal{A}$ given by (106)-(111), we set

$$
\mathcal{K}_{k}^{(2)}(\mathcal{A})=\bigcup_{C_{k} \in \mathcal{A}} \mathcal{K}_{k}^{(2)}\left(C_{k}\right)
$$

Similarly, for any family $A$ given by (112) or (113), we set

$$
\mathcal{K}_{3}^{(2)}(\mathcal{A})=\bigcup_{C_{3} \in \mathcal{A}} \mathcal{K}_{3}^{(2)}\left(C_{3}\right)
$$


Our goal is to provide an upper bound on the quantity

$$
\left|\mathcal{K}_{k}^{(2)}\left(\mathcal{D}_{k}\right) \cap \bigcup_{s=1}^{r_{k}} \mathcal{K}_{k}^{(2)}(Q(s))\right|
$$

This upper bound is crucial for the proof of Proposition 7.2.4. We state this upper bound as a lemma itself. This is Lemma 7.3.14 advertised earlier in this section.

Lemma 7.3.14.

$$
\left|\mathcal{K}_{k}^{(2)}\left(\mathcal{D}_{k}\right) \cap \bigcup_{s=1}^{r_{k}} \mathcal{K}_{k}^{(2)}(Q(s))\right|<6 \sqrt{\delta} k^{3} N^{k} / \lambda^{\left(\begin{array}{c}
k \\
2
\end{array}\right)} .
$$

As mentioned earlier, we defer the proof of Lemma 7.3.14 until after the end of the proof of Proposition 7.2.4. Therefore, in that vein, we proceed with the proof of Proposition 7.2.4. However, a fact which we need there is the following corollary to Lemma 7.3.14. In this corollary, we are able to estimate the total number of perfect cylinders $C_{k}$.

\section{Corollary 7.3.15.}

$$
\left|\Pi_{k}\right|>\left(\left|\bigcup_{s=1}^{r_{k}} \mathcal{K}_{k}^{(2)}(Q(s))\right|-6 \sqrt{\delta} k^{3} \frac{N^{k}}{\lambda^{\left(\begin{array}{c}
k \\
2
\end{array}\right)}}\right) \frac{(\lambda l)^{\left(\begin{array}{c}
k \\
2
\end{array}\right)}}{m^{k}\left(1+\theta_{\lambda l, k}\left(\epsilon_{2}\right)\right)} .
$$

\section{Proof of Corollary 7.3.15.}

We use the following 2 obvious facts

$$
\left|\mathcal{K}_{k}^{(2)}\left(\Pi_{k}\right)\right| \geq\left|\mathcal{K}_{k}^{(2)}\left(\Pi_{k}\right) \cap \bigcup_{s=1}^{r_{k}} \mathcal{K}_{k}^{(2)}(Q(s))\right|
$$

and

$$
\left|\mathcal{K}_{k}^{(2)}\left(\Pi_{k}\right) \cap \bigcup_{s=1}^{r_{k}} \mathcal{K}_{k}^{(2)}(Q(s))\right|=\left|\bigcup_{s=1}^{r_{k}} \mathcal{K}_{k}^{(2)}(Q(s))\right|-\left|\mathcal{K}_{k}^{(2)}\left(\mathcal{D}_{k}\right) \cap \bigcup_{s=1}^{r_{k}} \mathcal{K}_{k}^{(2)}(Q(s))\right| .
$$

Combining (117) and (118) yields

$$
\left|\mathcal{K}_{k}^{(2)}\left(\Pi_{k}\right)\right| \geq\left|\bigcup_{s=1}^{r_{k}} \mathcal{K}_{k}^{(2)}(Q(s))\right|-\left|\mathcal{K}_{k}^{(2)}\left(\mathcal{D}_{k}\right) \cap \bigcup_{s=1}^{r_{k}} \mathcal{K}_{k}^{(2)}(Q(s))\right|,
$$

which in tandem with Lemma 7.3.14 implies

$$
\sum_{C_{k} \in \Pi_{k}}\left|\mathcal{K}_{k}^{(2)}\left(C_{k}\right)\right|>\left|\bigcup_{s=1}^{r_{k}} \mathcal{K}_{k}^{(2)}(Q(s))\right|-6 \sqrt{\delta} k^{3} \frac{N^{k}}{\lambda^{\left(\begin{array}{c}
k \\
2
\end{array}\right)}} .
$$

By Fact 2.1.7, each perfect cylinder $C_{k} \in \Pi_{k}$ satisfies

$$
\left|\mathcal{K}_{k}^{(2)}\left(C_{k}\right)\right|<\left(1+\theta_{\lambda l, k}\left(\epsilon_{2}\right)\right) \frac{m^{k}}{(\lambda l)^{\left(\begin{array}{c}
k \\
2
\end{array}\right)}} .
$$

We thus infer from (119) and (120) that

$$
\left|\Pi_{k}\right|>\left(\left|\bigcup_{s=1}^{r_{k}} \mathcal{K}_{k}^{(2)}(Q(s))\right|-6 \sqrt{\delta} k^{3} \frac{N^{k}}{\lambda^{\left(\begin{array}{l}
k \\
2
\end{array}\right)}}\right)(\lambda l)^{\left(\begin{array}{l}
k \\
2
\end{array}\right)} / m^{k}\left(1+\theta_{\lambda l, k}\left(\epsilon_{2}\right)\right),
$$

and so Corollary 7.3.15 is proved. 


\subsection{The Proof of Proposition 7.2.4.}

With Lemma 7.3.14 stated, we return to our original goal; proving Proposition 7.2.4. Recall that we were given $\vec{Q}=(Q(s)), 1 \leq s \leq r_{k}$, where for all $s \in\left[r_{k}\right], Q(s)=$ $\bigcup_{1 \leq i<j \leq k} Q^{i j}(s)$, and for all $\{i, j\} \in[k]^{2}$, for all $s \in\left[r_{k}\right], Q^{i j}(s) \subseteq G^{i j}$. Moreover, $\vec{Q}$ satisfied

$$
\left|\bigcup_{s=1}^{r_{k}} \mathcal{K}_{k}^{(2)}(Q(s))\right|>\delta_{k}\left|\mathcal{K}_{k}^{(2)}(G)\right|
$$

Proposition 7.2.4 states that

$$
\left|\mathcal{K}_{k}^{(3)}(\mathcal{H}) \cap \bigcup_{s=1}^{r_{k}} \mathcal{K}_{k}^{(2)}(Q(s))\right|>\left(\alpha^{\left(\begin{array}{c}
k \\
3
\end{array}\right)}-2 \delta_{k}\right)\left|\bigcup_{s=1}^{r_{k}} \mathcal{K}_{k}^{(2)}(Q(s))\right| .
$$

Recall Lemma 7.3.14 states

$$
\left|\mathcal{K}_{k}^{(2)}\left(\mathcal{D}_{k}\right) \cap \bigcup_{s=1}^{r_{k}} \mathcal{K}_{k}^{(2)}(Q(s))\right|<6 \sqrt{\delta} k^{3} \frac{N^{k}}{\lambda^{\left(\begin{array}{c}
k \\
2
\end{array}\right)}},
$$

where we recall $\mathcal{D}_{k}$ is the set of all $k$-partite defective cylinders. Recall that a cylinder $C_{k}$ is a defective cylinder if it is either

(i) 2-defective (i.e. not a $\left(\lambda l, \epsilon_{2}, k\right)$-cylinder),

(ii) regular-defective but not 2-defective (i.e. one of its $\left(\begin{array}{l}k \\ 3\end{array}\right)$ triads $C_{3}$ gives rise to $\mathcal{H}_{C_{3}}^{Q}$ which is not $\left(\delta, r_{1}\right)$-fully regular with respect to $\left.C_{3}\right)$,

(iii) dense-defective but not regular-defective or 2-defective (i.e. one of its $\left(\begin{array}{l}k \\ 3\end{array}\right)$ triads $C_{3}$ has density $d_{\mathcal{H}^{Q}}\left(C_{3}\right)$ no more than $\left.\alpha-3 \delta\right)$.

We now turn our attention to $\mathcal{K}_{k}^{(2)}\left(\Pi_{k}\right) \cap \bigcup_{s=1}^{r_{k}} \mathcal{K}_{k}^{(2)}(Q(s))$, where we recall that $\Pi_{k}$ is the set of all $k$-partite perfect cylinders. Recall that a cylinder $C_{k}$ is a perfect cylinder if it is a $\left(\lambda l, \epsilon_{2}, k\right)$-cylinder, all of whose $\left(\begin{array}{l}k \\ 3\end{array}\right)$ triads $C_{3}$ give rise to $\mathcal{H}_{C_{3}}^{Q}$ which is $\left(\alpha-2 \delta, \delta, r_{1}\right)$ regular with respect to $C_{3}$.

We first note that as a consequence of Lemma 7.3.14, nearly all of the copies of $\bigcup_{s=1}^{r_{k}} \mathcal{K}_{k}^{(2)}(Q(s))$ belong to perfect cylinders $C_{k}$. Said differently, the quantity $\mid \mathcal{K}_{k}^{(2)}\left(\Pi_{k}\right) \cap$ $\bigcup_{s=1}^{r_{k}} \mathcal{K}_{k}^{(2)}(Q(s)) \mid$ is nearly identical to $\left|\bigcup_{s=1}^{r_{k}} \mathcal{K}_{k}^{(2)}(Q(s))\right|$. To see this, recall that the $r_{k}$-tuple $\vec{Q}$ satisfied

$$
\left|\bigcup_{s=1}^{r_{k}} \mathcal{K}_{k}^{(2)}(Q(s))\right|>\delta_{k}\left|\mathcal{K}_{k}^{(2)}(G)\right|
$$

Since $G$ is a $(\lambda, \epsilon, k)$-cylinder, Fact 2.1 .7 says that

$$
\left|\mathcal{K}_{k}^{(2)}(G)\right|>\left(1-\theta_{k, \lambda}(\epsilon)\right) \frac{N^{k}}{\lambda^{(}\left(\begin{array}{l}
k \\
2
\end{array}\right)}>\frac{1}{2} \frac{N^{k}}{\lambda^{\left(\begin{array}{c}
k \\
2
\end{array}\right)}},
$$

where the last inequality follows from the fact that $\epsilon$ satisfies (96). Thus, we may apply the bound in (123) to further bound the inequality in (122) to conclude

$$
\left|\bigcup_{s=1}^{r_{k}} \mathcal{K}_{k}^{(2)}(Q(s))\right|>\frac{\delta_{k}}{2} \frac{N^{k}}{\lambda^{\left(\begin{array}{c}
k \\
2
\end{array}\right)}}
$$


Now, it is easy to bound the fraction

$$
\frac{\left|\mathcal{K}_{k}^{(2)}\left(\Pi_{k}\right) \cap \bigcup_{s=1}^{r_{k}} \mathcal{K}_{k}^{(2)}(Q(s))\right|}{\left|\bigcup_{s=1}^{r_{k}} \mathcal{K}_{k}^{(2)}(Q(s))\right|} .
$$

It follows from Lemma 7.3.14 and (124) that

$$
\begin{aligned}
\frac{\left|\mathcal{K}_{k}^{(2)}\left(\Pi_{k}\right) \cap \bigcup_{s=1}^{r_{k}} \mathcal{K}_{k}^{(2)}(Q(s))\right|}{\left|\bigcup_{s=1}^{r_{k}} \mathcal{K}_{k}^{(2)}(Q(s))\right|} & =\frac{\left|\bigcup_{s=1}^{r_{k}} \mathcal{K}_{k}^{(2)}(Q(s))\right|-\left|\mathcal{K}_{k}^{(2)}\left(\mathcal{D}_{k}\right) \cap \bigcup_{s=1}^{r_{k}} \mathcal{K}_{k}^{(2)}(Q(s))\right|}{\left|\bigcup_{s=1}^{r_{k}} \mathcal{K}_{k}^{(2)}(Q(s))\right|} \\
& >1-\frac{6 \sqrt{\delta} k^{3} N^{k}}{\lambda^{\left(\begin{array}{c}
k \\
2
\end{array}\right)}\left|\bigcup_{s=1}^{r_{k}} \mathcal{K}_{k}^{(2)}(Q(s))\right|} \\
& >1-\frac{12 \sqrt{\delta} k^{3}}{\delta_{k}}
\end{aligned}
$$

Note that in (126), $\delta$ satisfies $\delta \ll \delta_{k}$. In terms of the proportion, nearly all of the elements of $\bigcup_{s=1}^{r_{k}} \mathcal{K}_{k}^{(2)}(Q(s))$ are elements of $\mathcal{K}_{k}^{(2)}\left(\Pi_{k}\right)$.

We now complete the proof of Proposition 7.2.4. We know that almost all of the copies of $K_{k}^{(2)}$ from $\bigcup_{s=1}^{r_{k}} \mathcal{K}_{k}^{(2)}(Q(s))$ belong to perfect cylinders $C_{k}$. For each perfect cylinder $C_{k}$, we want to apply the statement $D(k)$ to 3 -cylinder $\mathcal{H}^{Q} \cap \mathcal{K}_{3}^{(2)}\left(C_{k}\right)$ with underlying cylinder $C_{k}$ to conclude that there are a sufficient number of copies of $K_{k}^{(3)}$ contained in $\mathcal{H}^{Q} \cap \mathcal{K}_{3}^{(2)}\left(C_{k}\right)$. We then sum the number of such copies of $K_{k}^{(3)}$ over all $C_{k} \in \Pi_{k}$ (the number of which we know from Corollary 7.3.15).

Let us first recall the formulation of the statement $D(k)$ (with a change in notation).

For all positive $\tilde{\alpha}, \tilde{\beta}$, there exists $\tilde{\delta}=\delta_{D(k)}(\tilde{\alpha}, \tilde{\beta})>0$ so that for all $\tilde{\lambda} \geq \frac{1}{\tilde{\delta}}$, there exist positive constants $\tilde{r}=r_{D(k)}(\tilde{\alpha}, \tilde{\beta}, \tilde{\delta}, \tilde{\lambda}), \tilde{\epsilon}=\epsilon_{D(k)}(\tilde{\alpha}, \tilde{\beta}, \tilde{\delta}, \tilde{\lambda})$ so that the following holds: suppose

(i) $\tilde{\mathcal{H}}$ is a k-partite 3-cylinder with k-partition $\left(\tilde{V}_{1}, \ldots, \tilde{V}_{k}\right),\left|\tilde{V}_{1}\right|=\ldots=\left|\tilde{V}_{k}\right|=\tilde{N}$.

(ii) $\tilde{G}=\bigcup_{1 \leq i<j \leq k} \tilde{G}^{i j}$ is a $(\tilde{\lambda}, \tilde{\epsilon}, \tilde{k})$ cylinder underlying $\tilde{\mathcal{H}}$.

(iii) For all $B \in[k]^{3}, \tilde{\mathcal{H}}(B)$ is $(\tilde{\alpha}, \tilde{\delta}, \tilde{r})$-regular with respect to $\tilde{G}(B)$.

Then $\tilde{\mathcal{H}}$ satisfies

$$
\left|\mathcal{K}_{k}^{(3)}(\tilde{\mathcal{H}})\right| \geq(1-\tilde{\beta}) \frac{(\tilde{\alpha})^{\left(\begin{array}{c}
k \\
3
\end{array}\right)}}{(\tilde{\lambda})^{\left(\begin{array}{c}
k \\
2
\end{array}\right)}}(\tilde{N})^{k} .
$$

Fix $C_{k} \in \Pi_{k}$ with $k$-partition $\left(W_{x_{1}}, \ldots, W_{x_{k}}\right)$. By definition of $\Pi_{k}, C_{k}$ is a $\left(\lambda l, \epsilon_{2}, k\right)$ cylinder which has all $\left(\begin{array}{l}k \\ 3\end{array}\right)$ triads $C_{3}$ giving rise to $\mathcal{H}_{C_{3}}^{Q}$ which is $\left(\alpha-2 \delta, \delta, r_{1}\right)$-regular with respect to $C_{3}$. Since we apply the statement $D(k)$ to the fixed cylinder $C_{k}$ and the 3-cylinder $\mathcal{H}^{Q} \cap \mathcal{K}_{3}^{(2)}\left(C_{k}\right)$, we set

$$
\begin{gathered}
\left(\tilde{V}_{1}, \ldots, \tilde{V}_{k}\right)=\left(W_{x_{1}}, \ldots, W_{x_{k}}\right), \\
\tilde{N}=m, \\
\tilde{\mathcal{H}}=\mathcal{H}^{Q} \cap \mathcal{K}_{3}^{(2)}\left(C_{k}\right)
\end{gathered}
$$




$$
\tilde{G}=C_{k} .
$$

Moreover, we need to verify that all the relevant constants $\alpha, \delta, \lambda l, r_{1}$, and $\epsilon_{2}$ that have already been fixed satisfy $D(k)$. More precisely, set

$$
\begin{gathered}
\tilde{\alpha}=\alpha-2 \delta, \\
\tilde{\beta}=\delta_{k} .
\end{gathered}
$$

The statement $D(k)$ guarantees constant $\tilde{\delta}$ given by $\tilde{\delta}=\delta_{D(k)}\left(\alpha-2 \delta, \delta_{k}\right)$. We see from (79) that

$$
\delta \leq \delta_{D(k)}\left(\alpha / 2, \delta_{k}\right) \leq \delta_{D(k)}\left(\alpha-2 \delta, \delta_{k}\right)=\tilde{\delta}
$$

Therefore, our choice of $\delta$ verifies the statement $D(k)$ for the choices $\tilde{\alpha}=\alpha-2 \delta$ and $\tilde{\beta}=\delta_{k}$. Since $\tilde{G}$ is a $\left(\lambda l, \epsilon_{2}, k\right)$-cylinder, set

$$
\tilde{\lambda}=\lambda l \text {. }
$$

It follows from (77) and (83) that

$$
\frac{1}{\tilde{\delta}}=\frac{1}{\delta_{D(k)}\left(\alpha-2 \delta, \delta_{k}\right)} \leq \frac{1}{\delta_{D(k)}\left(\alpha / 2, \delta_{k}\right)} \leq\left\lceil\frac{1}{\delta}\right\rceil \leq \lambda l_{0} \leq \lambda l=\tilde{\lambda} .
$$

Thus, $\tilde{\lambda} \geq \frac{1}{\delta}$. The statement $D(k)$ guarantees constants

$$
\tilde{r}=r_{D(k)}\left(\alpha-2 \delta, \delta_{k}, \delta, \lambda l\right)
$$

and

$$
\tilde{\epsilon}=\epsilon_{D(k)}\left(\alpha-2 \delta, \delta_{k}, \delta, \lambda l\right)
$$

By (85), we have

$$
r_{1}=r(t, l)=\max \left\{2 r_{k} t^{3} l^{3}, r_{D(k)}\left(\alpha-2 \delta, \delta_{k}, \delta, \lambda l\right)\right\} .
$$

Thus $\left.r_{1} \geq r_{D(k)}\left(\alpha-2 \delta, \delta_{k}, \delta, \lambda l\right)\right\}=\tilde{r}$ is an appropriate choice for $D(k)$ given the parameters in (131), (132), (133). By our choice of $\epsilon_{2}$ in (86),

$$
\epsilon_{2}(l) \leq \epsilon_{D(k)}\left(\alpha-2 \delta, \delta_{k}, \delta, \lambda l\right)=\tilde{\epsilon} .
$$

Thus we have that $\epsilon_{2}$ is an appropriate choice for $D(k)$ given the parameters in (131), (132), (133).

With the parameters given in (131)-(137), apply $D(k)$ to the cylinder $\tilde{G}$ and 3-cylinder $\tilde{\mathcal{H}}$. By that application,

$$
\left|\mathcal{K}_{k}^{(3)}(\tilde{\mathcal{H}})\right| \geq\left(1-\delta_{k}\right) \frac{(\alpha-2 \delta)^{\left(\begin{array}{l}
k \\
3
\end{array}\right)}}{(\lambda l)^{\left(\begin{array}{c}
k \\
2
\end{array}\right)}} m^{k} .
$$

Since $\mathcal{K}_{k}^{(3)}(\tilde{\mathcal{H}})=\mathcal{K}_{k}^{(3)}\left(\mathcal{H}^{Q}\right) \cap \mathcal{K}_{k}^{(2)}\left(C_{k}\right)$, (138) may be reformulated as

$$
\left|\mathcal{K}_{k}^{(3)}\left(\mathcal{H}^{Q}\right) \cap \mathcal{K}_{k}^{(2)}\left(C_{k}\right)\right| \geq\left(1-\delta_{k}\right) \frac{(\alpha-2 \delta)^{\left(\begin{array}{c}
k \\
3
\end{array}\right)}}{(\lambda l)^{\left(\begin{array}{c}
k \\
2
\end{array}\right)}} m^{k} .
$$


We repeat the argument above for all $C_{k} \in \Pi_{k}$ using (139) and the lower bound in Corollary 7.3.15 that

$$
\left|\Pi_{k}\right|>\left(\left|\bigcup_{s=1}^{r_{k}} \mathcal{K}_{k}^{(2)}(Q(s))\right|-6 \sqrt{\delta} k^{3} \frac{N^{k}}{\lambda^{\left(\begin{array}{c}
k \\
2
\end{array}\right)}}\right) \frac{(\lambda l)^{\left(\begin{array}{l}
k \\
2
\end{array}\right)}}{m^{k}\left(1+\theta_{\lambda l, k}\left(\epsilon_{2}\right)\right)} .
$$

In particular, we obtain

$$
\begin{aligned}
\left|\mathcal{K}_{k}^{(3)}\left(\mathcal{H}^{Q}\right) \cap \mathcal{K}_{k}^{(2)}\left(\Pi_{k}\right)\right| & =\left|\mathcal{K}_{k}^{(3)}\left(\mathcal{H}^{Q}\right) \cap \bigcup_{C_{k} \in \Pi_{k}} \mathcal{K}_{k}^{(2)}\left(C_{k}\right)\right| \\
& =\sum_{C_{k} \in \Pi_{k}}\left|\mathcal{K}_{k}^{(3)}\left(\mathcal{H}^{Q}\right) \cap \mathcal{K}_{k}^{(2)}\left(C_{k}\right)\right| \\
& >\left(\left|\bigcup_{s=1}^{r_{k}} \mathcal{K}_{k}^{(2)}(Q(s))\right|-6 \sqrt{\delta} k^{3} \frac{N^{k}}{\lambda^{\left(\begin{array}{c}
k \\
2
\end{array}\right)}}\right) \frac{(\alpha-2 \delta)^{\left(\begin{array}{l}
k \\
3
\end{array}\right)}\left(1-\delta_{k}\right)}{1+\theta_{\lambda l, k}\left(\epsilon_{2}\right)}
\end{aligned}
$$

the right hand side of which is equal to

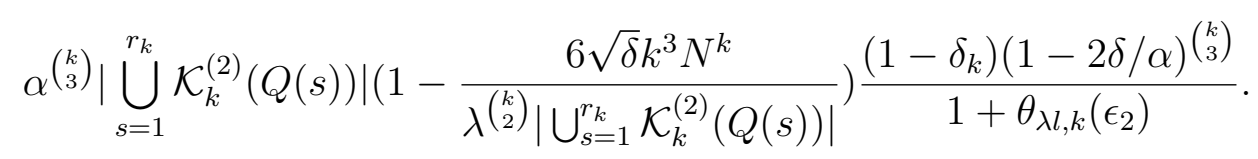

Using the fact that we chose $\epsilon_{2}$ to satisfy (89) and using (125) and (126), we see the above quantity is larger than

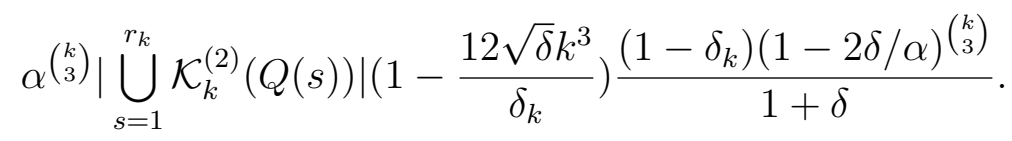

Since we chose $\delta$ to satisfy (78), we have that the above inequality is larger than

$$
\left(\alpha^{\left(\begin{array}{l}
k \\
3
\end{array}\right)}-2 \delta_{k}\right)\left|\bigcup_{s=1}^{r_{k}} \mathcal{K}_{k}^{(2)}(Q(s))\right| .
$$

Since $\left|\mathcal{K}_{k}^{(3)}(\mathcal{H}) \cap \bigcup_{s=1}^{r_{k}} \mathcal{K}_{k}^{(2)}(Q(s))\right| \geq\left|\mathcal{K}_{k}^{(3)}\left(\mathcal{H}^{Q}\right) \cap \mathcal{K}_{k}^{(2)}\left(\Pi_{k}\right)\right|$, Proposition 7.2.4 is proved by the inequalities above.

Now all that remains is to prove Lemma 7.3.14.

\subsection{Proof of Lemma 7.3.14.}

Recall that we are trying to show

$$
\left|\mathcal{K}_{k}^{(2)}\left(\mathcal{D}_{k}\right) \cap \bigcup_{s=1}^{r_{k}} \mathcal{K}_{k}^{(2)}(Q(s))\right|<6 \sqrt{\delta} k^{3} N^{k} / \lambda\left(\begin{array}{c}
k \\
2
\end{array}\right)
$$

For the proof of Lemma 7.3.14, we heavily use the following hierarchy already stated in (105).

$$
\alpha \gg \delta_{k} \gg \delta>\frac{1}{\lambda} \gg \epsilon_{1}, \frac{1}{(l \lambda)^{k}} \gg \epsilon_{2} \gg \frac{1}{T_{0}} \gg \epsilon \gg \frac{1}{N}
$$

The proof of Lemma 7.3.14 is easy once we have established some supplemental propositions. Indeed, in the upcoming Proposition 7.5.2, we show

$$
\left|\mathcal{K}_{k}^{(2)}\left(2 \mathcal{D}_{k}\right) \cap \bigcup_{s=1}^{r_{k}} \mathcal{K}_{k}^{(2)}(Q(s))\right|<\epsilon_{1} k^{2} N^{k}
$$


In the upcoming Propositions 7.5.3 and 7.5.4, we show

$$
\left|\mathcal{K}_{k}^{(2)}\left(\mathcal{R D}_{k}\right) \cap \bigcup_{s=1}^{r_{k}} \mathcal{K}_{k}^{(2)}(Q(s))\right|<3 \delta k^{3} \frac{N^{k}}{\lambda^{(k}\left(\begin{array}{l}
k \\
2
\end{array}\right)}
$$

and

$$
\left|\mathcal{K}_{k}^{(2)}\left(\mathcal{D D}_{k}\right) \cap \bigcup_{s=1}^{r_{k}} \mathcal{K}_{k}^{(2)}(Q(s))\right|<2 \sqrt{\delta} k^{3} \frac{N^{k}}{\lambda^{\left(\begin{array}{c}
k \\
2
\end{array}\right)}}
$$

Using the bounds in (142) and (143) and the easy identity $3 \mathcal{D}_{k}=\mathcal{R D}_{k} \cup \mathcal{D} \mathcal{D}_{k}$, we infer the inequality

$$
\left|\mathcal{K}_{k}^{(2)}\left(3 \mathcal{D}_{k}\right) \cap \bigcup_{s=1}^{r_{k}} \mathcal{K}_{k}^{(2)}(Q(s))\right|<5 \sqrt{\delta} k^{3} \frac{N^{k}}{\lambda^{\left(\begin{array}{c}
k \\
2
\end{array}\right)}}
$$

The inequality (140) easily follows from (141), (144) and (82) and the identity $\mathcal{D}_{k}=$ $2 \mathcal{D}_{k} \cup 3 \mathcal{D}_{k}$. This proves Lemma 7.3.14.

Thus, we need only prove Propositions 7.5.2, 7.5.3 and 7.5.4 to complete the proof of Lemma 7.3.14. We begin by proving Proposition 7.5.2, and need the following definition.

Definition 7.5.1. Let $x_{i}, y_{j}, \alpha_{x_{i} y_{j}}$, be such that $1 \leq i<j \leq k, 1 \leq x_{i}, y_{j} \leq t$, and $1 \leq \alpha_{x_{i} y_{j}} \leq l_{x_{i} y_{j}}$. An edge $e=\left\{v_{x_{i}}, v_{y_{j}}\right\} \in P_{\alpha_{x_{i} y_{j}}}^{x_{i} y_{j}}$ is called a bad edge if $P_{\alpha_{x_{i} y_{j}}}^{x_{i} y_{j}}$ is not a $\left(\lambda l, \epsilon_{2}, 2\right)$-cylinder.

Let $E_{\text {bad }} \subset G$ denote the set of all bad edges of $G$. Note that statement (ii) of Definition 6.1.2 implies

$$
\left|E_{b a d}\right| \leq \epsilon_{1} t^{2}\left(\begin{array}{l}
k \\
2
\end{array}\right) \frac{m^{2}}{\lambda}
$$

\section{Proposition 7.5.2.}

$$
\left|\mathcal{K}_{k}^{(2)}\left(2 \mathcal{D}_{k}\right) \cap \bigcup_{s=1}^{r_{k}} \mathcal{K}_{k}^{(2)}(Q(s))\right|<\epsilon_{1} k^{2} N^{k}
$$

\section{Proof of Proposition 7.5.2.}

It is a straightforward observation that

$$
\mathcal{K}_{k}^{(2)}\left(2 \mathcal{D}_{k}\right) \cap \bigcup_{s=1}^{r_{k}} \mathcal{K}_{k}^{(2)}(Q(s))=\left\{X \in \bigcup_{s=1}^{r_{k}} \mathcal{K}_{k}^{(2)}(Q(s)):[X]^{2} \cap E_{\text {bad }} \neq \emptyset\right\} .
$$

Let $e=\left\{v_{i}, v_{j}\right\} \in G, v_{i} \in V_{i}, v_{j} \in V_{j}, 1 \leq i<j \leq k$, be an arbitrary edge of $G$. Trivially,

$$
\left|\left\{X \in \mathcal{K}_{k}^{(2)}(G): v_{i}, v_{j} \in X\right\}\right| \leq N^{k-2}
$$

The fact that $m t=N,(145)$ and (147) combine to imply

therefore

$$
\left|\left\{X \in \mathcal{K}_{k}^{(2)}(G):[X]^{2} \cap E_{\text {bad }} \neq \emptyset\right\}\right|<\epsilon_{1}\left(\begin{array}{c}
k \\
2
\end{array}\right) \frac{N^{k}}{\lambda},
$$

$$
\left|\left\{X \in \bigcup_{s=1}^{r_{k}} \mathcal{K}_{k}^{(2)}(Q(s)):[X]^{2} \cap E_{b a d} \neq \emptyset\right\}\right|<\epsilon_{1}\left(\begin{array}{l}
k \\
2
\end{array}\right) \frac{N^{k}}{\lambda}<\epsilon_{1} k^{2} N^{k} .
$$


Proposition 7.5.2 then easily follows from our observation in (146).

\section{Proposition 7.5.3.}

$$
\left|\mathcal{K}_{k}^{(2)}\left(\mathcal{R D}_{k}\right) \cap \bigcup_{s=1}^{r_{k}} \mathcal{K}_{k}^{(2)}(Q(s))\right|<3 \delta k^{3} \frac{N^{k}}{\lambda^{\left(\begin{array}{l}
k \\
2
\end{array}\right)}}
$$

\section{Proof of Proposition 7.5.3.}

First, we estimate $\left|\mathcal{R} \mathcal{D}_{3}\right|$. From (71), we have that

$$
\sum\left\{\mu_{C_{3}}: C_{3} \text { is not a }\left(\delta, r_{1}\right) \text { - fully regular triad of } \mathcal{P}_{0}\right\}<\delta\left(\frac{k N}{m \lambda}\right)^{3}
$$

where we recall

$$
\mu_{C_{3}}=\frac{\left|\mathcal{K}_{3}^{(2)}\left(C_{3}\right)\right|}{m^{3}}
$$

It therefore follows that

$$
\sum_{C_{3} \in \mathcal{R} \mathcal{D}_{3}}\left|\mathcal{K}_{k}^{(2)}\left(C_{3}\right)\right|<\delta\left(\frac{k N}{\lambda}\right)^{3} .
$$

Since all regular-defective triads $C_{3}$ are $\left(\lambda l, \epsilon_{2}, 3\right)$-cylinders, we apply Fact 2.1.7 to (148) to obtain

$$
\left|\mathcal{R D}_{3}\right|\left(\frac{m}{\lambda l}\right)^{3}\left(1-\theta_{3, \lambda l}\left(\epsilon_{2}\right)\right)<\delta\left(\frac{k N}{\lambda}\right)^{3} .
$$

Since it follows from $(87)$ that $\theta_{3, \lambda l}\left(\epsilon_{2}\right)<1 / 2$, and given the fact that $m t=N$, we obtain from the above inequality

$$
\left|\mathcal{R} \mathcal{D}_{3}\right|<2 \delta k^{3} t^{3} l^{3}
$$

Now we estimate $\left|\mathcal{R} \mathcal{D}_{k}\right|$. For each regular-defective triad $C_{3}$, there are no more than

$$
t^{k-3} l^{\left(\begin{array}{c}
k \\
2
\end{array}\right)-3}
$$

regular-defective cylinders $C_{k}$ satisfying that $C_{3}$ is a triad of $C_{k}$. (149) and (150) combine to yield

$$
\left|\mathcal{R} \mathcal{D}_{k}\right|<2 \delta k^{3} t^{k} l\left(\begin{array}{l}
k \\
2
\end{array}\right)
$$

To complete the proof of Proposition 7.5.3, we need to estimate $\left|\mathcal{K}_{k}^{(2)}\left(\mathcal{R} \mathcal{D}_{k}\right)\right|$. Since each regular-defective cylinder $C_{k}$ is a $\left(\lambda l, \epsilon_{2}, k\right)$-cylinder, we apply Fact 2.1 .7 to each $C_{k} \in \mathcal{R} \mathcal{D}_{k}$ to obtain

$$
\left|\mathcal{K}_{k}^{(2)}\left(C_{k}\right)\right|<\left(1+\theta_{k, \lambda l}\left(\epsilon_{2}\right)\right) \frac{m^{k}}{(\lambda l)^{\left(\begin{array}{c}
k \\
2
\end{array}\right)}}
$$


Since we have in $(88)$ that $\theta_{k, \lambda l}\left(\epsilon_{2}\right)<1 / 2,(151)$ and (152) combine to yield

$$
\begin{aligned}
\left|\mathcal{K}_{k}^{(2)}\left(\mathcal{R D}_{k}\right)\right| & =\sum_{C_{k} \in \mathcal{R} \mathcal{D}_{k}}\left|\mathcal{K}_{k}^{(2)}\left(C_{k}\right)\right| \\
& <2 \delta k^{3} t^{k} l^{\left(\begin{array}{c}
k \\
2
\end{array}\right)}\left(1+\theta_{k, \lambda l}\left(\epsilon_{2}\right)\right) \frac{m^{k}}{(\lambda l)^{\left(\begin{array}{c}
k \\
2
\end{array}\right)}} \\
& <3 \delta k^{3} \frac{N^{k}}{\lambda^{\left(\begin{array}{c}
k \\
2
\end{array}\right)}} .
\end{aligned}
$$

Thus

$$
\left|\mathcal{K}_{k}^{(2)}\left(\mathcal{R} \mathcal{D}_{k}\right) \cap \bigcup_{s=1}^{r_{k}} \mathcal{K}_{k}^{(2)}(Q(s))\right| \leq\left|\mathcal{K}_{k}^{(2)}\left(\mathcal{R D}_{k}\right)\right|<3 \delta k^{3} \frac{N^{k}}{\lambda^{\left(\begin{array}{c}
k \\
2
\end{array}\right)}}
$$

which completes the proof of Proposition 7.5.3.

\section{Proposition 7.5.4.}

$$
\left|\mathcal{K}_{k}^{(2)}\left(\mathcal{D D}_{k}\right) \cap \bigcup_{s=1}^{r_{k}} \mathcal{K}_{k}^{(2)}(Q(s))\right|<2 \sqrt{\delta} k^{3} \frac{N^{k}}{\lambda^{\left(\begin{array}{c}
k \\
2
\end{array}\right)}}
$$

\section{Proof of Proposition 7.5.4.}

Proposition 7.5.4 follows from the following inequality:

$$
\left|\left\{X \in \bigcup_{s=1}^{r_{k}} \mathcal{K}_{k}^{(2)}(Q(s)):[X]^{3} \cap \mathcal{K}_{3}^{(2)}\left(\mathcal{D D}_{3}\right) \neq \emptyset\right\}\right|<2 \sqrt{\delta} k^{3} \frac{N^{k}}{\lambda^{\left(\begin{array}{c}
k \\
2
\end{array}\right)}} .
$$

Thus, it suffices to prove the inequality in (153) above. To that end, we begin by partitioning $\mathcal{D D}_{3}$ into two classes, $\mathcal{D} \mathcal{D}_{3}^{(1)}$ and $\mathcal{D} \mathcal{D}_{3}^{(2)}$, where

$$
\mathcal{D D}_{3}^{(1)}=\left\{C_{3} \in \mathcal{D D}_{3}:\left|\mathcal{K}_{3}^{(2)}\left(C_{3}\right) \cap \bigcup_{s=1}^{r_{k}} \mathcal{K}_{3}^{(2)}(Q(s))\right|<\sqrt{\delta}\left|\mathcal{K}_{3}^{(2)}\left(C_{3}\right)\right|\right\}
$$

and

$$
\mathcal{D D}_{3}^{(2)}=\mathcal{D D}_{3} \backslash \mathcal{D D}_{3}^{(1)}=\left\{C_{3} \in \mathcal{D D}_{3}:\left|\mathcal{K}_{3}^{(2)}\left(C_{3}\right) \cap \bigcup_{s=1}^{r_{k}} \mathcal{K}_{3}^{(2)}(Q(s))\right| \geq \sqrt{\delta}\left|\mathcal{K}_{3}^{(2)}\left(C_{3}\right)\right|\right\} .
$$

Set $\mathcal{K}_{3}^{(2)}\left(\mathcal{D D}_{3}^{(i)}\right)=\bigcup_{C_{3} \in \mathcal{D D}_{3}^{(i)}} \mathcal{K}_{3}^{(2)}\left(C_{3}\right), i=1,2$. To prove the inequality in (153), we show that

$$
\left|\left\{X \in \bigcup_{s=1}^{r_{k}} \mathcal{K}_{k}^{(2)}(Q(s)):[X]^{3} \cap \mathcal{K}_{3}^{(2)}\left(\mathcal{D D}_{3}^{(1)}\right) \neq \emptyset\right\}\right|<\sqrt{\delta} k^{3} \frac{N^{k}}{\lambda^{(k}\left(\begin{array}{l}
k \\
2
\end{array}\right)}
$$

and

$$
\left|\left\{X \in \bigcup_{s=1}^{r_{k}} \mathcal{K}_{k}^{(2)}(Q(s)):[X]^{3} \cap \mathcal{K}_{3}^{(2)}\left(\mathcal{D D}_{3}^{(2)}\right) \neq \emptyset\right\}\right|<\sqrt{\delta} k^{3} \frac{N^{k}}{\lambda^{\left(\begin{array}{c}
k \\
2
\end{array}\right)}} .
$$

To facilitate the proofs of the inequalities in (154) and (155), we define the following sets: for $B=\{a, b, c\} \in[k]^{3}$,

$$
\mathcal{D D}_{3}^{(1)}(B) \mathcal{D D}_{3}^{(1)}(B)=\left\{C_{3}=P_{\alpha_{x_{a} y_{b}}}^{x_{a} y_{b}} \cup P_{\alpha_{x_{a} z_{c}}}^{x_{a} z_{c}} \cup P_{\alpha_{y_{b} z_{c}}}^{y_{b} z_{c}} \in \mathcal{D D}_{3}^{(1)}\right\}
$$


and

$$
\mathcal{D D}_{3}^{(2)}(B) \mathcal{D D}_{3}^{(2)}(B)=\left\{C_{3}=P_{\alpha_{x_{a} y_{b}}}^{x_{a} y_{b}} \cup P_{\alpha_{x_{a} z_{c}}}^{x_{a} z_{c}} \cup P_{\alpha_{y_{b} z_{c}}}^{y_{b} z_{c}} \in \mathcal{D D}_{3}^{(2)}\right\}
$$

Thus, $\mathcal{D D}_{3}^{(1)}=\bigcup_{B \in[k]^{3}} \mathcal{D D}_{3}^{(1)}(B)$ and $\mathcal{D D}_{3}^{(2)}=\bigcup_{B \in[k]^{3}} \mathcal{D D}_{3}^{(2)}(B)$.

We begin our proof of (153) by establishing the inequality in (154). By definition of $\mathcal{D D}_{3}$, for each $C_{3} \in \mathcal{D D}_{3}^{(1)}, \mathcal{K}_{3}^{(2)}\left(C_{3}\right)$ intersects $\bigcup_{s=1}^{r_{k}} \mathcal{K}_{3}^{(2)}(Q(s))$ very little. The inequality in (154) is just the union of all these small intersections, and hence is small itself. We now show the details.

Fix $B \in[k]^{3}$, and let $C_{3} \in \mathcal{D D}_{3}^{(1)}(B)$. As a member of $\mathcal{D D}_{3}^{(1)}, C_{3}$ satisfies

$$
\left|\mathcal{K}_{3}^{(2)}\left(C_{3}\right) \cap \bigcup_{s=1}^{r_{k}} \mathcal{K}_{3}^{(2)}(Q(s))\right|<\sqrt{\delta}\left|\mathcal{K}_{3}^{(2)}\left(C_{3}\right)\right|
$$

Since $C_{3}$ is a $\left(\lambda l, \epsilon_{2}, 3\right)$-cylinder, we may apply Fact 2.1 .7 along with the inequality in (87) to further conclude

$$
\left|\mathcal{K}_{3}^{(2)}\left(C_{3}\right) \cap \bigcup_{s=1}^{r_{k}} \mathcal{K}_{3}^{(2)}(Q(s))\right|<\sqrt{\delta} \frac{m^{3}}{(\lambda l)^{3}}\left(1+\theta_{3, \lambda l}\left(\epsilon_{2}\right)\right)<2 \sqrt{\delta} \frac{m^{3}}{(\lambda l)^{3}} .
$$

Clearly, $\left|\mathcal{D D}_{3}^{(1)}(B)\right| \leq t^{3} l^{3}$, thus it follows from (156) that

$$
\left|\bigcup_{C_{3} \in \mathcal{D} \mathcal{D}_{3}(B)}\left(\mathcal{K}_{3}^{(2)}\left(C_{3}\right) \cap \bigcup_{s=1}^{r_{k}} \mathcal{K}_{3}^{(2)}(Q(s))\right)\right|<2 \sqrt{\delta} \frac{N^{3}}{\lambda^{3}} .
$$

Our next goal is to count the set of copies $X \in \mathcal{K}_{k}^{(2)}(G)$ such that $[X]^{3} \cap\left(\bigcup_{C_{3} \in \mathcal{D} \mathcal{D}_{3}^{(1)}(B)}\left(\mathcal{K}_{3}^{(2)}\left(C_{3}\right) \cap\right.\right.$ $\left.\left.\bigcup_{s=1}^{r_{k}} \mathcal{K}_{3}^{(2)}(Q(s))\right)\right) \neq \emptyset$. We first appeal to Fact 7.2.2. Since $G$ is a $(\lambda, \epsilon, k)$-cylinder, we may apply Fact 7.2.2 to conclude that all but $6 k \epsilon N^{3}$ triangles $\left\{v_{i_{1}}, v_{i_{2}}, v_{i_{3}}\right\} \in \mathcal{K}_{3}^{(2)}(G(B))$ satisfy that

$$
\left|\left\{X \in \mathcal{K}_{k}^{(2)}(G): v_{i_{1}}, v_{i_{2}}, v_{i_{3}} \in X\right\}\right|<\left(1+\theta_{k, \lambda}^{\prime \prime}(\epsilon)\right) \frac{N^{k-3}}{\lambda^{\left(\begin{array}{c}
k \\
2
\end{array}\right)-3}} .
$$

With $\epsilon$ satisfying (95), we conclude from (158) above that all but $6 k \epsilon N^{3}$ triangles $\left\{v_{i_{1}}, v_{i_{2}}, v_{i_{3}}\right\} \in \mathcal{K}_{3}^{(2)}(G(B))$ satisfy

$$
\left|\left\{X \in \mathcal{K}_{k}^{(2)}(G): v_{i_{1}}, v_{i_{2}}, v_{i_{3}} \in X\right\}\right|<2 \frac{N^{k-3}}{\lambda^{\left(\begin{array}{c}
k \\
2
\end{array}\right)-3}} .
$$

As a result of (157) and (159), we conclude that

$$
\begin{aligned}
\mid\left\{X \in \mathcal{K}_{k}^{(2)}(G):[X]^{3} \cap\right. & \left.\left(\bigcup_{C_{3} \in \mathcal{D} \mathcal{D}_{3}^{(1)}(B)}\left(\mathcal{K}_{3}^{(2)}\left(C_{3}\right) \cap \bigcup_{s=1}^{r_{k}} \mathcal{K}_{3}^{(2)}(Q(s))\right)\right) \neq \emptyset\right\} \mid \\
& <6 k \epsilon N^{k}+4 \sqrt{\delta} \frac{N^{k}}{\lambda^{\left(\begin{array}{c}
k \\
2
\end{array}\right)}}
\end{aligned}
$$


Summing (160) over all $\left(\begin{array}{l}k \\ 3\end{array}\right)$ sets $B \in[k]^{3}$, we easily infer from (160) that

$$
\left|\left\{X \in \bigcup_{s=1}^{r_{k}} \mathcal{K}_{k}^{(2)}(Q(s)):[X]^{3} \cap \mathcal{K}_{3}^{(2)}\left(\mathcal{D D}_{3}^{(1)}\right) \neq \emptyset\right\}\right|
$$

has upper bound

$$
6 k\left(\begin{array}{l}
k \\
3
\end{array}\right) \epsilon N^{k}+4 \sqrt{\delta}\left(\begin{array}{l}
k \\
3
\end{array}\right) \frac{N^{k}}{\lambda^{(}\left(\begin{array}{l}
k \\
2
\end{array}\right)}<5 \sqrt{\delta}\left(\begin{array}{l}
k \\
3
\end{array}\right) \frac{N^{k}}{\lambda^{(}\left(\begin{array}{l}
k \\
2
\end{array}\right)}
$$

where the last inequality follows from our choice of $\epsilon$ in (94). Thus, (154) is proved.

We now prove the inequality in (155). To that effect, for fixed $B \in[k]^{3}$, we show

$$
\left|\mathcal{D} \mathcal{D}_{3}^{(2)}(B)\right| \leq 2 \sqrt{\delta} t^{3} l^{3} .
$$

The inequality in (155) will easily follow from (161). Indeed, with (161) and each $C_{3} \in$ $\mathcal{D D}_{3}^{(2)}(B)$ by Fact 2.1 .7 satisfying $\left|\mathcal{K}_{3}^{(2)}\left(C_{3}\right)\right|<\frac{m^{3}}{(\lambda l)^{3}}\left(1+\theta_{3, \lambda l}\left(\epsilon_{2}\right)\right)$, we have

$$
\begin{aligned}
\left|\mathcal{K}_{3}^{(2)}\left(\mathcal{D D}_{3}^{(2)}(B)\right)\right| & =\left|\bigcup_{C_{3} \in \mathcal{D D}_{3}^{(2)}(B)} \mathcal{K}_{3}^{(2)}\left(C_{3}\right)\right|, \\
& <2 \sqrt{\delta} \frac{N^{3}}{\lambda^{3}}\left(1+\theta_{3, \lambda l}\left(\epsilon_{2}\right)\right), \\
& <3 \sqrt{\delta} \frac{N^{3}}{\lambda^{3}}
\end{aligned}
$$

where the last inequality follows from (87). Applying Fact 7.2.2 to $G(B)$ as before, we have

has upper bound

$$
\left|\left\{X \in \mathcal{K}_{k}^{(2)}(G):[X]^{3} \cap \mathcal{K}_{3}^{(2)}\left(\mathcal{D D}_{3}^{(2)}(B)\right) \neq \emptyset\right\}\right|
$$

$$
6 k \epsilon N^{k}+3 \sqrt{\delta} \frac{N^{k}}{\lambda^{\left(\begin{array}{c}
k \\
2
\end{array}\right)}}\left(1+\theta_{k, \lambda l}^{\prime \prime}(\epsilon)\right)<5 \sqrt{\delta} \frac{N^{k}}{\lambda^{\left(\begin{array}{c}
k \\
2
\end{array}\right)}}
$$

where the last inequality follows from (94). Thus, over all $B \in[k]^{3}$, we have

$$
\left|\left\{X \in \mathcal{K}_{k}^{(2)}(G):[X]^{3} \cap \mathcal{K}_{3}^{(2)}\left(\mathcal{D D}_{3}^{(2)}\right) \neq \emptyset\right\}\right|<5 \sqrt{\delta}\left(\begin{array}{l}
k \\
3
\end{array}\right) \frac{N^{k}}{\lambda^{\left(\begin{array}{l}
k \\
2
\end{array}\right)}},
$$

from which we infer

$$
\left|\left\{X \in \bigcup_{s=1}^{r_{k}} \mathcal{K}_{k}^{(2)}(Q(s)):[X]^{3} \cap \mathcal{K}_{3}^{(2)}\left(\mathcal{D D}_{3}^{(2)}\right) \neq \emptyset\right\}\right|<\sqrt{\delta} k^{3} \frac{N^{k}}{\lambda^{\left(\begin{array}{c}
k \\
2
\end{array}\right)}} .
$$

Thus, as promised, the inequality in (155) is established.

What remains to be shown is that for all $B \in[k]^{3},(161)$ holds. On the contrary, assume that there exists $B \in[k]^{3}$ such that

$$
\left|\mathcal{D} \mathcal{D}_{3}^{(2)}(B)\right| \geq\left\lceil 2 \sqrt{\delta} t^{3} l^{3}\right\rceil
$$

Our goal is to show that the assumption in (162) leads to a contradiction with our hypothesis that $\mathcal{H}(B)$ is $(\alpha, \delta, r)$-regular with respect to $G(B)$. That is, we show that from the set $\mathcal{D D}_{3}^{(2)}(B)$ being large as in $(162)$, we can construct a "witness against the regularity of $\mathcal{H}(B)$ with respect to $G(B)$ ". 
For convenience of notation in what follows, set

$$
\rho=\left\lceil 2 \sqrt{\delta} t^{3} l^{3}\right\rceil
$$

Recall that each $C_{3} \in \mathcal{D D}_{3}^{(2)}(B)$ satisfies

$$
\begin{aligned}
\left|\mathcal{K}_{3}^{(2)}\left(C_{3}\right) \cap \bigcup_{s=1}^{r_{k}} \mathcal{K}_{3}^{(2)}(Q(s))\right| & \geq \sqrt{\delta}\left|\mathcal{K}_{3}^{(2)}\left(C_{3}\right)\right| \\
& >\sqrt{\delta} \frac{m^{3}}{(\lambda l)^{3}}\left(1-\theta_{3, \lambda l}\left(\epsilon_{2}\right)\right)
\end{aligned}
$$

where the last inequality follows from Fact 2.1.7. To construct the witness, consider the following. Fix any $C_{3} \in \mathcal{D D}_{3}^{(2)}(B)$. For each $s \in\left[r_{k}\right], C_{3} \cap Q(s)$ is a 3-partite subgraph of $C_{3}$. Over all $r_{k}$ of the subgraphs $C_{3} \cap Q(s)$, we have an $r_{k}$-tuple of 3-partite subgraphs of $C_{3}$. Collecting all these subgraphs of $C_{3}$ and then letting $C_{3}$ run over the entire set $\mathcal{D D}_{3}^{(2)}(B)$ of $\rho$ small triads, we have a $\rho r_{k}$-tuple of 3-partite subgraphs of $G(B)$. Thus, our witness is the $\rho r_{k}$-tuple $\left(C_{3} \cap Q(s) ; C_{3} \in \mathcal{D D}_{3}^{(2)}(B), s \in\left[r_{k}\right]\right)$. To be formal, we assign an arbitrary numbering to this $\rho r_{k}$-tuple. Let $\phi: \mathcal{D D}_{3}^{(2)}(B) \times\left[r_{k}\right] \longrightarrow\left[\rho r_{k}\right]$ be an arbitrary bijection. Consider the $\rho r_{k}$-tuple of 3-partite subgraphs of $G(B)$ given by

$$
\overrightarrow{Q_{B}^{\prime}}=\left(Q_{B}^{\prime}(z): 1 \leq z \leq \rho r_{k}\right)
$$

where for each $z \in\left[\rho r_{k}\right]$,

$$
Q_{B}^{\prime}(z)=C_{3} \cap Q(s)
$$

where $C_{3} \in \mathcal{D D}_{3}^{(2)}(B), s \in\left[r_{k}\right]$ and $\phi\left(\left(C_{3}, s\right)\right)=z$. Note that

$$
\mathcal{K}_{3}^{(2)}\left(Q_{B}^{\prime}(z)\right)=\mathcal{K}_{3}^{(2)}\left(C_{3}\right) \cap \mathcal{K}_{3}^{(2)}(Q(s))
$$

Due to the inequality in (163),

$$
\begin{aligned}
\left|\bigcup_{z=1}^{\rho r_{k}} \mathcal{K}_{3}^{(2)}\left(Q_{B}^{\prime}(z)\right)\right| & =\left|\bigcup_{C_{3} \in \mathcal{D D}_{3}^{(2)}(B)} \bigcup_{s=1}^{r_{k}}\left(\mathcal{K}_{3}^{(2)}\left(C_{3}\right) \cap \mathcal{K}_{3}^{(2)}(Q(s))\right)\right|, \\
& =\left|\bigcup_{C_{3} \in \mathcal{D D}_{3}^{(2)}(B)}\left(\mathcal{K}_{3}^{(2)}\left(C_{3}\right) \cap \bigcup_{s=1}^{r_{k}} \mathcal{K}_{3}^{(2)}(Q(s))\right)\right|, \\
& >\left\lceil 2 \sqrt{\delta} t^{3} l^{3}\right\rceil \sqrt{\delta} \frac{m^{3}}{(\lambda l)^{3}}\left(1-\theta_{3, \lambda l}\left(\epsilon_{2}\right)\right), \\
& \geq 2\left(1-\theta_{3, \lambda l}\left(\epsilon_{2}\right)\right) \delta \frac{N^{3}}{\lambda^{3}}
\end{aligned}
$$

Combining the inequality in (87) with Fact 2.1.7, (165) and (95), we conclude

$$
2\left(1-\theta_{3, \lambda l}\left(\epsilon_{2}\right)\right) \delta \frac{N^{3}}{\lambda^{3}}>\frac{18}{10} \delta \frac{N^{3}}{\lambda^{3}}>\left(1+\theta_{3, l}(\epsilon)\right) \delta \frac{N^{3}}{\lambda^{3}}>\delta\left|\mathcal{K}_{3}^{(2)}(G(B))\right| .
$$

We thus conclude

$$
\left|\bigcup_{z=1}^{\rho r_{k}} \mathcal{K}_{3}^{(2)}\left(Q_{B}^{\prime}(z)\right)\right|>\delta\left|\mathcal{K}_{3}^{(2)}(G(B))\right|
$$


Since $\rho r_{k}=\left\lceil 2 \sqrt{\delta} t^{3} l^{3}\right\rceil r_{k} \leq 2 r_{k} T_{0}^{3} L_{0}^{3} \leq r,($ cf., $(97))$, it follows from the $(\alpha, \delta, r)$-regularity of $\mathcal{H}(B)$ with respect to $G(B)$ that

or equivalently,

$$
\left|\mathcal{H} \cap \bigcup_{z=1}^{\rho r_{k}} \mathcal{K}_{3}^{(2)}\left(Q_{B}^{\prime}(z)\right)\right|>(\alpha-2 \delta)\left|\bigcup_{z=1}^{\rho r_{k}} \mathcal{K}_{3}^{(2)}\left(Q_{B}^{\prime}(z)\right)\right|
$$

$$
\frac{\left|\mathcal{H} \cap \bigcup_{z=1}^{\rho r_{k}} \mathcal{K}_{3}^{(2)}\left(Q_{B}^{\prime}(z)\right)\right|}{\left|\bigcup_{z=1}^{\rho r_{k}} \mathcal{K}_{3}^{(2)}\left(Q_{B}^{\prime}(z)\right)\right|}>\alpha-2 \delta .
$$

Note that by definition in (164),

$$
\begin{aligned}
\frac{\left|\mathcal{H} \cap \bigcup_{z=1}^{\rho r_{k}} \mathcal{K}_{3}^{(2)}\left(Q_{B}^{\prime}(z)\right)\right|}{\left|\bigcup_{z=1}^{\rho r_{k}} \mathcal{K}_{3}^{(2)}\left(Q_{B}^{\prime}(z)\right)\right|}=\frac{\left|\mathcal{H} \cap\left(\bigcup_{C_{3} \in \mathcal{D D}_{3}^{(2)}(B)}\left(\mathcal{K}_{3}^{(2)}\left(C_{3}\right) \cap \bigcup_{s=1}^{r_{k}} \mathcal{K}_{3}^{(2)}(Q(s))\right)\right)\right|}{\left|\bigcup_{C_{3} \in \mathcal{D D}_{3}^{(2)}(B)}\left(\mathcal{K}_{3}^{(2)}\left(C_{3}\right) \cap \bigcup_{s=1}^{r_{k}} \mathcal{K}_{3}^{(2)}(Q(s))\right)\right|} \\
=\frac{\left|\bigcup_{C_{3} \in \mathcal{D D}_{3}^{(2)}(B)}\left(\mathcal{H} \cap\left(\mathcal{K}_{3}^{(2)}\left(C_{3}\right) \cap \bigcup_{s=1}^{r_{k}} \mathcal{K}_{3}^{(2)}(Q(s))\right)\right)\right|}{\left|\bigcup_{C_{3} \in \mathcal{D D}_{3}^{(2)}(B)}\left(\mathcal{K}_{3}^{(2)}\left(C_{3}\right) \cap \bigcup_{s=1}^{r_{k}} \mathcal{K}_{3}^{(2)}(Q(s))\right)\right|}
\end{aligned}
$$

The crucial observation here is that with $\mathcal{H}^{Q}=\mathcal{H} \cap \bigcup_{s=1}^{r_{k}} \mathcal{K}_{3}^{(2)}(Q(s))$, it follows that for each $C_{3} \in \mathcal{D D}_{3}^{(2)}(B)$,

$$
\mathcal{H} \cap\left(\mathcal{K}_{3}^{(2)}\left(C_{3}\right) \cap \bigcup_{s=1}^{r_{k}} \mathcal{K}_{3}^{(2)}(Q(s))=\mathcal{H}^{Q} \cap\left(\mathcal{K}_{3}^{(2)}\left(C_{3}\right) \cap \bigcup_{s=1}^{r_{k}} \mathcal{K}_{3}^{(2)}(Q(s))\right.\right.
$$

holds. Thus, we may rewrite (166) as

$$
\frac{\left|\mathcal{H}^{Q} \cap\left(\bigcup_{C_{3} \in \mathcal{D} \mathcal{D}_{3}^{(2)}(B)}\left(\mathcal{K}_{3}^{(2)}\left(C_{3}\right) \cap \bigcup_{s=1}^{r_{k}} \mathcal{K}_{3}^{(2)}(Q(s))\right)\right)\right|}{\left|\bigcup_{C_{3} \in \mathcal{D} \mathcal{D}_{3}^{(2)}(B)}\left(\mathcal{K}_{3}^{(2)}\left(C_{3}\right) \cap \bigcup_{s=1}^{r_{k}} \mathcal{K}_{3}^{(2)}(Q(s))\right)\right|}>\alpha-2 \delta .
$$

On the other hand, the following fact (which we prove momentarily) leads to a contradiction with (167).

Fact 7.5.5. For each $C_{3} \in \mathcal{D D}_{3}^{(2)}(B)$,

$$
\frac{\left|\mathcal{H}^{Q} \cap\left(\mathcal{K}_{3}^{(2)}\left(C_{3}\right) \cap \bigcup_{s=1}^{r_{k}} \mathcal{K}_{3}^{(2)}(Q(s))\right)\right|}{\left|\mathcal{K}_{3}^{(2)}\left(C_{3}\right) \cap \bigcup_{s=1}^{r_{k}} \mathcal{K}_{3}^{(2)}(Q(s))\right|} \leq \alpha-2 \delta .
$$

We prove Fact 7.5.5 immediately after we produce the promised contradiction to (167).

Indeed, by Fact 7.5.5, for each $C_{3} \in \mathcal{D D}_{3}^{(2)}(B)$,

$$
\left|\mathcal{H}^{Q} \cap\left(\mathcal{K}_{3}^{(2)}\left(C_{3}\right) \cap \bigcup_{s=1}^{r_{k}} \mathcal{K}_{3}^{(2)}(Q(s))\right)\right| \leq(\alpha-2 \delta)\left|\mathcal{K}_{3}^{(2)}\left(C_{3}\right) \cap \bigcup_{s=1}^{r_{k}} \mathcal{K}_{3}^{(2)}(Q(s))\right| .
$$

Summing (168) over all $C_{3} \in \mathcal{D D}_{3}^{(2)}(B)$ yields

$$
\sum_{C_{3} \in \mathcal{D D}_{3}^{(2)}(B)}\left|\mathcal{H}^{Q} \cap\left(\mathcal{K}_{3}^{(2)}\left(C_{3}\right) \cap \bigcup_{s=1}^{r_{k}} \mathcal{K}_{3}^{(2)}(Q(s))\right)\right| \leq(\alpha-2 \delta) \sum_{C_{3} \in \mathcal{D D}_{3}^{(2)}(B)}\left|\mathcal{K}_{3}^{(2)}\left(C_{3}\right) \cap \bigcup_{s=1}^{r_{k}} \mathcal{K}_{3}^{(2)}(Q(s))\right| .
$$


Note that for each $C_{3}, C_{3}^{\prime} \in \mathcal{D D}_{3}^{(2)}(B), C_{3} \neq C_{3}^{\prime}, \mathcal{K}_{3}^{(2)}\left(C_{3}\right) \cap \mathcal{K}_{3}^{(2)}\left(C_{3}^{\prime}\right)=\emptyset$. That is to say, the sets of triangles $\mathcal{K}_{3}^{(2)}\left(C_{3}\right)$ over distinct $C_{3} \in \mathcal{D D}_{3}^{(2)}(B)$ are pairwise disjoint. Thus, it follows that

$\sum_{C_{3} \in \mathcal{D} \mathcal{D}_{3}^{(2)}(B)}\left|\mathcal{H}^{Q} \cap\left(\mathcal{K}_{3}^{(2)}\left(C_{3}\right) \cap \bigcup_{s=1}^{r_{k}} \mathcal{K}_{3}^{(2)}(Q(s))\right)\right|=\left|\bigcup_{C_{3} \in \mathcal{D} \mathcal{D}_{3}^{(2)}(B)} \mathcal{H}^{Q} \cap\left(\mathcal{K}_{3}^{(2)}\left(C_{3}\right) \cap \bigcup_{s=1}^{r_{k}} \mathcal{K}_{3}^{(2)}(Q(s))\right)\right|$, and

$$
\sum_{C_{3} \in \mathcal{D D}_{3}^{(2)}(B)}\left|\mathcal{K}_{3}^{(2)}\left(C_{3}\right) \cap \bigcup_{s=1}^{r_{k}} \mathcal{K}_{3}^{(2)}(Q(s))\right|=\left|\bigcup_{C_{3} \in \mathcal{D D}_{3}^{(2)}(B)} \mathcal{K}_{3}^{(2)}\left(C_{3}\right) \cap \bigcup_{s=1}^{r_{k}} \mathcal{K}_{3}^{(2)}(Q(s))\right| .
$$

Thus, (169) may be rewritten as

$$
\left|\bigcup_{C_{3} \in \mathcal{D D}_{3}^{(2)}(B)} \mathcal{H}^{Q} \cap\left(\mathcal{K}_{3}^{(2)}\left(C_{3}\right) \cap \bigcup_{s=1}^{r_{k}} \mathcal{K}_{3}^{(2)}(Q(s))\right)\right| \leq(\alpha-2 \delta)\left|\bigcup_{C_{3} \in \mathcal{D D}_{3}^{(2)}(B)} \mathcal{K}_{3}^{(2)}\left(C_{3}\right) \cap \bigcup_{s=1}^{r_{k}} \mathcal{K}_{3}^{(2)}(Q(s))\right|,
$$

or equivalently,

$$
\frac{\left|\mathcal{H}^{Q} \cap\left(\bigcup_{C_{3} \in \mathcal{D D}_{3}^{(2)}(B)}\left(\mathcal{K}_{3}^{(2)}\left(C_{3}\right) \cap \bigcup_{s=1}^{r_{k}} \mathcal{K}_{3}^{(2)}(Q(s))\right)\right)\right|}{\left|\bigcup_{C_{3} \in \mathcal{D D}_{3}^{(2)}(B)}\left(\mathcal{K}_{3}^{(2)}\left(C_{3}\right) \cap \bigcup_{s=1}^{r_{k}} \mathcal{K}_{3}^{(2)}(Q(s))\right)\right|} \leq \alpha-2 \delta
$$

which is a direct contradiction with (167). This contradiction confirms that the assumption we made in (162) was false, hence, our proof of (161) is complete.

Proof of Fact 7.5.5.

Indeed, fix $C_{3} \in \mathcal{D D}_{3}^{(2)}(B)$. Recall that as an element of $\mathcal{D D}_{3}, C_{3}$ satisfies that $\mathcal{H}_{C_{3}}^{Q}$ is $\left(\delta, r_{1}\right)$-fully regular with respect to $C_{3}$, but $d_{\mathcal{H}_{C_{3}}^{Q}}\left(C_{3}\right)=\frac{\left|\mathcal{H}_{C_{3}}^{Q} \cap \mathcal{K}_{3}^{(2)}\left(C_{3}\right)\right|}{\left|\mathcal{K}_{3}^{(2)}\left(C_{3}\right)\right|} \leq \alpha-3 \delta$. As an element of $\mathcal{D D}_{3}^{(2)}(B)$,

$$
\begin{aligned}
\left|\mathcal{K}_{3}^{(2)}\left(C_{3}\right) \cap \bigcup_{s=1}^{r_{k}} \mathcal{K}_{3}^{(2)}(Q(s))\right| & \geq \sqrt{\delta}\left|\mathcal{K}_{3}^{(2)}\left(C_{3}\right)\right|, \\
& >\delta\left|\mathcal{K}_{3}^{(2)}\left(C_{3}\right)\right| .
\end{aligned}
$$

Note that $r_{k} \leq r_{1}$ follows from (85). We submit to $\mathcal{H}_{C_{3}}^{Q}$ the "witness" $\left(C_{3} \cap Q(s) ; s \in\left[r_{k}\right]\right)$. We conclude from the $\left(\delta, r_{1}\right)$-full regularity of $\mathcal{H}_{C_{3}}^{Q}$ that

$$
\left|\frac{\left|\mathcal{H}_{C_{3}}^{Q} \cap\left(\mathcal{K}_{3}^{(2)}\left(C_{3}\right) \cap \bigcup_{s=1}^{r_{k}} \mathcal{K}_{3}^{(2)}(Q(s))\right)\right|}{\left|\mathcal{K}_{3}^{(2)}\left(C_{3}\right) \cap \bigcup_{s=1}^{r_{k}} \mathcal{K}_{3}^{(2)}(Q(s))\right|}-d_{\mathcal{H}_{C_{3}}^{Q}}\left(C_{3}\right)\right|<\delta
$$

which is equivalent to

$$
\left|\frac{\left|\mathcal{H}^{Q} \cap\left(\mathcal{K}_{3}^{(2)}\left(C_{3}\right) \cap \bigcup_{s=1}^{r_{k}} \mathcal{K}_{3}^{(2)}(Q(s))\right)\right|}{\left|\mathcal{K}_{3}^{(2)}\left(C_{3}\right) \cap \bigcup_{s=1}^{r_{k}} \mathcal{K}_{3}^{(2)}(Q(s))\right|}-d_{\mathcal{H}_{C_{3}}^{Q}}\left(C_{3}\right)\right|<\delta .
$$


Thus, with $d_{\mathcal{H}_{C_{3}}^{Q}}\left(C_{3}\right) \leq \alpha-3 \delta$, we conclude

$$
\frac{\left|\mathcal{H}^{Q} \cap\left(\mathcal{K}_{3}^{(2)}\left(C_{3}\right) \cap \bigcup_{s=1}^{r_{k}} \mathcal{K}_{3}^{(2)}(Q(s))\right)\right|}{\left|\mathcal{K}_{3}^{(2)}\left(C_{3}\right) \cap \bigcup_{s=1}^{r_{k}} \mathcal{K}_{3}^{(2)}(Q(s))\right|} \leq \alpha-2 \delta .
$$

\section{The Upper Bound of TheOrem 3.1.1}

We begin this section by reviewing Theorem 3.1.1.

\subsection{Review of Theorem 3.1.1.}

Let us recall the of our work in Theorem 3.1.1 (i.e. Setup 1).

\section{Setup:}

For a given integer $k$, set of nonnegative reals $\left\{\alpha_{B}: B \in[k]^{3}\right\}$, constants $\delta, \lambda, r$, and $\epsilon$, suppose triple system $\mathcal{H}$ and underlying graph $G$ satisfy the following:

(i) $\mathcal{H}$ is a $k$-partite 3 -cylinder with $k$-partition $\left(V_{1}, \ldots, V_{k}\right),\left|V_{1}\right|=\ldots=\left|V_{k}\right|=n$.

(ii) $G=\bigcup_{1 \leq i<j \leq k} G^{i j}$ is an underlying $(\lambda, \epsilon, k)$-cylinder.

(iii) For all $B \in[k]^{3}, \mathcal{H}(B)$ is $\left(\alpha_{B}, \delta, r\right)$-fully regular with respect to $G(B)$.

Recall the Statement of Theorem 3.1.1.

Theorem 8.1.1. For all integers $k \geq 4$, for all sets of nonnegative reals $\left\{\alpha_{B}: B \in[k]^{3}\right\}$, for all $\beta>0$, there exists $\delta>0$ such that for all integers $\lambda>\frac{1}{\delta}$, there exist $r$ and $\epsilon$ so that the following holds: whenever triple system $\mathcal{H}$ and underlying cylinder $G$ satisfy the conditions of the Setup with parameters $k,\left\{\alpha_{B}: B \in[k]^{3}\right\}, \delta, \lambda, r$ and $\epsilon$, then

$$
\frac{\Pi_{B \in[k]]^{3} \alpha_{B}}}{\lambda^{\left(\begin{array}{c}
k \\
2
\end{array}\right)}} n^{k}(1-\beta) \leq\left|\mathcal{K}_{k}^{(3)}(\mathcal{H})\right| \leq \frac{\prod_{B \in[k]^{3} \alpha_{B}}}{\left.\lambda^{(k} \begin{array}{c}
k \\
2
\end{array}\right)} n^{k}(1+\beta) .
$$

We proved the lower bound in (170) in Theorem 3.2.1. What remains to be shown for the proof of Theorem 8.1.1 is the upper bound of (170). We mentioned earlier that the upper bound in (170) follows from the lower bound in (170). For that purpose, we recall Theorem 3.2.1 as a summary of the lower bound in (170).

Theorem 8.1.2. For all integers $k \geq 4$, for all sets of nonnegative reals $\left\{\alpha_{B}: B \in[k]^{3}\right\}$, for all $\beta>0$, there exists $\delta>0$ such that for all integers $\lambda>\frac{1}{\delta}$, there exist $r$ and $\epsilon$ so that the following holds: whenever triple system $\mathcal{H}$ and underlying cylinder $G$ satisfy the conditions of the Setup with parameters $k,\left\{\alpha_{B}: B \in[k]^{3}\right\}, \delta, \lambda, r$ and $\epsilon$, then

$$
\left|\mathcal{K}_{k}^{(3)}(\mathcal{H})\right| \geq \frac{\Pi_{B \in[k]^{3} \alpha_{B}}}{\lambda^{\left(\begin{array}{c}
k \\
2
\end{array}\right)}} n^{k}(1-\beta) .
$$

To complete the proof of Theorem 8.1.1, it suffices to prove the following proposition, which establishes the upper bound in (170). 
Proposition 8.1.3. For all integers $k \geq 4$, for all sets of nonnegative reals $\left\{\alpha_{B}: B \in\right.$ $\left.[k]^{3}\right\}$, for all $\beta>0$, there exists $\delta>0$ such that for all integers $\lambda>\frac{1}{\delta}$, there exist $r$ and $\epsilon$ so that the following holds: whenever triple system $\mathcal{H}$ and underlying cylinder $G$ satisfy the conditions of the Setup with parameters $k,\left\{\alpha_{B}: B \in[k]^{3}\right\}, \delta, \lambda, r$ and $\epsilon$, then

$$
\left|\mathcal{K}_{k}^{(3)}(\mathcal{H})\right| \leq \frac{\Pi_{B \in[k]^{3} \alpha_{B}}}{\left.\lambda^{(k} \begin{array}{c}
k \\
2
\end{array}\right)} n^{k}(1+\beta) .
$$

Before we begin the proof of Proposition 8.1.3, we first sketch the easy idea behind it. For each $\mathcal{D} \subseteq[k]^{3}$, consider $k$-partite 3 -cylinder $\mathcal{H}_{\mathcal{D}}=\bigcup_{B \in[k]^{3}} \mathcal{H}_{\mathcal{D}}(B)$, where for each $B \in[k]^{3}$,

$$
\mathcal{H}_{\mathcal{D}}(B)= \begin{cases}\mathcal{H}(B) & \text { if } B \notin \mathcal{D} \\ \mathcal{K}_{3}^{(2)}(G(B)) \backslash \mathcal{H}(B) & \text { if } B \in \mathcal{D}\end{cases}
$$

Clearly, $\mathcal{H}=\mathcal{H}_{\emptyset}$. We apply Theorem 8.1.2 to each $\mathcal{H}_{\mathcal{D}}, \mathcal{D} \neq \emptyset$, to conclude a lower bound on $\left|\mathcal{K}_{k}^{(3)}\left(\mathcal{H}_{\mathcal{D}}\right)\right|$. Since $\bigcup_{\mathcal{D} \subseteq[k]^{3}} \mathcal{K}_{k}^{(3)}\left(\mathcal{H}_{\mathcal{D}}\right)=\mathcal{K}_{k}^{(2)}(G)$, and $\left|\mathcal{K}_{k}^{(2)}(G)\right| \sim \frac{n^{k}}{\lambda_{\lambda}^{(k)}\left(\begin{array}{l}2 \\ 2\end{array}\right)}$, we are able to infer an upper bound on $\left|\mathcal{K}_{k}^{(3)}(\mathcal{H})\right|$, and show it is given by (172).

\subsection{Proof of Proposition 8.1.3.}

We now give the definitions of the constants involved in Proposition 8.1.3.

\section{Definitions of the Constants:}

Let $k \geq 4$ be a given integer, let $\left\{\alpha_{B}: B \in[k]^{3}\right\}$ be a given set of nonnegative reals, and let $\beta>0$ be a given constant. Define auxiliary positive constants

$$
\begin{aligned}
\beta^{\prime} & =\beta^{\prime}\left(k,\left\{\alpha_{B}: B \in[k]^{3}\right\}, \beta\right), \\
\theta & =\theta\left(k,\left\{\alpha_{B}: B \in[k]^{3}\right\}, \beta\right)
\end{aligned}
$$

to satisfy

$$
\frac{\theta+\beta^{\prime}}{\Pi_{B \in[k]^{3} \alpha_{B}}}-\beta^{\prime} \leq \beta .
$$

We now produce the constant $\delta$ promised by Proposition 8.1.3. Given the set $\left\{\alpha_{B}\right.$ : $\left.B \in[k]^{3}\right\}$, define for every $\mathcal{D} \subseteq[k]^{3}$ auxiliary sets $\left\{\alpha_{B}^{(\mathcal{D})}: B \in[k]^{3}\right\}$ according to the following rule: for every $B \in[k]^{3}$,

$$
\alpha_{B}^{(\mathcal{D})}= \begin{cases}\alpha_{B} & \text { if } B \notin \mathcal{D} \\ 1-\alpha_{B} & \text { if } B \in \mathcal{D}\end{cases}
$$

For $k \geq 4$ given above, for $\mathcal{D} \subseteq[k]^{3}$ and set $\left\{\alpha_{B}^{(\mathcal{D})}: B \in[k]^{3}\right\}$, and constant $\beta^{\prime}$ in $(174)$ given above, let

$$
\delta_{\mathcal{D}}=\delta_{\mathcal{D}}\left(k,\left\{\alpha_{B}^{(\mathcal{D})}: B \in[k]^{3}\right\}, \beta^{\prime}\right)
$$

be that constant guaranteed by Theorem 8.1.2. Set

$$
\delta=\min \left\{\delta_{\mathcal{D}}: \mathcal{D} \subseteq[k]^{3}\right\} .
$$

This concludes our definition of the promised constant $\delta$. 
Let $\lambda>\frac{1}{\delta}$ be a given integer. For $\mathcal{D} \subseteq[k]^{3}$, let

$$
\begin{aligned}
& r_{\mathcal{D}}=r_{\mathcal{D}}\left(k,\left\{\alpha_{B}^{(\mathcal{D})}: B \in[k]^{3}\right\}, \beta^{\prime}, \lambda\right), \\
& \epsilon_{\mathcal{D}}=\epsilon_{\mathcal{D}}\left(k,\left\{\alpha_{B}^{(\mathcal{D})}: B \in[k]^{3}\right\}, \beta^{\prime}, \lambda\right)
\end{aligned}
$$

be those constants guaranteed to exist by Theorem 8.1.2. Set

$$
r=\max \left\{r_{\mathcal{D}}: \mathcal{D} \subseteq[k]^{3}\right\}
$$

We now determine the value $\epsilon$ guaranteed to exist by Proposition 8.1.3. For this, we need to recall Fact 2.1.7 of Section 2. Recall that Fact 2.1.7 states that for all integers $k$ and $\lambda$ and suitably small constants $\epsilon$, there exists a function $\theta_{k, \lambda}(\epsilon), \theta_{k, \lambda}(\epsilon) \rightarrow 0$ as $\epsilon \rightarrow 0$, which satisfies the conclusion of Fact 2.1.7. Therefore, for $k$ and $\lambda$ given above, let $\epsilon^{\prime}$ be a positive constant so that the function $\theta_{k, \lambda}\left(\epsilon^{\prime}\right)$ guaranteed to exist by Fact 2.1.7 satisfies

$$
\theta_{k, \lambda}\left(\epsilon^{\prime}\right) \leq \theta
$$

where $\theta$ is that constant given in (175). Set

$$
\epsilon=\min \left\{\epsilon^{\prime}, \min \left\{\epsilon_{\mathcal{D}}: \mathcal{D} \subseteq[k]^{3}\right\}\right\} .
$$

This concludes our definitions of the constants guaranteed by Proposition 8.1.3. We now proceed to the proof of Proposition 8.1.3.

\section{Proof of Proposition 8.1.3.}

Let $k \geq 4$ be a given integer, let $\left\{\alpha_{B}: B \in[k]^{3}\right\}$ be a set of nonnegative reals, and let $\beta>0$ be a given constant. Let $\delta>0$ be given in (178). Let $\lambda>\frac{1}{\delta}$ be a given integer, and let $r$ and $\epsilon$ be given in (179) and (181) respectively. Suppose $\mathcal{H}$ is a triple system and $G$ is an underlying graph which with the parameters $k,\left\{\alpha_{B}: B \in[k]^{3}\right\}, \delta, \lambda, r$ and $\epsilon$ satisfy the hypothesis of the Setup. Our goal is to show

$$
\left|\mathcal{K}_{k}^{(3)}(\mathcal{H})\right| \leq \frac{\Pi_{B \in[k]^{3} \alpha_{B}}}{\lambda^{\left(\begin{array}{c}
k \\
2
\end{array}\right)}} n^{k}(1+\beta) .
$$

Our strategy is to use the lower bound in (171) for each $k$-partite 3 -cylinder $\mathcal{H}_{\mathcal{D}}$ defined in (173), where $\mathcal{D} \subseteq[k]^{3}, \mathcal{D} \neq \emptyset$.

For $\mathcal{D} \subseteq[k]^{3}$ and $B \in[k]^{3}$, recall that in (177) of the Definitions of the Constants, we defined

$$
\alpha_{B}^{(\mathcal{D})}= \begin{cases}\alpha_{B} & \text { if } B \notin \mathcal{D} \\ 1-\alpha_{B} & \text { if } B \in \mathcal{D} .\end{cases}
$$

Note that if $\mathcal{D}=\emptyset, \alpha_{B}^{(\mathcal{D})}=\alpha_{B}$ for all $B \in[k]^{3}$, and if $\mathcal{D}=[k]^{3}, \alpha_{B}^{(\mathcal{D})}=1-\alpha_{B}$ for all $B \in[k]^{3}$. We also note the following easy fact about the numbers $\alpha_{B}^{(\mathcal{D})}$.

Fact 8.2.1.

$$
\sum_{\mathcal{D} \subseteq[k]^{3}} \Pi_{B \in[k]^{3}} \alpha_{B}^{(\mathcal{D})}=1
$$

\section{Proof of Fact 8.2.1.}

Since

$$
\sum_{\mathcal{D} \subseteq[k]^{3}} \Pi_{B \in[k]^{3}} \alpha_{B}^{(\mathcal{D})}=\Pi_{B \in[k]^{3}}\left(\alpha_{B}^{(\emptyset)}+\alpha_{B}^{\left([k]^{3}\right)}\right)
$$


where for all $B \in[k]^{3}, \alpha_{B}^{(\emptyset)}=\alpha_{B}$ and $\alpha_{B}^{\left([k]^{3}\right)}=1-\alpha_{B}$, we see that Fact 8.2 .1 follows.

We also note the following (deeper) observations:

(a) For each $\mathcal{D} \subseteq[k]^{3}$, the $k$-partite 3 -cylinder $\mathcal{H}_{\mathcal{D}}=\bigcup_{B \in[k]^{3}} \mathcal{H}_{\mathcal{D}}(B)$ has underlying $(\lambda, \epsilon, k)$-cylinder $G=\bigcup_{1 \leq i<j \leq k} G^{i j}$.

(b) For each $\mathcal{D} \subseteq[k]^{3}$ and each $B \in[k]^{3}$, the $\left(\alpha_{B}, \delta, r\right)$-full regularity of $\mathcal{H}(B)$ with respect to $G(B)$ implies that $\mathcal{H}_{\mathcal{D}}(B)$ is $\left(\alpha_{B}^{(\mathcal{D})}, \delta, r\right)$-fully regular. In particular, for all $\mathcal{D} \subseteq[k]^{3}, \mathcal{H}_{\mathcal{D}}$ and $G$ satisfy the conditions of the Setup with parameters $k$, $\left\{\alpha_{B}^{(\mathcal{D})}: B \in[k]^{3}\right\}, \delta, \lambda, r, \epsilon$.

(c) For each $\mathcal{D} \subseteq[k]^{3}$, by our choices of $\delta, r$ and $\epsilon$ in (178), (179) and (181) respectively, Theorem 8.1.2 applies to $\mathcal{H}_{\mathcal{D}}$ and $G$ with the parameters $k,\left\{\alpha_{B}^{(\mathcal{D})}: B \in\right.$ $\left.[k]^{3}\right\}, \beta^{\prime}, \delta, \lambda, r, \epsilon$. We therefore conclude that for each $\mathcal{D} \subseteq[k]^{3}$,

$$
\left|\mathcal{K}_{k}^{(3)}\left(\mathcal{H}_{\mathcal{D}}\right)\right| \geq \frac{\Pi_{B \in[k]^{3} \alpha_{B}^{(\mathcal{D})}}}{\lambda^{\left(\begin{array}{c}
k \\
2
\end{array}\right)}} n^{k}\left(1-\beta^{\prime}\right) .
$$

We now combine our observation in $(c)$ above with Fact 8.2.1. For $\mathcal{D}_{1}, \mathcal{D}_{2} \subseteq[k]^{3}, \mathcal{D}_{1} \neq \mathcal{D}_{2}$, $\mathcal{K}_{k}^{(3)}\left(\mathcal{H}_{\mathcal{D}_{1}}\right) \cap \mathcal{K}_{k}^{(3)}\left(\mathcal{H}_{\mathcal{D}_{2}}\right)=\emptyset$. Moreover, $\bigcup_{\mathcal{D} \subseteq[k]^{3}} \mathcal{K}_{k}^{(3)}\left(\mathcal{H}_{\mathcal{D}}\right)=\mathcal{K}_{k}^{(2)}(G)$. Therefore,

$$
\sum_{\mathcal{D} \subseteq[k]^{3}}\left|\mathcal{K}_{k}^{(3)}\left(\mathcal{H}_{\mathcal{D}}\right)\right|=\left|\mathcal{K}_{k}^{(2)}(G)\right|
$$

Since $G$ is a $(\lambda, \epsilon, k)$-cylinder, we apply Fact 2.1.7 to conclude

$$
\left|\mathcal{K}_{k}^{(2)}(G)\right| \leq \frac{n^{k}}{\lambda^{\left(\begin{array}{c}
k \\
2
\end{array}\right)}}\left(1+\theta_{k, \lambda}(\epsilon)\right) \leq \frac{n^{k}}{\lambda^{\left(\begin{array}{c}
k \\
2
\end{array}\right)}}(1+\theta)
$$

where the last inequality follows from our choice of $\epsilon$ in (181). Combining the inequalities in (182) and (184) in (183) yields

$$
\left|\mathcal{K}_{k}^{(3)}\left(\mathcal{H}_{\emptyset}\right)\right|+\sum_{\substack{\mathcal{D} \subseteq[k]^{3} \\
\mathcal{D} \neq \emptyset}} \frac{\prod_{B \in[k]^{3}} \alpha_{B}^{(\mathcal{D})}}{\lambda^{\left(\begin{array}{c}
k \\
2
\end{array}\right)}} n^{k}\left(1-\beta^{\prime}\right) \leq \frac{n^{k}}{\lambda^{\left(\begin{array}{l}
k \\
2
\end{array}\right)}}(1+\theta)
$$

or equivalently, with $\mathcal{H}=\mathcal{H}_{\emptyset}$,

$$
\left|\mathcal{K}_{k}^{(3)}(\mathcal{H})\right| \frac{\lambda^{\left(\begin{array}{c}
k \\
2
\end{array}\right)}}{n^{k}\left(1-\beta^{\prime}\right)} \leq \frac{1+\theta}{1-\beta^{\prime}}-\sum_{\substack{\mathcal{D} \subseteq[k]^{3} \\
\mathcal{D} \neq \emptyset}} \Pi_{B \in[k]^{3}} \alpha_{B}^{(\mathcal{D})} .
$$

Employing the equality in Fact 8.2.1 in (185) yields

$$
\begin{aligned}
\left|\mathcal{K}_{k}^{(3)}(\mathcal{H})\right| \frac{\lambda^{\left(\begin{array}{c}
k \\
2
\end{array}\right)}}{n^{k}\left(1-\beta^{\prime}\right)} & \leq \frac{1+\theta}{1-\beta^{\prime}}-1+\Pi_{B \in[k]^{3}} \alpha_{B}^{(\emptyset)} \\
& =\Pi_{B \in[k]^{3} \alpha_{B}}\left(1+\frac{\theta+\beta^{\prime}}{\left(1-\beta^{\prime}\right) \Pi_{B \in[k]^{3}} \alpha_{B}}\right)
\end{aligned}
$$


or equivalently,

$$
\left|\mathcal{K}_{k}^{(3)}(\mathcal{H})\right| \leq \frac{\Pi_{B \in[k]^{3} \alpha_{B}}}{\lambda^{\left(\begin{array}{c}
k \\
2
\end{array}\right)}} n^{k}\left(1-\beta^{\prime}+\frac{\theta+\beta^{\prime}}{\prod_{B \in[k]^{3} \alpha_{B}}}\right) .
$$

Since we chose constants $\beta^{\prime}$ and $\theta$ to satisfy the inequality in (176), we conclude from (186) that

$$
\left|\mathcal{K}_{k}^{(3)}(\mathcal{H})\right| \leq \frac{\Pi_{B \in[k]^{3} \alpha_{B}}}{\lambda^{\left(\begin{array}{c}
k \\
2
\end{array}\right)}} n^{k}(1+\beta)
$$

and hence our proof of Proposition 8.1.1 is complete.

\section{Concluding Remarks}

In this section, we make brief remarks concerning Theorem 3.1.1. To begin, we consider the following slight generalization of The Counting Lemma. To discuss this statement, we define an appropriate context.

\section{Setup:}

For a given integer $k \geq 3$, for a fixed triple system $\mathcal{J}_{0}$ on vertex set $[k]$, for given constants $\alpha, \delta, \lambda, r$ and $\epsilon$, suppose $\mathcal{H}$ is a triple system and $G$ is an underlying graph satisfying the following:

(i) $\mathcal{H}$ is a $k$-partite 3 -cylinder with $k$-partition $\left(V_{1}, \ldots, V_{k}\right),\left|V_{1}\right|=\ldots=\left|V_{k}\right|=m$.

(ii) $G$ is an underlying $(\lambda, \epsilon, k)$-cylinder of $\mathcal{H}$.

(iii) for all $B \in \mathcal{J}_{0}, \mathcal{H}(B)$ is $(\alpha, \delta, r)$-fully regular with respect to the triad $G(B)$ (cf., Definition 2.2.7).

Denote by $\left(\begin{array}{c}\mathcal{H} \\ \mathcal{J}_{0}\end{array}\right)$ the set of copies of the hypergraph $\mathcal{J}_{0}$ in the hypergraph $\mathcal{H}$. We define the following special set $\mathcal{S}_{\mathcal{J}_{0}}(\mathcal{H}) \subseteq\left(\begin{array}{l}\mathcal{H} \\ \mathcal{J}_{0}\end{array}\right)$ of copies of $\mathcal{J}_{0}$ in $\mathcal{H}$ as

$$
\mathcal{S}_{\mathcal{J}_{0}}(\mathcal{H})=\left\{\mathcal{J} \in\left(\begin{array}{c}
\mathcal{H} \\
\mathcal{J}_{0}
\end{array}\right):[V(\mathcal{J})]^{2} \subseteq G\right\}
$$

Then the following may be proved using Theorem 3.1.1.

Theorem 9.0.2. For all integers $k \geq 4$ and triple systems $\mathcal{J}_{0} \subseteq[k]^{3}$, for all $\alpha, \beta>0$, there exists a constant $\delta>0$ so that for all integers $\lambda \geq \frac{1}{\delta}$, there exists an integer $r$ and $\epsilon>0$ so that whenever triple system $\mathcal{H}$ and $G=\bigcup_{1 \leq i<j \leq k} G^{i j}$ satisfy the conditions of the Setup above with constants $k, \alpha, \delta, \lambda, r$ and $\epsilon$ and triple system $\mathcal{J}_{0}$, then

$$
\frac{\alpha^{\left|\mathcal{J}_{0}\right|}}{\lambda^{\left(\begin{array}{c}
k \\
2
\end{array}\right)}} m^{k}(1-\beta) \leq\left|\mathcal{S}_{\mathcal{J}_{0}}(\mathcal{H})\right| \leq \frac{\alpha^{\left|\mathcal{J}_{0}\right|}}{\lambda^{\left(\begin{array}{c}
k \\
2
\end{array}\right)}} m^{k}(1+\beta) .
$$

Note that when $\mathcal{J}_{0}=K_{k}^{(3)}$, Theorem 9.0.2 reduces to Theorem 3.1.1. An application of Theorem 9.0.2 appears in [3].

The following theorem in [7] was proved using the Hypergraph Regularity Lemma of [2] in tandem with Theorem 3.1.1. Let a set of triple systems $\left\{\mathcal{F}_{i}\right\}_{i \in I}$ be given and define $\mathcal{P}_{n}$ to be the set of all 3 -uniform hypergraphs on vertex set $[n]$ not containing any 
$\mathcal{F} \in\left\{\mathcal{F}_{i}\right\}_{i \in I}$ as a subhypergraph. Denote by $\operatorname{ex}\left(n,\left\{\mathcal{F}_{i}\right\}_{i \in I}\right)$ the maximum size of a triple system in $\mathcal{P}_{n}$. Then

$$
\left|\mathcal{P}_{n}\right|=2^{\operatorname{ex}\left(n,\left\{\mathcal{F}_{i}\right\}_{i \in I}\right)+o\left(n^{3}\right)} .
$$

Note that (187) extends the result of Theorem 1.1.4 for triple systems. A recent extension of (187) in [4] provides a similar identity for $\left|\mathcal{P}_{n}^{\prime}\right|$, where $\mathcal{P}_{n}^{\prime}$ is defined as the set of triple systems on vertex set $[n]$ containing no $\mathcal{F} \in\left\{\mathcal{F}_{i}\right\}_{i \in I}$ as an induced subhypergraph.

Finally, in the upcoming paper [9], V. Rödl and J. Skokan developed a method for counting copies of $K_{5}^{(4)}$, the hypergraph consisting of 5 points and 5 quadruples, in an appropriate but quite technical environment. We remark that their environment is a variation of Setup 1, the environment which hosted Theorem 3.1.1. An alternative (and hopefully simpler) method for proving Theorem 3.1.1 is currently being explored in [8]. 


\section{Graph Terminology}

$k$-partite cylinder, ......5

triad, ... 5

$B$-cylinder, ...5

density

$d_{G}(X, Y), \ldots 6$

regular,

$\epsilon$-regular ...6

$(\gamma, \delta, r)$-regular, $\ldots 15$

$(\gamma, \delta, r, \lambda, \epsilon)$-regular couple, $\ldots 16$

$(\lambda, \epsilon, k)$-cylinder, $\ldots 6$

big/small

big $B$-cylinder, ... 39

big cylinder, . . . 39

big triad, ... 39

small ${ }^{s} B$-cylinder, . . 39

small cylinder, ... 39

small triad, . . 39

bad edge, ...47

\section{Graph Notation}

$G(B), \ldots 5$

$\mathcal{K}_{j}^{(2)}(G), \ldots 7$

$N_{j}(v), \ldots 6$

$N_{G, j}(v), \ldots 7$

$N_{F, j}(v), \ldots 7$

$G^{i j}(x, G), \ldots 16$

$P_{\alpha_{x_{i} y_{j}}}^{x_{i} y_{j}}, \ldots 28$

$C_{k}=C_{k}\left({ }^{s} B,\left(\alpha_{x_{i} y_{j}}\right)_{\left\{x_{i}, y_{j}\right\} \in\left[{ }^{s} B\right]^{2}}\right), \ldots 39$

$C_{3}=C_{3}\left(\left\{x_{h}, y_{i}, z_{j}\right\},\left(\alpha_{x_{h} y_{i}}, \alpha_{x_{h} z_{j}}, \alpha_{y_{i} z_{j}}\right)\right)$, ... 39

$E_{b a d}, \ldots 47$

Hypergraph Terminology

$k$-partite 3-cylinder, ...7

B-3-cylinder, ...7

underlies, ...7

density

$$
d_{\mathcal{H}}(G(B)), \ldots 8
$$

$d_{\mathcal{H}}(\vec{Q}), \ldots 8$

regular,

$(\delta, r)$-regular, $\ldots 8$

$(\alpha, \delta, r)$-regular, $\ldots 8$

$(\delta, r)$-irregular, $\ldots 8$

$(\delta, r)$-fully regular, $\ldots 9$

$(\alpha, \delta, r)$-fully regular, $\ldots 9$

Setup environments,

Setup $1, \ldots 9$

Setup $2, \ldots 10$

Setup $2^{\prime}, \ldots 10$
Statements

$D(k), \ldots 11$

$R(k), \ldots 11$

$\{i, j\}$-link graph of $x, \ldots 16$

link graph of $x, \ldots 16$

good vertex, ...21

defective/perfect cylinders

2-defective cylinder, ... 40

regular-defective triad, . . 40

regular-defective cylinder, . . . 40

dense-defective triad, . . 40

dense-defective cylinder, . . 40

3 -defective cylinder, ...40

defective-cylinder, . . .41

perfect cylinder, ...41

\section{Hypergraph Notation}

$\left|\mathcal{K}_{j}^{(3)}(\mathcal{H})\right|, \ldots 8$

$L_{x}^{i j}, \ldots 16$

$L_{x}, \ldots 16$

$V_{1}^{\prime}, \ldots 21$

$\mathcal{H}_{C_{3}}^{Q}, \ldots 40$

defective/perfect cylinders

$2 \mathcal{D}_{k}, \ldots 41$

$\mathcal{R D}_{k}, \ldots 41$

$\mathcal{D D}_{k}, \ldots 41$

$3 \mathcal{D}_{k}, \ldots 41$

$\mathcal{D}_{k}, \ldots 41$

$\Pi_{k}, \ldots 41$

$\mathcal{R D}_{3}, \ldots 41$

$\mathcal{D D}_{3}, \ldots 41$

$\mathcal{K}_{k}^{(2)}(\mathcal{A}), \ldots 41$

$\mathcal{D D}_{3}^{(1)}, \ldots 49$

$\mathcal{D D}_{3}^{(2)}, \ldots 49$

$\mathcal{D D}_{3}^{(1)}(B), \ldots 49$

$\mathcal{D D}_{3}^{(2)}(B), \ldots 50$

$\mathcal{H}_{\mathcal{D}}(B), \ldots 56$

$\alpha_{B}^{(\mathcal{D})}, \ldots 56$

\section{Partitions}

$\left(V_{1}, \ldots, V_{k}\right), \ldots 5$

equitable refinement, ...28

$W_{0} \cup \bigcup_{1 \leq i \leq k} \bigcup_{1 \leq x_{i} \leq t} W_{x_{i}}, \ldots 28$

$\left(\lambda l, k t, \epsilon_{1}, \epsilon_{2}\right)$-partition, $\ldots 28$

$\mathcal{P}, \ldots 28$

partition $\mathcal{P}$ is $(\delta, r)$-regular, $\ldots 29$

Miscellaneous Notation

$X \times Y, \ldots 5$

$[X]^{l}, \ldots 5$ 
${ }^{s} B, \ldots 39$

${ }^{s} b, \ldots 39$

$\bigcup_{1 \leq i \leq k} \bigcup_{1 \leq x_{i} \leq t} x_{i}, \ldots 28$ 


\section{REFERENCES}

[1] P. Erdös, P. Frankl, V. Rödl, "The asymptotic number of graphs not containing a fixed subgraph and a problem for hypergraphs having no exponent", Graphs and Combinatorics 2, pp. 113-121, 1986.

[2] P. Frankl, V. Rödl, "Extremal problems on set systems", Random Structures \& Algorithms, 20, (2002), no. 2, 131-164.

[3] P. Haxell, B. Nagle, V. Rödl, "Integer and fractional packings in dense 3-uniform hypergraphs", to appear, Random Structures \& Algorithms.

[4] Y. Kohayakawa, B. Nagle, V. Rödl, "Hereditary properties of triple systems", to appear, Combinatorics, Probability and Computing.

[5] J. Komlós, M. Simonovits, "Szemerédi's Regularity Lemma and its applications in graph theory", Combinatorics, Paul Erdös is eighty, Vol. 2, pp. 295-352, 1996.

[6] B. Nagle, "Regularity properties for triple systems", PhD Thesis, Emory University, 1999.

[7] B. Nagle, V. Rödl, "The asymptotic number of triple systems not containing a fixed one", Discrete Mathematics, 235, 2001, 271-290.

[8] Y. Peng, V. Rödl, J. Skokan, "Counting small cliques in 3-uniform hypergraphs", submitted.

[9] V. Rödl, J. Skokan, "Uniformity of set systems", submitted.

[10] I. Z. Ruzsa, E. Szemerédi, "Triple systems with no six points carrying three triangles", Combinatorics, Vol. II. Colloq. Math. Soc. J. Bolyai 18 North-Holland, 1978, 939-945.

[11] E. Szemerédi, "Regular partitions of graphs", Proc. Colloq. Int. CNRS, pp. 399-401, 1976.

[12] E. Szemerédi, "On sets of integers containing no $k$ elements in arithmic progression", Acta Arithmetica, Vol. 27, pp. 199-245, 1975.

School of Mathematics, Georgia Institute of Technology, Atlanta, GA, 30332, USA E-mail address: nagle@math.gatech.edu

Emory University, Atlanta, GA, 30032, USA

E-mail address: rodl@mathcs. emory.edu 